\title{
Package 'lavaan'
}

\author{
March 14, 2023
}

Title Latent Variable Analysis

Version $0.6-15$

Description Fit a variety of latent variable models, including confirmatory factor analysis, structural equation modeling and latent growth curve models.

Depends $\mathrm{R}(>=3.4)$

Imports methods, stats4, stats, utils, graphics, MASS, mnormt, pbivnorm, numDeriv, quadprog

License GPL ( $>=2)$

LazyData yes

ByteCompile true

URL https://lavaan.ugent. be

\section{NeedsCompilation no}

Author Yves Rosseel [aut, cre] (<https://orcid.org/0000-0002-4129-4477>), Terrence D. Jorgensen [aut] (<https://orcid.org/0000-0001-5111-6773>), Nicholas Rockwood [aut] (<https://orcid.org/0000-0001-5931-183X>), Daniel Oberski [ctb], Jarrett Byrnes [ctb], Leonard Vanbrabant [ctb], Victoria Savalei [ctb], Ed Merkle [ctb], Michael Hallquist [ctb], Mijke Rhemtulla [ctb], Myrsini Katsikatsou [ctb], Mariska Barendse [ctb], Florian Scharf [ctb],

Han Du [ctb]

Maintainer Yves Rosseel <Yves. Rosseel@UGent . be>

Repository CRAN

Date/Publication 2023-03-14 18:50:02 UTC 


\section{R topics documented:}

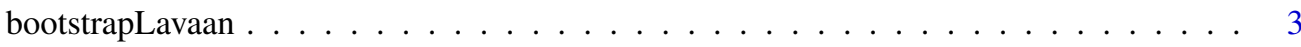

cfa .......................... 5

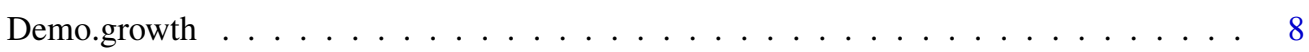

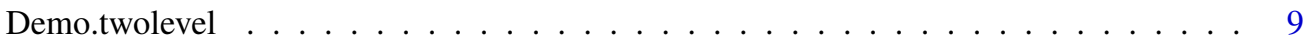

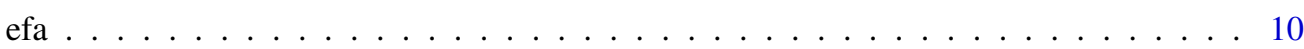

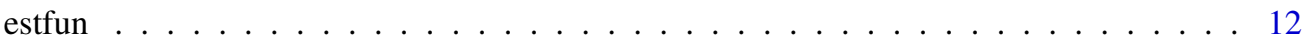

FacialBurns ........................... 13

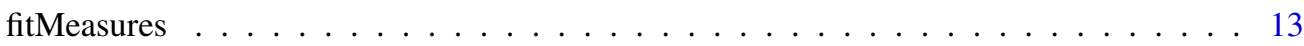

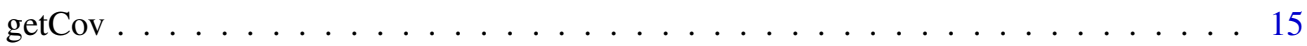

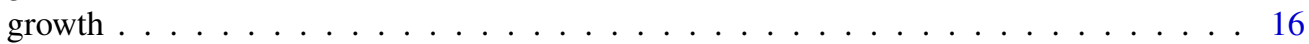

HolzingerSwineford1939 . . . . . . . . . . . . . . . . . 19

InformativeTesting . . . . . . . . . . . . . . . 20

InformativeTesting methods . . . . . . . . . . . . . . . 23

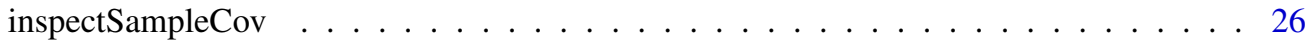

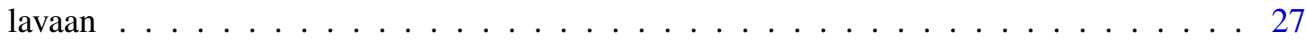

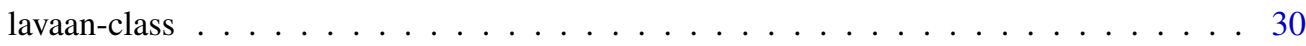

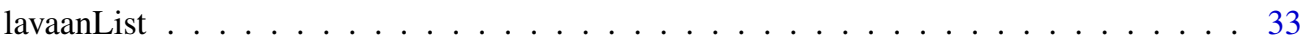

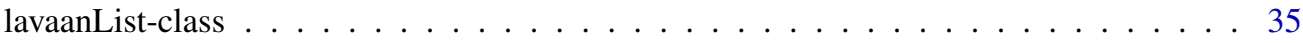

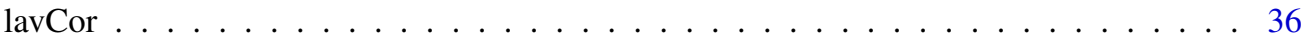

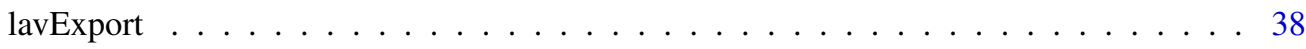

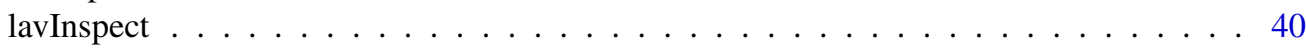

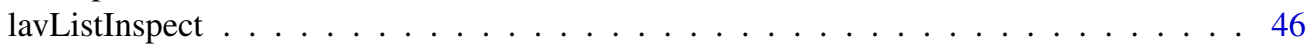

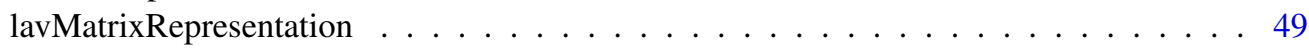

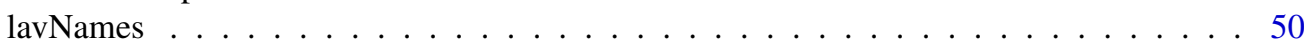

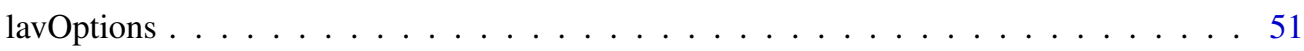

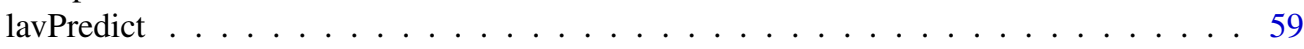

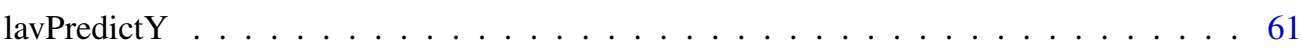

lavResiduals . . . . . . . . . . . . . . . . . . . 6 63

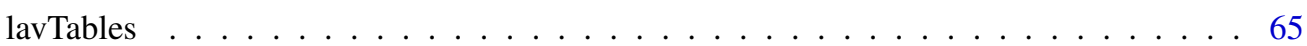

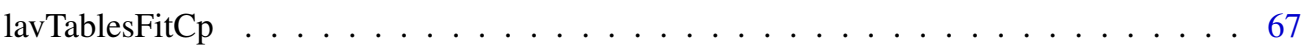

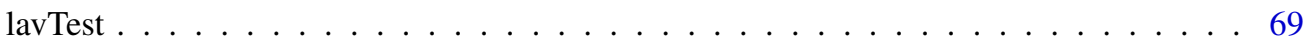

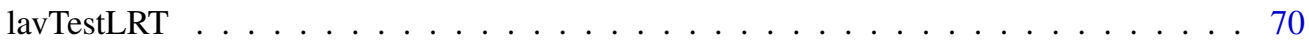

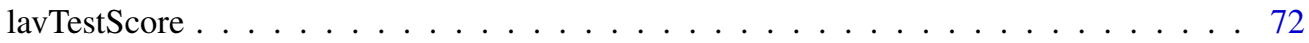

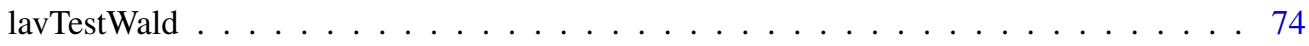

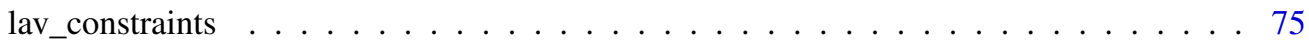

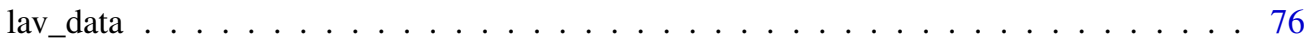

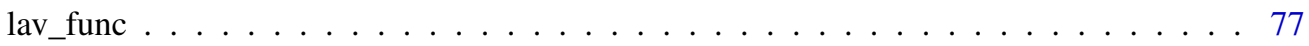

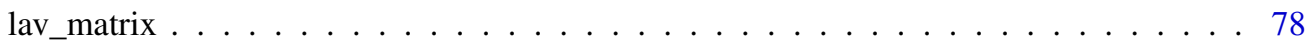

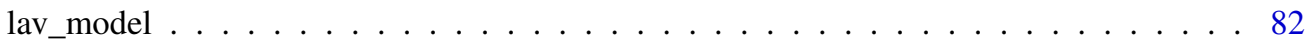

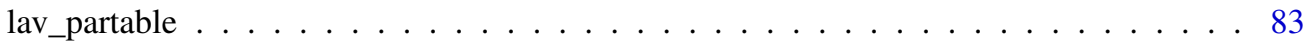

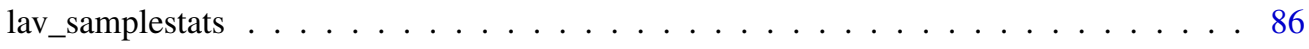

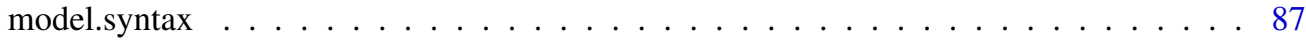

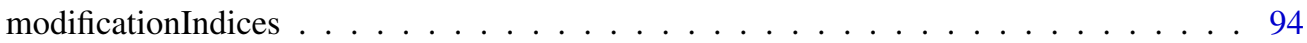

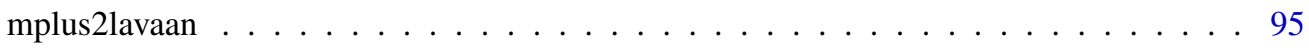

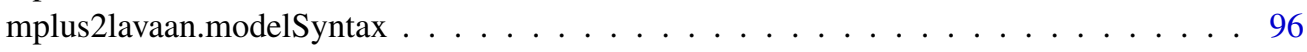

parameterEstimates . . . . . . . . . . . . . . . . . 97 


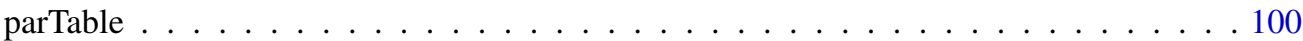

PoliticalDemocracy . . . . . . . . . . . . . . . . 100

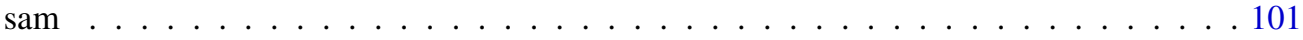

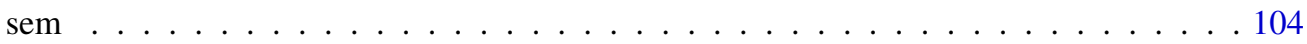

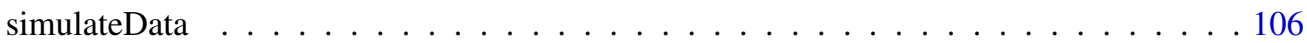

standardizedSolution . . . . . . . . . . . . . . . . . . . . . . . . . . . . . . . . . . . . . . . . . . . . . . . .

summary.efaList . . . . . . . . . . . . . . . . . . 111

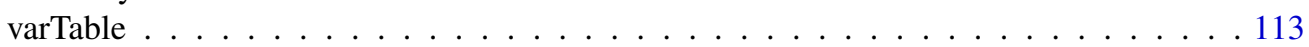

$\begin{array}{lr}\text { Index } & 114\end{array}$

bootstrapLavaan Bootstrapping a Lavaan Model

\section{Description}

Bootstrap the LRT, or any other statistic (or vector of statistics) you can extract from a fitted lavaan object.

Usage

bootstrapLavaan(object, $R=1000 \mathrm{~L}$, type = "ordinary", verbose = FALSE, FUN = "coef", keep.idx = FALSE, parallel = c("no", "multicore", "snow"), ncpus $=1 \mathrm{~L}, \mathrm{cl}=\mathrm{NULL}$, iseed $=$ NULL, h0.rmsea $=$ NULL, $\ldots$ )

bootstrapLRT(h0 = NULL, h1 = NULL, R = 1000L, type="bollen.stine", verbose = FALSE, return.LRT = FALSE, double. bootstrap = "no", double. bootstrap. $\mathrm{R}=500 \mathrm{~L}$, double. bootstrap.alpha $=0.05$, parallel = c("no", "multicore", "snow"), ncpus $=1 \mathrm{~L}, c l=N U L L$, iseed $=$ NULL)

\section{Arguments}

object An object of class lavaan.

ho

An object of class lavaan. The restricted model.

h1

An object of class lavaan. The unrestricted model.

$\mathrm{R} \quad$ Integer. The number of bootstrap draws.

type If "ordinary" or "nonparametric", the usual (naive) bootstrap method is used. If "bollen.stine", the data is first transformed such that the null hypothesis holds exactly in the resampling space. If "yuan", the data is first transformed by combining data and theory (model), such that the resampling space is closer to the population space. Note that both "bollen.stine" and "yuan" require the data to be continuous. They will not work with ordinal data. If "parametric", the parametric bootstrap approach is used; currently, this is only valid for continuous data following a multivariate normal distribution. See references for more details. 


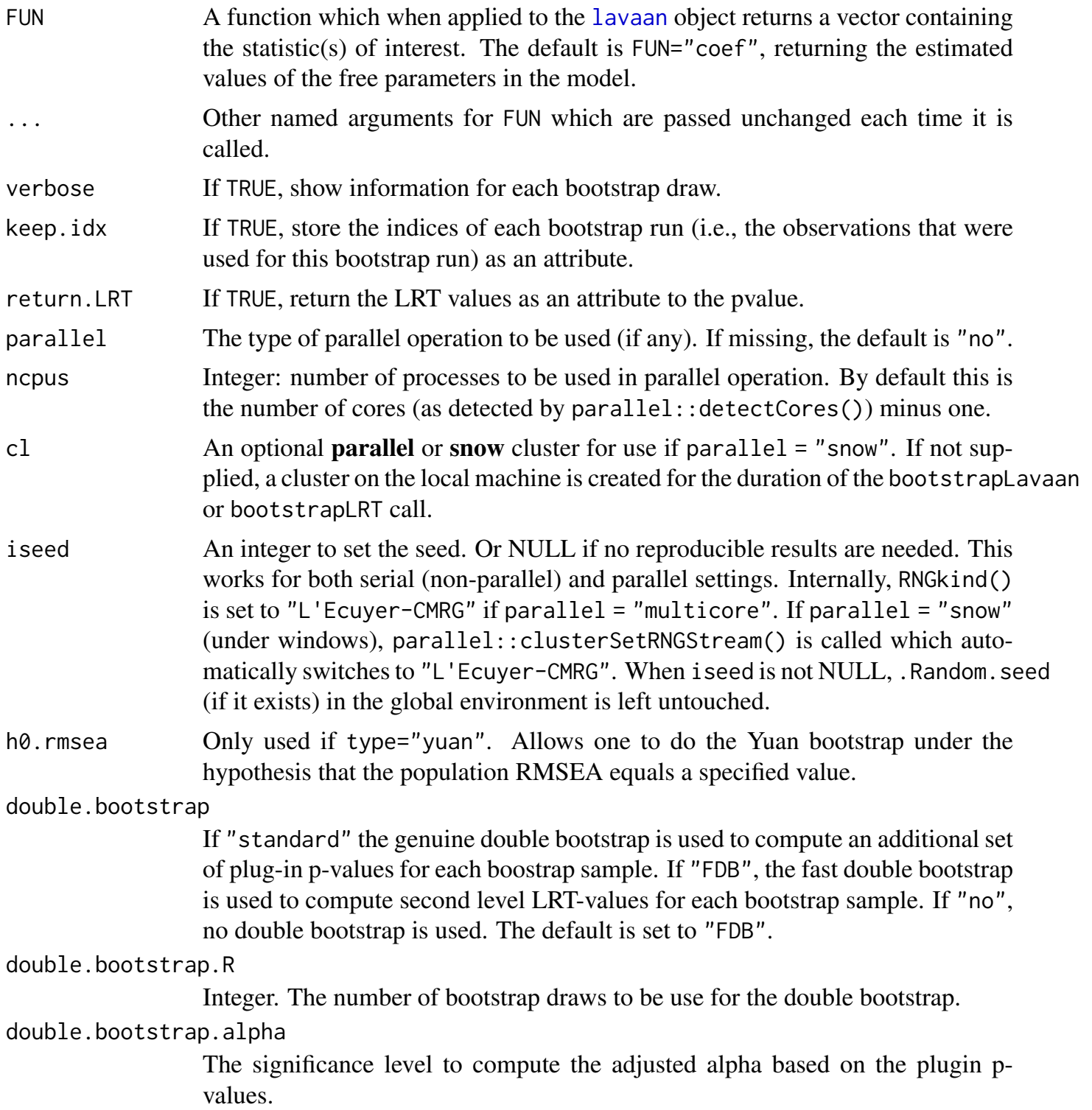

\section{Details}

The FUN function can return either a scalar or a numeric vector. This function can be an existing function (for example coef) or can be a custom defined function. For example:

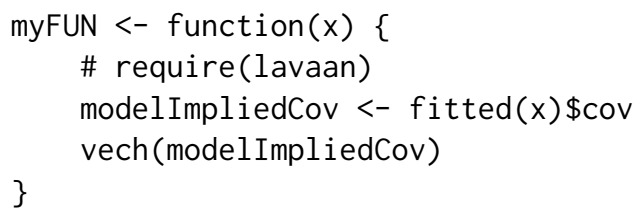

If parallel="snow", it is imperative that the require(lavaan) is included in the custom function. 
Value

For bootstrapLavaan(), the bootstrap distribution of the value(s) returned by FUN, when the object can be simplified to a vector. For bootstrapLRT(), a bootstrap $p$ value, calculated as the proportion of bootstrap samples with a LRT statistic at least as large as the LRT statistic for the original data.

\section{Author(s)}

Yves Rosseel and Leonard Vanbrabant. Ed Merkle contributed Yuan's bootstrap. Improvements to Yuan's bootstrap were contributed by Hao Wu and Chuchu Cheng. The handling of iseed was contributed by Shu Fai Cheung.

\section{References}

Bollen, K. and Stine, R. (1992) Bootstrapping Goodness of Fit Measures in Structural Equation Models. Sociological Methods and Research, 21, 205-229.

Yuan, K.-H., Hayashi, K., \& Yanagihara, H. (2007). A class of population covariance matrices in the bootstrap approach to covariance structure analysis. Multivariate Behavioral Research, 42, 261-281.

\section{Examples}

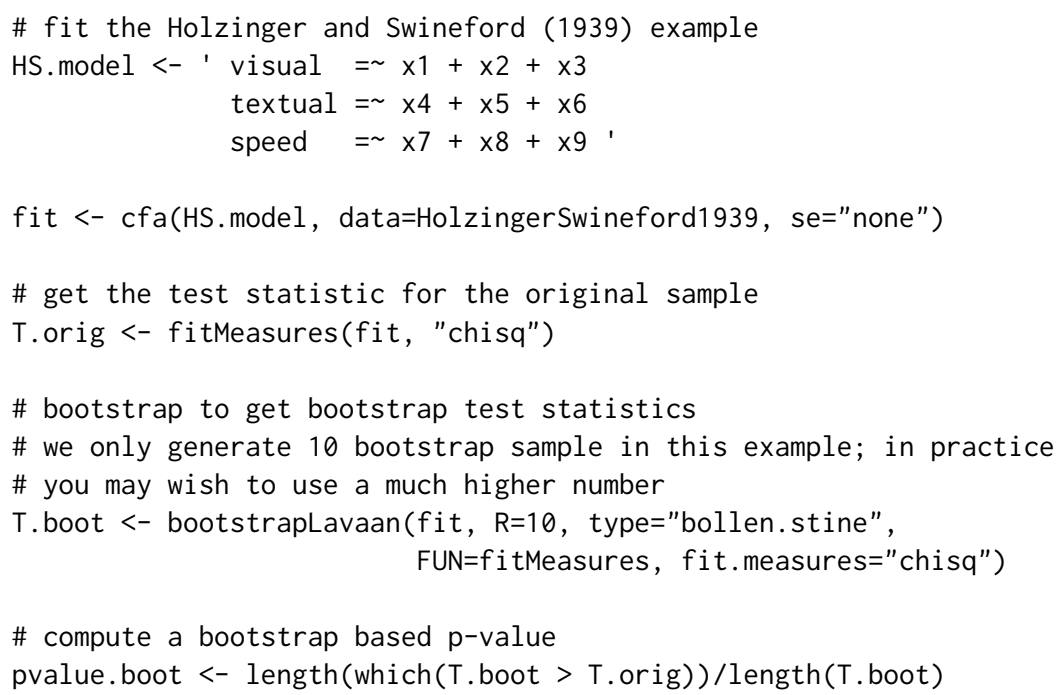

cfa

Fit Confirmatory Factor Analysis Models

\section{Description}

Fit a Confirmatory Factor Analysis (CFA) model. 


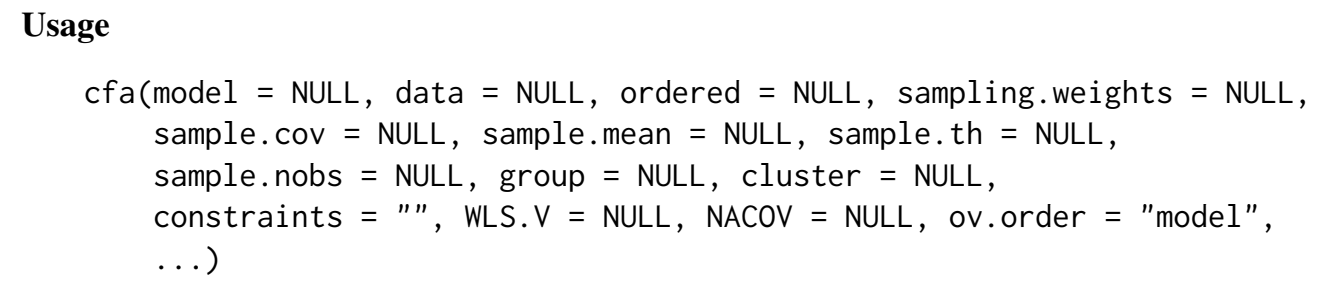

\section{Arguments}

model

A description of the user-specified model. Typically, the model is described using the lavaan model syntax. See model . syntax for more information. Alternatively, a parameter table (eg. the output of the lavaanify() function) is also accepted.

data An optional data frame containing the observed variables used in the model. If some variables are declared as ordered factors, lavaan will treat them as ordinal variables.

ordered Character vector. Only used if the data is in a data.frame. Treat these variables as ordered (ordinal) variables, if they are endogenous in the model. Importantly, all other variables will be treated as numeric (unless they are declared as ordered in the data.frame.) Since 0.6-4, ordered can also be logical. If TRUE, all observed endogenous variables are treated as ordered (ordinal). If FALSE, all observed endogenous variables are considered to be numeric (again, unless they are declared as ordered in the data.frame.)

sampling.weights

A variable name in the data frame containing sampling weight information. Currently only available for non-clustered data. Depending on the sampling. weights. normalization option, these weights may be rescaled (or not) so that their sum equals the number of observations (total or per group). Currently only available if estimator is ML in combination with robust standard errors and a robust test statistic. By default, the estimator will be "MLR".

sample.cov Numeric matrix. A sample variance-covariance matrix. The rownames and/or colnames must contain the observed variable names. For a multiple group analysis, a list with a variance-covariance matrix for each group.

sample.mean A sample mean vector. For a multiple group analysis, a list with a mean vector for each group.

sample.th Vector of sample-based thresholds. For a multiple group analysis, a list with a vector of thresholds for each group.

sample.nobs Number of observations if the full data frame is missing and only sample moments are given. For a multiple group analysis, a list or a vector with the number of observations for each group.

group Character. A variable name in the data frame defining the groups in a multiple group analysis.

cluster Character. A (single) variable name in the data frame defining the clusters in a two-level dataset. 
constraints Additional (in)equality constraints not yet included in the model syntax. See model. syntax for more information.

WLS.V A user provided weight matrix to be used by estimator "WLS"; if the estimator is "DWLS", only the diagonal of this matrix will be used. For a multiple group analysis, a list with a weight matrix for each group. The elements of the weight matrix should be in the following order (if all data is continuous): first the means (if a meanstructure is involved), then the lower triangular elements of the covariance matrix including the diagonal, ordered column by column. In the categorical case: first the thresholds (including the means for continuous variables), then the slopes (if any), the variances of continuous variables (if any), and finally the lower triangular elements of the correlation/covariance matrix excluding the diagonal, ordered column by column.

NACOV A user provided matrix containing the elements of ( $\mathrm{N}$ times) the asymptotic variance-covariance matrix of the sample statistics. For a multiple group analysis, a list with an asymptotic variance-covariance matrix for each group. See the WLS.V argument for information about the order of the elements.

ov.order Character. If "model" (the default), the order of the observed variable names (as reflected for example in the output of lavNames ()$)$ is determined by the model syntax. If "data", the order is determined by the data (either the full data.frame or the sample (co)variance matrix). If the WLS.V and/or NACOV matrices are provided, this argument is currently set to "data".

Many more additional options can be defined, using 'name = value'. See lav0ptions for a complete list.

\section{Details}

The cfa function is a wrapper for the more general lavaan function, using the following default arguments: int.ov.free $=$ TRUE, int.lv.free $=$ FALSE, auto.fix.first $=$ TRUE (unless std. $1 \mathrm{v}$ $=$ TRUE), auto.fix. single $=$ TRUE, auto.var $=$ TRUE, auto. cov. $1 v \cdot x=T R U E$, auto. efa $=$ TRUE, auto. th $=$ TRUE, auto. delta $=$ TRUE, and auto. cov $. y=$ TRUE.

\section{Value}

An object of class lavaan, for which several methods are available, including a summary method.

\section{References}

Yves Rosseel (2012). lavaan: An R Package for Structural Equation Modeling. Journal of Statistical Software, 48(2), 1-36. doi:10.18637/jss.v048.i02

\section{See Also}

lavaan

\section{Examples}

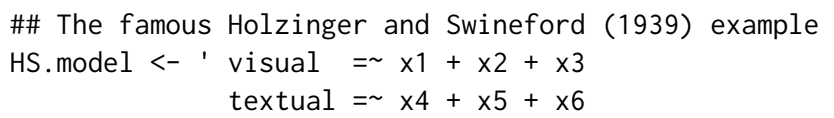




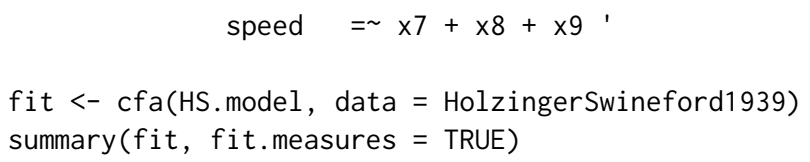

Demo.growth

Demo dataset for a illustrating a linear growth model.

\section{Description}

A toy dataset containing measures on 4 time points ( $\mathrm{t} 1, \mathrm{t} 2, \mathrm{t} 3$ and $\mathrm{t} 4)$, two predictors ( $\mathrm{x} 1$ and $\mathrm{x} 2)$ influencing the random intercept and slope, and a time-varying covariate (c1, c2, c3 and c4).

\section{Usage}

data(Demo.growth)

\section{Format}

A data frame of 400 observations of 10 variables.

t1 Measured value at time point 1

t2 Measured value at time point 2

t3 Measured value at time point 3

t4 Measured value at time point 4

$x 1$ Predictor 1 influencing intercept and slope

x2 Predictor 2 influencing intercept and slope

c1 Time-varying covariate time point 1

c2 Time-varying covariate time point 2

c3 Time-varying covariate time point 3

c4 Time-varying covariate time point 4

\section{See Also}

growth

\section{Examples}

head(Demo.growth) 


\section{Description}

A toy dataset containing measures on 6 items (y1-y6), 3 within-level covariates $(x 1-x 3)$ and 2 between-level covariates (w1-w2). The data is clustered (200 clusters of size 5, 10, 15 and 20), and the cluster variable is "cluster".

\section{Usage}

$\operatorname{data(Demo.~twolevel)~}$

\section{Format}

A data frame of 2500 observations of 12 variables. clusters.

y1 item 1

y2 item 2

y3 item 3

y4 item 4

y5 item 5

y6 item 6

$x 1$ within-level covariate 1

$x 2$ within-level covariate 2

x3 within-level covariate 3

w1 between-level covariate 1

w2 between-level covariate 2

cluster cluster variable

\section{Examples}

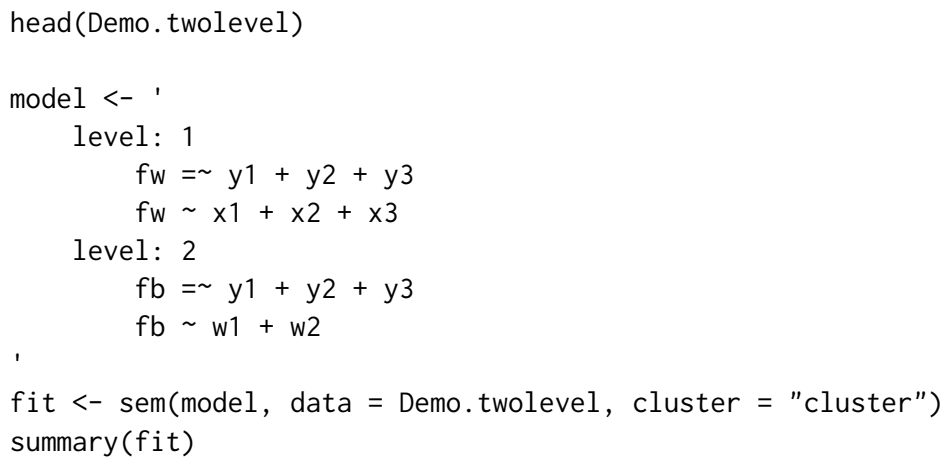


efa Exploratory Factor Analysis

\section{Description}

Fit one or more Exploratory Factor Analysis (EFA) model(s).

Usage

efa $($ data $=$ NULL, nfactors $=1 \mathrm{~L}$, sample. $\operatorname{cov}=$ NULL, sample. nobs $=$ NULL, rotation = "geomin", rotation.args $=$ list () , ov. names $=$ names $($ data $)$, bounds = "pos.var", $\ldots$, output = "efa")

\section{Arguments}

data

nfactors

sample.cov

sample.nobs

rotation

rotation.args

ov.names
A data frame containing the observed variables we need for the EFA. If only a subset of the observed variables is needed, use the ov. names argument.

Integer or Integer vector. The desired number of factors to extract. Can be a single number, or a vector of numbers (e.g., nfactors $=1: 4$.), For each different number, a model is fitted.

Numeric matrix. A sample variance-covariance matrix. The rownames and/or colnames must contain the observed variable names. Unlike sem and CFA, the matrix may be a correlation matrix.

Number of observations if the full data frame is missing and only the sample variance-covariance matrix is given.

Character. The rotation method to be used. Possible options are varimax, quartimax, orthomax, oblimin, quartimin, geomin, promax, entropy, mccammon, infomax, tandem1, tandem2, oblimax, bentler, simplimax, target, pst (=partially specified target), cf, crawford-ferguson, cf-quartimax, cf-varimax, cf-equamax, cf-parsimax, cf-facparsim, biquartimin, bigeomin. The latter two are for bifactor rotation only. The rotation algorithms (except promax) are similar to those from the GPArotation package, but have been reimplemented for better control. The promax method is taken from the stats package.

List. Options related to the rotation algorithm. The default options (and their alternatives) are or thogonal = FALSE, row. weights = "default" (or "kai ser", "cureton.mulaik" or "none"), std.ov = TRUE, algorithm = "gpa" (or "pairwise"), rstarts $=30$, gpa . tol $=1 \mathrm{e}-05$, tol $=1 \mathrm{e}-08$, max. iter $=10000 \mathrm{~L}$, warn $=$ FALSE, verbose $=$ FALSE, reflect $=$ TRUE, order $.1 \mathrm{v}$. by $=$ "index" (or "sumof squares" or "none"). Other options are specific for a particular rotation criterion: geomin. epsilon $=0.001$, or thomax .gamma $=1$, promax . kappa $=4$, cf. gamma $=0$, and oblimin. gamma $=0$.

Character vector. The variables names that are needed for the EFA. Should be a subset of the variables names in the data.frame. By default, all the variables in the data are used. 
bounds

Per default, bounds = "pos. var" forces all variances of both observed and latent variables to be strictly nonnegative. See the entry in lavoptions for more options.

$\cdots$

Aditional options to be passed to lavaan, using 'name = value'. See lavOptions for a complete list.

output

Character. If "efa" (the default), the output mimics the typical output of an EFA. If "lavaan", a lavaan object returned. The latter is only possible if nfactors contains a single (integer) number.

\section{Details}

The efa function is essentially a wrapper around the lavaan function. It generates the model syntax (for a given number of factors) and then calls lavaan() treating the factors as a single block that should be rotated. The function only supports a single group. Categorical data is handled as usual by first computing an appropriate (e.g., tetrachoric or polychoric) correlation matrix, which is then used as input for the EFA. There is also (limited) support for twolevel data. The same number of factors is then extracted at the within and the between level. The promax rotation method (taken from the stats package) is only provided for convenience. Because promax is a two-step algorithm (first varimax, then oblique rotation to get simple structure), it does not use the gpa or pairwise rotation algorithms, and as a result, no standard errors are provided.

\section{Value}

If output = "lavaan", an object of class lavaan. If output = "efa", a list of class efaList for which a print(), summary() and fitMeasures() method are available. Because we added the (standardized) loadings as an extra element, the loadings function (which is not a generic function) from the stats package will also work on efaList objects.

\section{See Also}

summary. efaList for a summary method if the output is of class efaList.

\section{Examples}

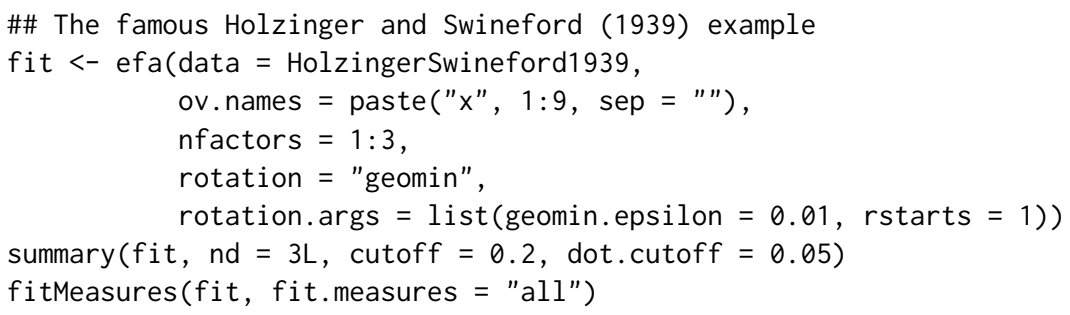




\section{Description}

A function for extracting the empirical estimating functions of a fitted lavaan model. This is the derivative of the objective function with respect to the parameter vector, evaluated at the observed (case-wise) data. In other words, this function returns the case-wise scores, evaluated at the fitted model parameters.

\section{Usage}

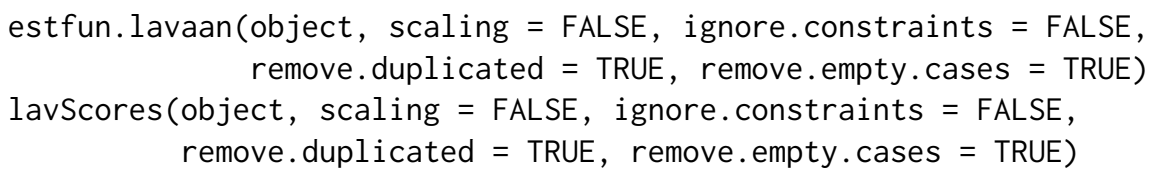

\section{Arguments}

object

An object of class lavaan.

scaling If TRUE, the scores are scaled to reflect the specific objective function used by lavaan. If FALSE (the default), the objective function is the loglikelihood function assuming multivariate normality.

ignore.constraints

Logical. If TRUE, the scores do not reflect the (equality or inequality) constraints. If FALSE, the scores are computed by taking the unconstrained scores, and adding the term $t(R)$ lambda, where lambda are the (case-wise) Lagrange Multipliers, and R is the Jacobian of the constraint function. Only in the latter case will the sum of the columns be (almost) equal to zero.

remove.duplicated

If TRUE, and all the equality constraints have a simple form (eg. $a==b$ ), the unconstrained scores are post-multiplied with a transformation matrix in order to remove the duplicated parameters.

remove.empty.cases

If TRUE, empty cases with only missing values will be removed from the output.

\section{Value}

A $\mathrm{n} x \mathrm{k}$ matrix corresponding to $\mathrm{n}$ observations and $\mathrm{k}$ parameters.

\section{Author(s)}

Ed Merkle; the remove.duplicated, ignore.constraints and remove. empty.cases arguments were added by Yves Rosseel 


\section{Description}

A dataset from the Dutch burn center (http://www.adbc.nl). The data were used to examine psychosocial functioning in patients with facial burn wounds. Psychosocial functioning was measured by Anxiety and depression symptoms (HADS), and self-esteem (Rosenberg's self-esteem scale).

\section{Usage}

data(FacialBurns)

\section{Format}

A data frame of 77 observations of 6 variables.

Selfesteem Rosenberg's self-esteem scale

HADS Anxiety and depression scale

Age Age measured in years, control variable

TBSA Total Burned Surface Area

RUM Rumination, control variable

Sex Gender, grouping variable

\section{Examples}

head(FacialBurns)

\section{Description}

This function computes a variety of fit measures to assess the global fit of a latent variable model.

\section{Usage}

$$
\begin{aligned}
& \text { fitMeasures(object, fit.measures = "all", baseline.model = NULL, } \\
& \text { fm.args = list (standard.test = "default", } \\
& \text { scaled.test } \quad=\text { "default", } \\
& \text { rmsea.ci.level }=0.90 \text {, } \\
& \text { rmsea.close.ho }=0.05 \text {, } \\
& \text { rmsea. notclose. } \mathrm{h} 0=0.08 \text {, } \\
& \text { cat. check.pd = TRUE), }
\end{aligned}
$$




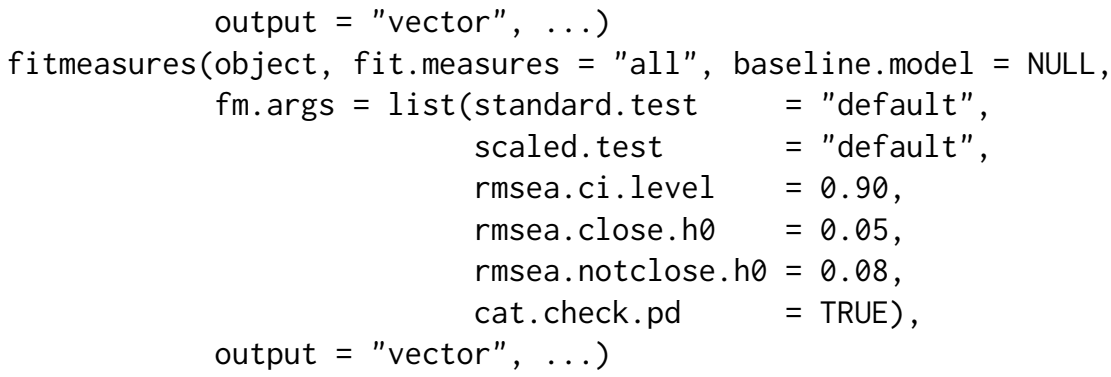

\section{Arguments}

object

fit.measures

An object of class lavaan.

If "all", all fit measures available will be returned. If only a single or a few fit measures are specified by name, only those are computed and returned.

baseline.model If not NULL, an object of class lavaan, representing a user-specified baseline model. If a baseline model is provided, all fit indices relying on a baseline model (eg. CFI or TLI) will use the test statistics from this user-specified baseline model, instead of the default baseline model.

fm.args

output

$\cdots$ determines the main test statistic (chi-square value) that will be used to compute all the fit measures that depend on this test statistic. Usually this is "standard". The scaled.test element determines which scaling method is to be used for the scaled fit measures (in case multiple scaling methods were requested). The rmsea.ci.level element determines the level of the confidence interval for the rmsea value. The rmsea. close. h0 element is the rmsea value that is used under the null hypothesis that rmsea $<=$ rmsea. close.h0. The rmsea.notclose.h0 element is the rmsea value that is used under the null hypothesis that rsmsea $>=$ rmsea. notclose.h0. The cat. check.pd element is only used when data is categorical. If TRUE, robust values for RMSEA and CFI are only computed if the input correlation matrix is positive-definite (for all groups).

Character. If "vector" (the default), display the output as a named (lavaanformatted) vector. If "matrix", display the output as a 1-column matrix. If "text", display the output using subsections and verbose descriptions. The latter is used in the summary output, and does not print the chi-square test by default. In addition, fit. measures should contain the main ingredient (for example "rmsea") if related fit measures are requested (for example "rmsea. ci. lower"). Otherwise, nothing will be printed in that section. See the examples how to add the chi-square test in the text output.

Further arguments passed to or from other methods. Not currently used for lavaan objects.
List. Additional options for certain fit measures. The standard.test element

\section{Value}

A named numeric vector of fit measures. 


\section{Examples}

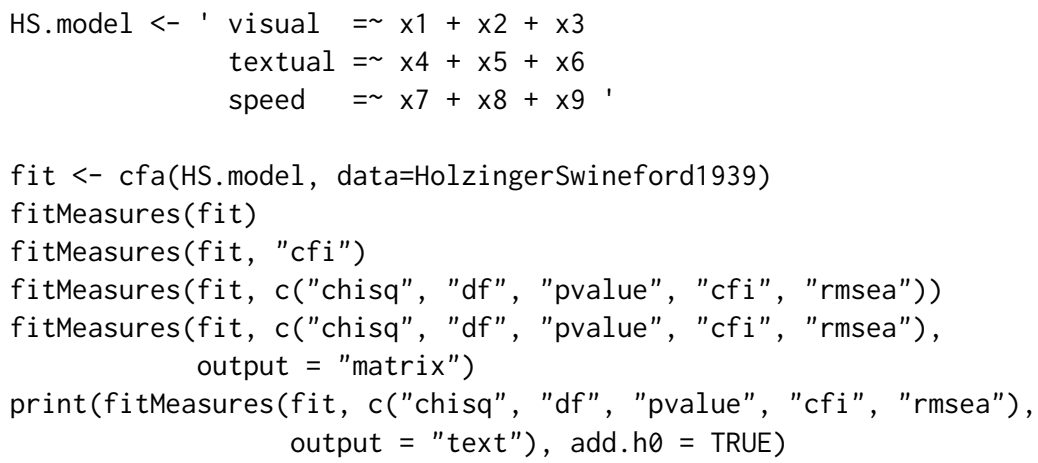

getCov Utility Functions For Covariance Matrices

\section{Description}

Convenience functions to deal with covariance and correlation matrices.

\section{Usage}

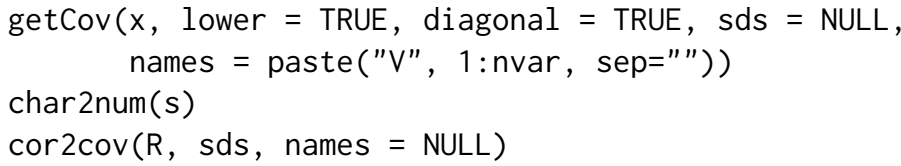

\section{Arguments}

The elements of the covariance matrix. Either inside a character string or as a numeric vector. In the former case, the function char2num is used to convert the numbers (inside the character string) to numeric values.

Logical. If TRUE, the numeric values in $x$ are the lower-triangular elements of the (symmetric) covariance matrix only. If FALSE, $x$ contains the upper triangular elements only. Note we always assumed the elements are provided row-wise!

Logical. If TRUE, the numeric values in $x$ include the diagonal elements. If FALSE, a unit diagonal is assumed.

A numeric vector containing the standard deviations to be used to scale the elements in $\mathrm{x}$ or the correlation matrix $\mathrm{R}$ into a covariance matrix.

The variable names of the observed variables.

Character string containing numeric values; comma's and semi-colons are ignored.

A correlation matrix, to be scaled into a covariance matrix. 


\section{Details}

The getCov function is typically used to input the lower (or upper) triangular elements of a (symmetric) covariance matrix. In many examples found in handbooks, only those elements are shown. However, lavaan needs a full matrix to proceed.

The cor $2 \operatorname{cov}$ function is the inverse of the cov2cor function, and scales a correlation matrix into a covariance matrix given the standard deviations of the variables. Optionally, variable names can be given.

\section{Examples}

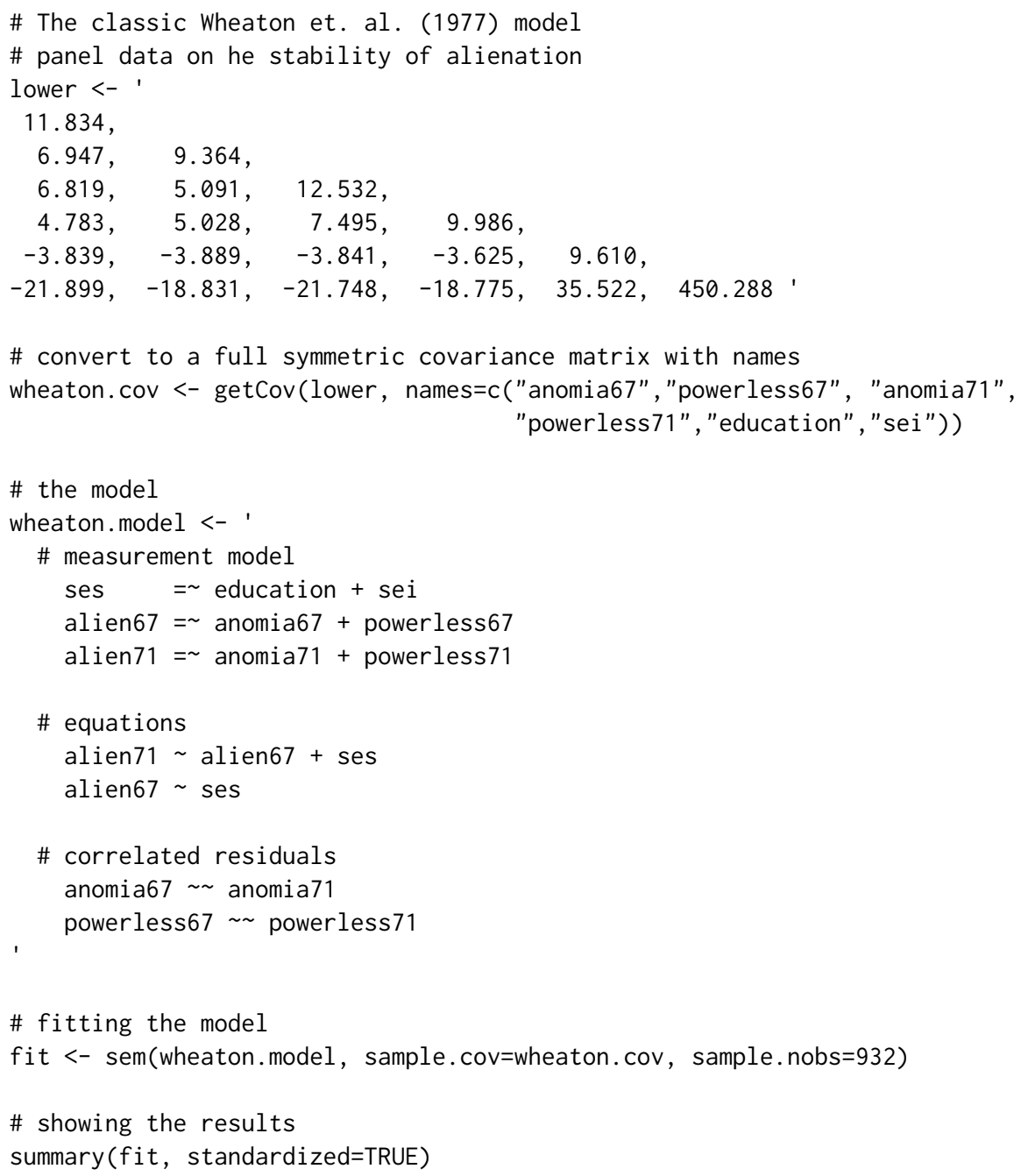




\section{Description}

Fit a Growth Curve model. Only useful if all the latent variables in the model are growth factors. For more complex models, it may be better to use the lavaan function.

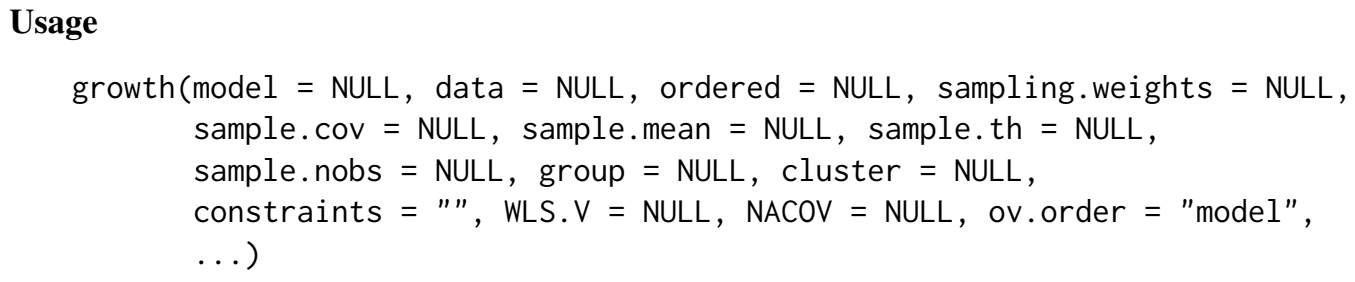

\section{Arguments}

model

data

ordered sampling. weights

A variable name in the data frame containing sampling weight information. Currently only available for non-clustered data. Depending on the sampling. weights.normalization option, these weights may be rescaled (or not) so that their sum equals the number of observations (total or per group). Currently only available if estimator is ML in combination with robust standard errors and a robust test statistic. By default, the estimator will be "MLR".

sample.cov

sample.mean

sample.th

sample.nobs

group

A description of the user-specified model. Typically, the model is described using the lavaan model syntax. See model syntax for more information. Alternatively, a parameter table (eg. the output of the lavaanify() function) is also accepted.

An optional data frame containing the observed variables used in the model. If some variables are declared as ordered factors, lavaan will treat them as ordinal variables.

Character vector. Only used if the data is in a data.frame. Treat these variables as ordered (ordinal) variables, if they are endogenous in the model. Importantly, all other variables will be treated as numeric (unless they are declared as ordered in the data.frame.) Since 0.6-4, ordered can also be logical. If TRUE, all observed endogenous variables are treated as ordered (ordinal). If FALSE, all observed endogenous variables are considered to be numeric (again, unless they are declared as ordered in the data.frame.)

Numeric matrix. A sample variance-covariance matrix. The rownames and/or colnames must contain the observed variable names. For a multiple group analysis, a list with a variance-covariance matrix for each group.

A sample mean vector. For a multiple group analysis, a list with a mean vector for each group.

Vector of sample-based thresholds. For a multiple group analysis, a list with a vector of thresholds for each group.

Number of observations if the full data frame is missing and only sample moments are given. For a multiple group analysis, a list or a vector with the number of observations for each group.

Character. A variable name in the data frame defining the groups in a multiple group analysis. 
cluster Character. A (single) variable name in the data frame defining the clusters in a two-level dataset.

constraints Additional (in)equality constraints not yet included in the model syntax. See model. syntax for more information.

WLS.V A user provided weight matrix to be used by estimator "WLS"; if the estimator is "DWLS", only the diagonal of this matrix will be used. For a multiple group analysis, a list with a weight matrix for each group. The elements of the weight matrix should be in the following order (if all data is continuous): first the means (if a meanstructure is involved), then the lower triangular elements of the covariance matrix including the diagonal, ordered column by column. In the categorical case: first the thresholds (including the means for continuous variables), then the slopes (if any), the variances of continuous variables (if any), and finally the lower triangular elements of the correlation/covariance matrix excluding the diagonal, ordered column by column.

NACOV A user provided matrix containing the elements of ( $\mathrm{N}$ times) the asymptotic variance-covariance matrix of the sample statistics. For a multiple group analysis, a list with an asymptotic variance-covariance matrix for each group. See the WLS. V argument for information about the order of the elements.

ov.order Character. If "model" (the default), the order of the observed variable names (as reflected for example in the output of lavNames ()$)$ is determined by the model syntax. If "data", the order is determined by the data (either the full data.frame or the sample (co)variance matrix). If the WLS.V and/or NACOV matrices are provided, this argument is currently set to "data".

Many more additional options can be defined, using 'name = value'. See lav0ptions for a complete list.

\section{Details}

The growth function is a wrapper for the more general lavaan function, using the following default arguments: meanstructure $=T R U E$, int.ov.free $=$ FALSE, int.lv.free $=$ TRUE, auto.fix.first $=$ TRUE (unless std.lv= TRUE), auto.fix.single = TRUE, auto.var $=$ TRUE, auto.cov.lv.x $=$ TRUE, auto. efa = TRUE, auto. th = TRUE, auto. delta $=$ TRUE, and auto. cov. $y=$ TRUE.

\section{Value}

An object of class lavaan, for which several methods are available, including a summary method.

\section{References}

Yves Rosseel (2012). lavaan: An R Package for Structural Equation Modeling. Journal of Statistical Software, 48(2), 1-36. doi:10.18637/jss.v048.i02

\section{See Also}

lavaan 


\title{
Examples
}

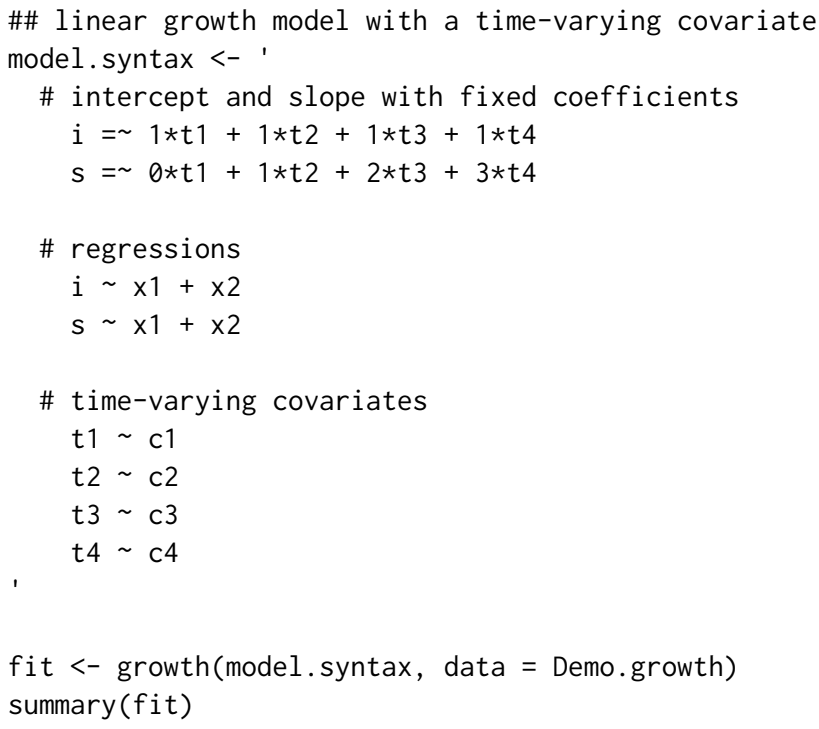

HolzingerSwineford1939

\author{
Holzinger and Swineford Dataset (9 Variables)
}

\section{Description}

The classic Holzinger and Swineford (1939) dataset consists of mental ability test scores of seventhand eighth-grade children from two different schools (Pasteur and Grant-White). In the original dataset (available in the MBESS package), there are scores for 26 tests. However, a smaller subset with 9 variables is more widely used in the literature (for example in Joreskog's 1969 paper, which also uses the 145 subjects from the Grant-White school only).

\section{Usage}

data(HolzingerSwineford1939)

\section{Format}

A data frame with 301 observations of 15 variables.

id Identifier

sex Gender

ageyr Age, year part

agemo Age, month part

school School (Pasteur or Grant-White)

grade Grade 
$\mathrm{x} 1$ Visual perception

$\times 2$ Cubes

$x 3$ Lozenges

$\times 4$ Paragraph comprehension

$x 5$ Sentence completion

$x 6$ Word meaning

$x 7$ Speeded addition

$x 8$ Speeded counting of dots

$\times 9$ Speeded discrimination straight and curved capitals

\section{Source}

This dataset was originally retrieved from http://web. missouri.edu/ kolenikovs/stata/hs-cfa.dta (link no longer active) and converted to an $\mathrm{R}$ dataset.

\section{References}

Holzinger, K., and Swineford, F. (1939). A study in factor analysis: The stability of a bifactor solution. Supplementary Educational Monograph, no. 48. Chicago: University of Chicago Press.

Joreskog, K. G. (1969). A general approach to confirmatory maximum likelihood factor analysis. Psychometrika, 34, 183-202.

\section{See Also}

cfa

\section{Examples}

head(HolzingerSwineford1939)

InformativeTesting

Testing order/inequality Constrained Hypotheses in SEM

\section{Description}

Testing order/inequality constrained Hypotheses in SEM

\section{Usage}

InformativeTesting (model $=$ NULL, data, constraints $=$ NULL,

$\mathrm{R}=1000 \mathrm{~L}$, type = "bollen.stine",

return.LRT = TRUE,

double. bootstrap = "standard",

double. bootstrap. $\mathrm{R}=249 \mathrm{~L}$,

double. bootstrap.alpha $=0.05$,

parallel = c("no", "multicore", "snow"),

ncpus $=1 \mathrm{~L}, \mathrm{cl}=\mathrm{NULL}$, verbose $=$ FALSE,$\ldots$ ) 


\section{Arguments}

\begin{tabular}{|c|c|}
\hline model & Model syntax specifying the model. See model. syntax for more information. \\
\hline data & The data frame containing the observed variables being used to fit the model. \\
\hline constraints & The imposed inequality constraints on the model. \\
\hline $\mathrm{R}$ & Integer; number of bootstrap draws. The default value is set to 1000 . \\
\hline type & $\begin{array}{l}\text { If "parametric", the parametric bootstrap is used. If "bollen. stine", the } \\
\text { semi-nonparametric Bollen-Stine bootstrap is used. The default is set to "bollen. stine". }\end{array}$ \\
\hline return.LRT & Logical; if TRUE, the function returns bootstrapped LRT-values. \\
\hline \multicolumn{2}{|c|}{ double. bootstrap } \\
\hline & $\begin{array}{l}\text { If "standard" (default) the genuine double bootstrap is used to compute an } \\
\text { additional set of plug-in p-values for each bootstrap sample. If "no", no double } \\
\text { bootstrap is used. If "FDB", the fast double bootstrap is used to compute second } \\
\text { level LRT-values for each bootstrap sample. Note that the "FDB" is experimental } \\
\text { and should not be used by inexperienced users. }\end{array}$ \\
\hline \multicolumn{2}{|c|}{ double.bootstrap.R } \\
\hline & Integer; number of double bootstrap draws. The default value is set to 249 . \\
\hline \multicolumn{2}{|c|}{ double.bootstrap.alpha } \\
\hline & $\begin{array}{l}\text { The significance level to compute the adjusted alpha based on the plugin p- } \\
\text { values. Only used if double. bootstrap = "standard". The default value is } \\
\text { set to } 0.05 \text {. }\end{array}$ \\
\hline parallel & $\begin{array}{l}\text { The type of parallel operation to be used (if any). If missing, the default is set } \\
\text { "no". }\end{array}$ \\
\hline ncpus & $\begin{array}{l}\text { Integer: number of processes to be used in parallel operation: typically one } \\
\text { would chose this to the number of available CPUs. }\end{array}$ \\
\hline cl & $\begin{array}{l}\text { An optional parallel or snow cluster for use if parallel = "snow". If not sup- } \\
\text { plied, a cluster on the local machine is created for the duration of the InformativeTesting } \\
\text { call. }\end{array}$ \\
\hline verbose & Logical; if TRUE, information is shown at each bootstrap draw. \\
\hline & $\begin{array}{l}\text { Other named arguments from the lavaan package which are passed to the func- } \\
\text { tion. For example "group" in a multiple group model. }\end{array}$ \\
\hline
\end{tabular}

\section{Details}

The following hypothesis tests are available:

- Type A: Test H0: all restriktions with equalities ("=") active against HA: at least one inequality restriktion (">") strictly true.

- Type B: Test H0: all restriktions with inequalities (">") (including some equalities ("=")) active against HA: at least one restriktion false (some equality restriktions may be maintained).

\section{Value}

An object of class InformativeTesting for which a print and a plot method is available. 


\section{$\operatorname{Author}(\mathbf{s})$}

Leonard Vanbrabant <lgf. vanbrabant@gmail. com>

\section{References}

Van de Schoot, R., Hoijtink, H., \& Dekovic, M. (2010). Testing inequality constrained hypotheses in SEM models. Structural Equation Modeling, 17, 443-463.

Van de Schoot, R., Strohmeier, D. (2011). Testing informative hypotheses in SEM increases power: An illustration contrasting classical. International Journal of Behavioral Development, 35, 180190.

Silvapulle, M.J. and Sen, P.K. (2005). Constrained Statistical Inference. Wiley, New York.

\section{Examples}

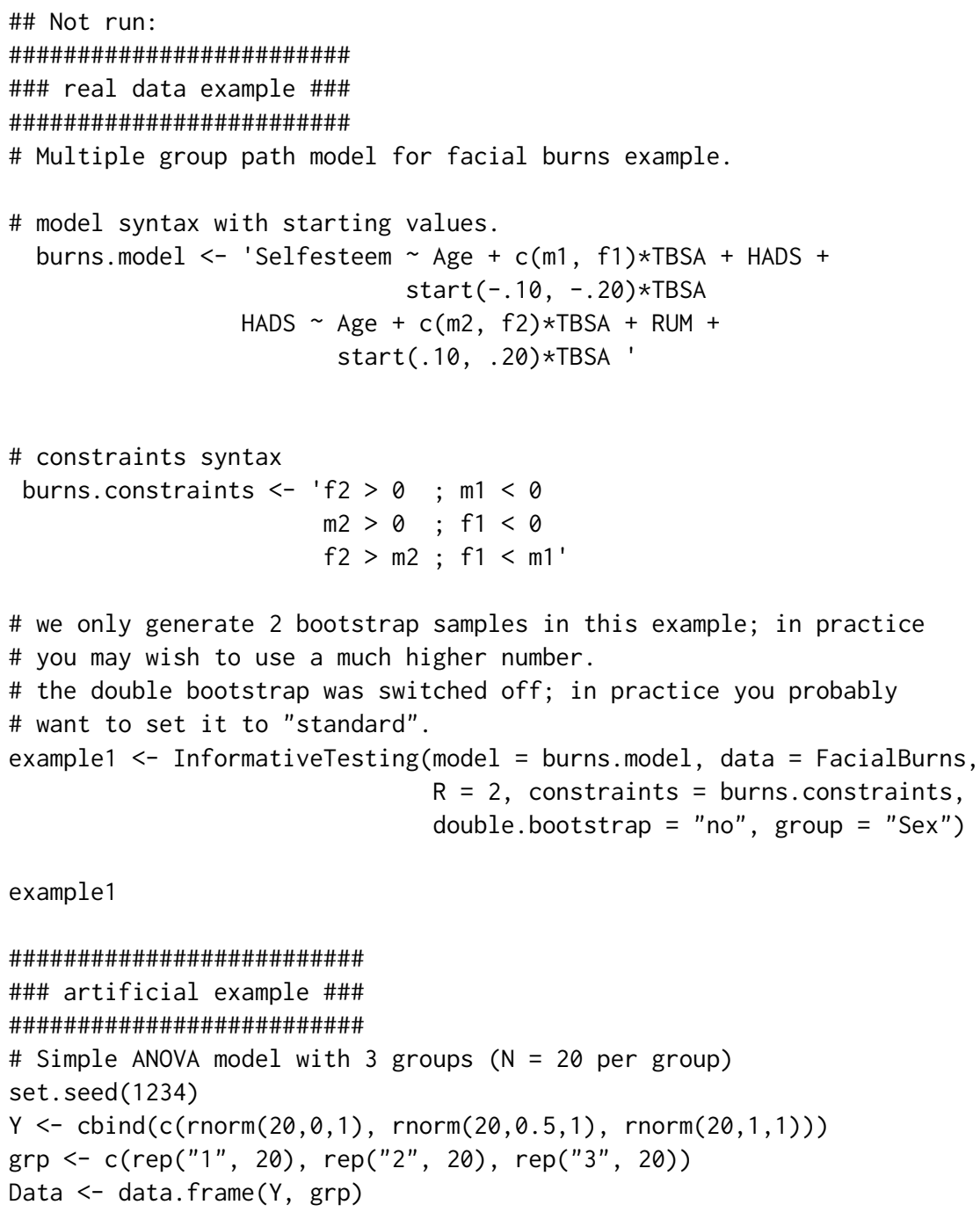




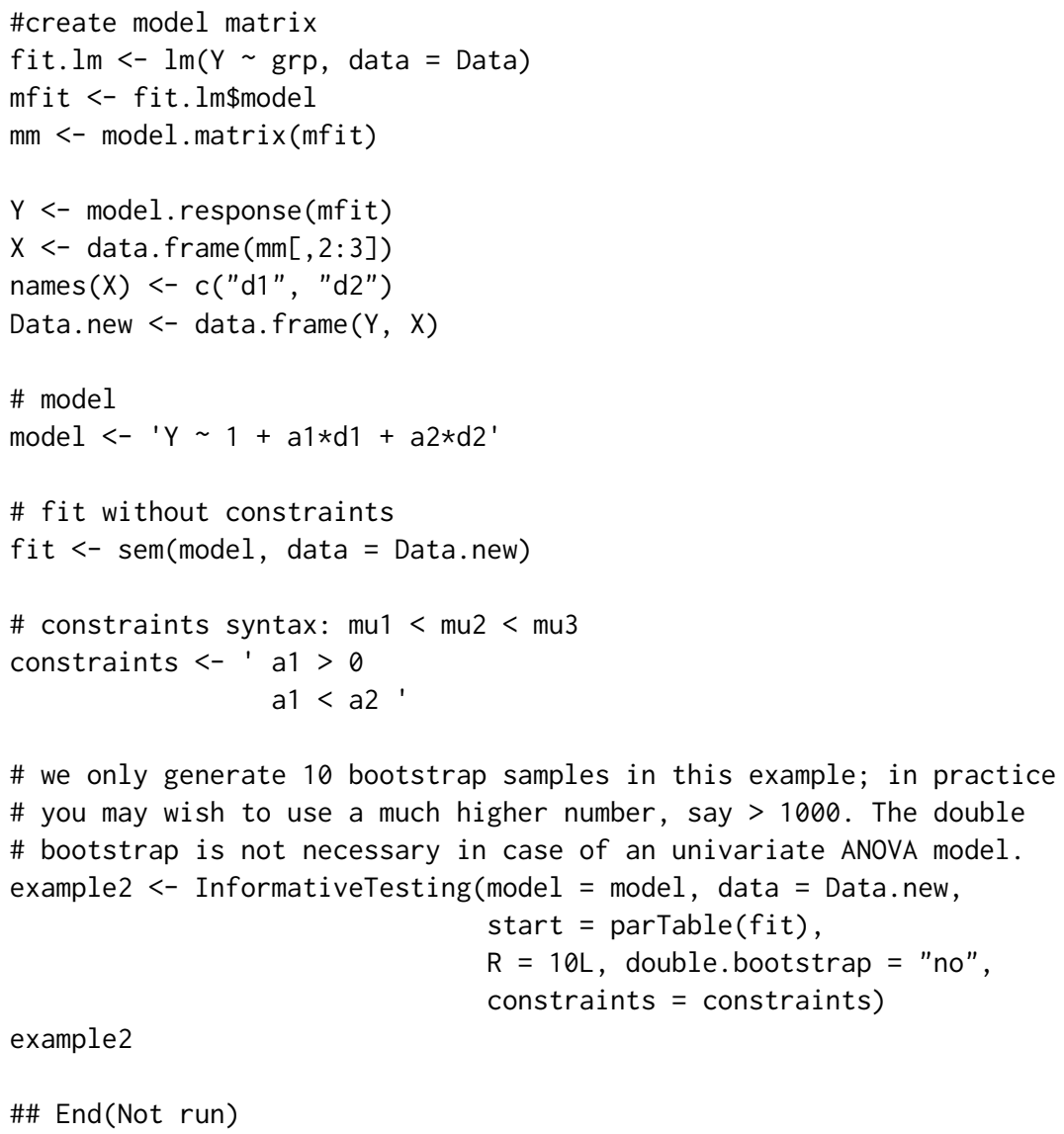

InformativeTesting methods

Methods for output InformativeTesting()

\section{Description}

The print function shows the results of hypothesis tests Type A and Type B. The plot function plots the distributions of bootstrapped LRT values and plug-in p-values.

\section{Usage}

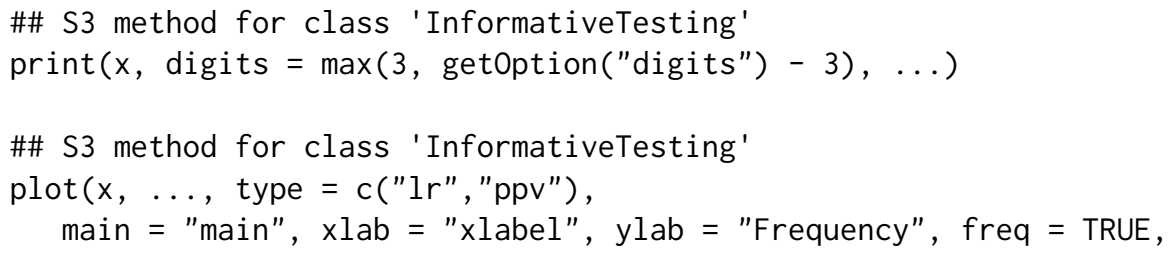


breaks $=15$, cex.main $=1$, cex.lab $=1$, cex. axis $=1$, col = "grey", border = par ("fg"), vline = TRUE, vline.col =c("red", "blue"), lty $=c(1,2)$, lwd $=1$, legend $=$ TRUE, bty $=$ "o", cex.legend $=1$, loc.legend = "topright")

\section{Arguments}

$\mathrm{X}$

digits

..

type

main

xlab

ylab

freq

breaks

cex.main

cex. 1 ab

cex.axis

col

border

vline

vline.col

1ty

Iwd

legend

bty object of class "InformativeTesting".

the number of significant digits to use when printing.

Currently not used.

If " $r$ ", a distribution of the first-level bootstrapped LR values is plotted. If "ppv" a distribution of the bootstrapped plug-in p-values is plotted.

The main title(s) for the plot(s).

A label for the $\mathrm{x}$ axis, default depends on input type.

A label for the $y$ axis.

Logical; if TRUE, the histogram graphic is a representation of frequencies, the counts component of the result; if FALSE, probability densities, component density, are plotted (so that the histogram has a total area of one). The default is set to TRUE.

see hist

The magnification to be used for main titles relative to the current setting of cex.

The magnification to be used for $\mathrm{x}$ and $\mathrm{y}$ labels relative to the current setting of cex.

The magnification to be used for axis annotation relative to the current setting of cex.

A colour to be used to fill the bars. The default of NULL yields unfilled bars.

Color for rectangle border(s). The default means par("fg").

Logical; if TRUE a vertical line is drawn at the observed LRT value. If double . bootstrap

$=$ "FDB" a vertical line is drawn at the 1-p* quantile of the second-level LRT values, where $\mathrm{p}^{*}$ is the first-level bootstrapped p-value

Color(s) for the vline.LRT.

The line type. Line types can either be specified as an integer $(0=$ blank, $1=$ solid (default), 2=dashed, 3=dotted, 4=dotdash, 5=longdash, $6=$ twodash) or as one of the character strings "blank", "solid", "dashed", "dotted", "dotdash", "longdash", or "twodash", where "blank" uses 'invisible lines' (i.e., does not draw them).

The line width, a positive number, defaulting to 1 .

Logical; if TRUE a legend is added to the plot.

A character string which determined the type of box which is drawn about plots. If bty is one of "o" (the default), "l", "7", "c", "u", or "]" the resulting box resembles the corresponding upper case letter. A value of " $n$ " suppresses the box. 
cex. legend

A numerical value giving the amount by which the legend text and symbols should be magnified relative to the default. This starts as 1 when a device is opened, and is reset when the layout is changed.

loc.legend The location of the legend, specified by a single keyword from the list "bottomright", "bottom", "bottomleft", "left", "topleft", "top", "topright", "right" and "center".

\section{Author(s)}

Leonard Vanbrabant <lgf.vanbrabant@gmail.com>

\section{Examples}

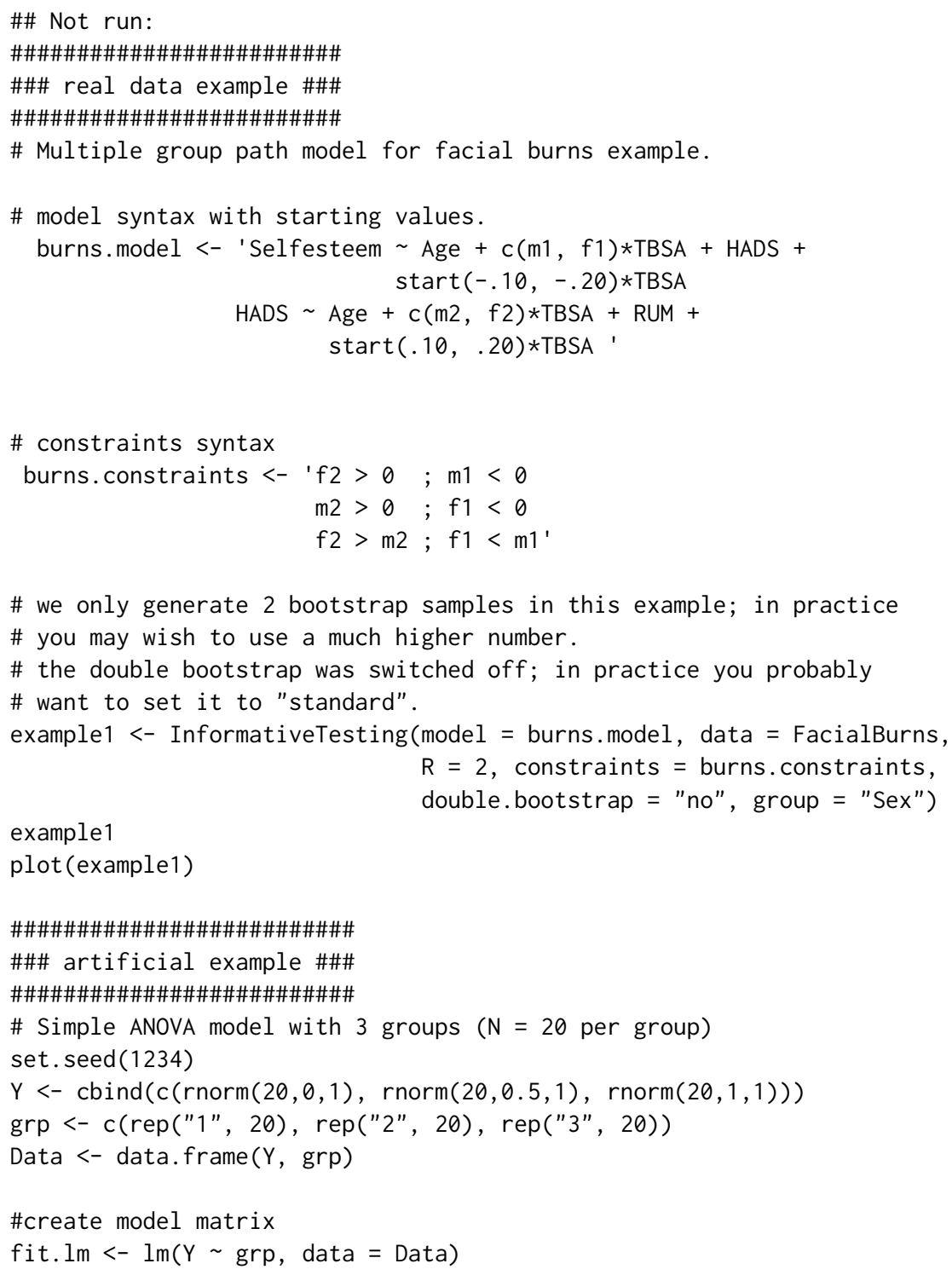




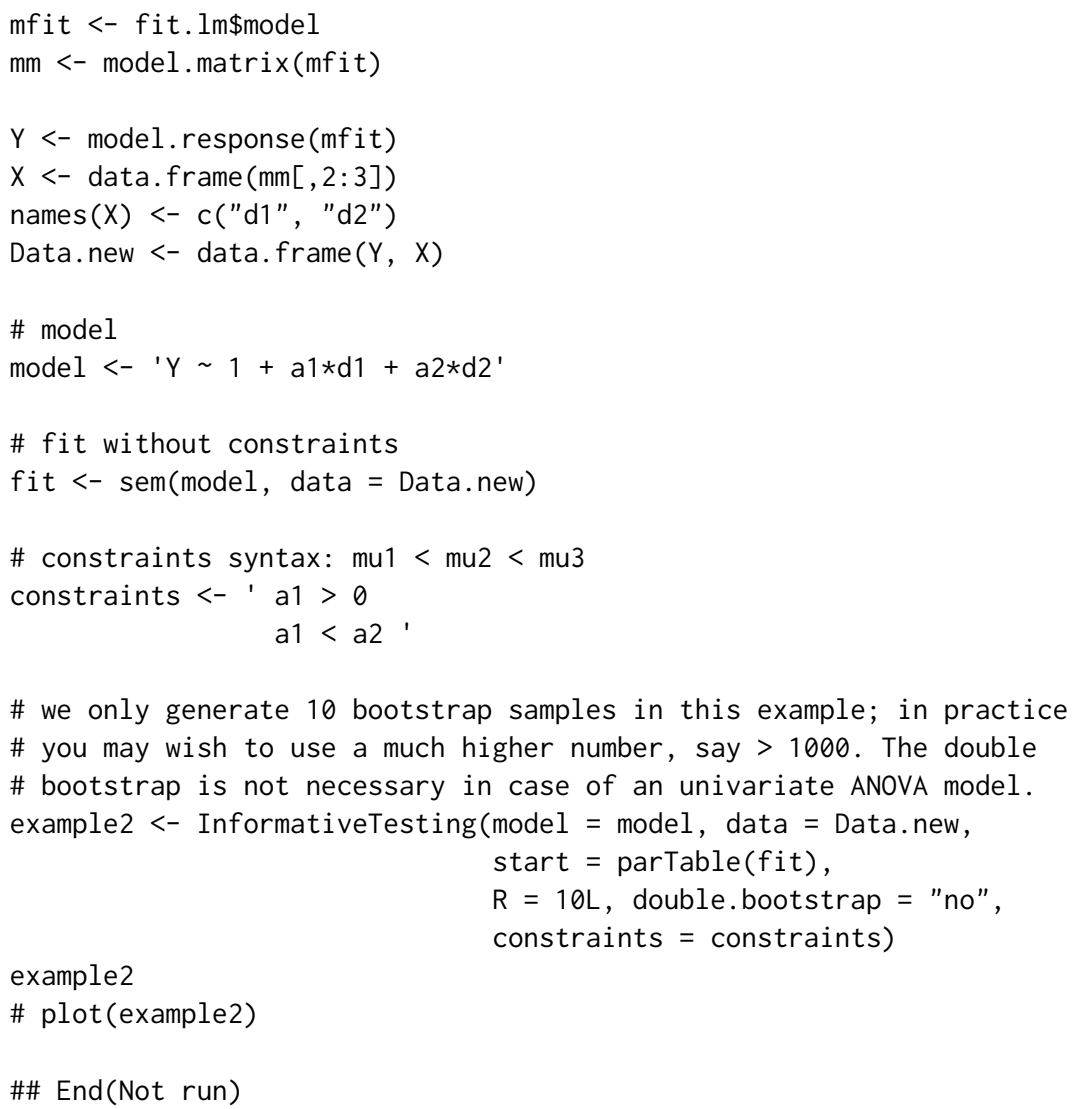

\section{Description}

The lavaan model syntax describes a latent variable model. Often, the user wants to see the covariance matrix generated by their model for diagnostic purposes. However, their data may have far more columns of information than what is contained in their model.

\section{Usage}

inspectSampleCov(model, data, ...)

\section{Arguments}

model The model that will be fit by lavaan.

data The data frame being used to fit the model.

... Other arguments to sem for how to deal with multiple groups, missing values, etc. 


\section{Details}

One must supply both a model, coded with proper model. syntax and a data frame from which a covariance matrix will be calculated. This function essentially calls sem, but doesn't fit the model, then uses lavInspect to get the sample covariance matrix and meanstructure.

See also

sem, lavInspect

Author(s)

Jarrett Byrnes

lavaan

Fit a Latent Variable Model

\section{Description}

Fit a latent variable model.

\section{Usage}

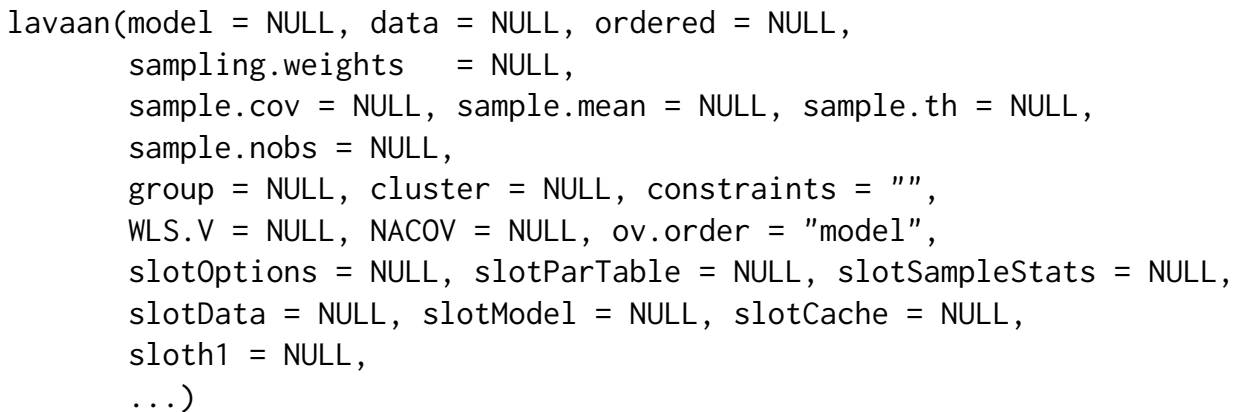

\section{Arguments}

model

data

ordered
A description of the user-specified model. Typically, the model is described using the lavaan model syntax. See model syntax for more information. Alternatively, a parameter table (eg. the output of the lavaanify() function) is also accepted.

An optional data frame containing the observed variables used in the model. If some variables are declared as ordered factors, lavaan will treat them as ordinal variables.

Character vector. Only used if the data is in a data.frame. Treat these variables as ordered (ordinal) variables, if they are endogenous in the model. Importantly, all other variables will be treated as numeric (unless they are declared as ordered in the data.frame.) Since 0.6-4, ordered can also be logical. If TRUE, all observed endogenous variables are treated as ordered (ordinal). If FALSE, all 
observed endogenous variables are considered to be numeric (again, unless they are declared as ordered in the data.frame.)

sampling. weights

A variable name in the data frame containing sampling weight information. Currently only available for non-clustered data. Depending on the sampling. weights. normalization option, these weights may be rescaled (or not) so that their sum equals the number of observations (total or per group). Currently only available if estimator is ML in combination with robust standard errors and a robust test statistic. By default, the estimator will be "MLR".

sample.cov Numeric matrix. A sample variance-covariance matrix. The rownames and/or colnames must contain the observed variable names. For a multiple group analysis, a list with a variance-covariance matrix for each group.

sample.mean A sample mean vector. For a multiple group analysis, a list with a mean vector for each group.

sample.th Vector of sample-based thresholds. For a multiple group analysis, a list with a vector of thresholds for each group.

sample.nobs Number of observations if the full data frame is missing and only sample moments are given. For a multiple group analysis, a list or a vector with the number of observations for each group.

group Character. A variable name in the data frame defining the groups in a multiple group analysis.

cluster Character. A (single) variable name in the data frame defining the clusters in a two-level dataset.

constraints Additional (in)equality constraints not yet included in the model syntax. See model. syntax for more information.

WLS.V A user provided weight matrix to be used by estimator "WLS"; if the estimator is "DWLS", only the diagonal of this matrix will be used. For a multiple group analysis, a list with a weight matrix for each group. The elements of the weight matrix should be in the following order (if all data is continuous): first the means (if a meanstructure is involved), then the lower triangular elements of the covariance matrix including the diagonal, ordered column by column. In the categorical case: first the thresholds (including the means for continuous variables), then the slopes (if any), the variances of continuous variables (if any), and finally the lower triangular elements of the correlation/covariance matrix excluding the diagonal, ordered column by column.

NACOV A user provided matrix containing the elements of ( $\mathrm{N}$ times) the asymptotic variance-covariance matrix of the sample statistics. For a multiple group analysis, a list with an asymptotic variance-covariance matrix for each group. See the WLS.V argument for information about the order of the elements.

ov.order Character. If "model" (the default), the order of the observed variable names (as reflected for example in the output of lavNames () ) is determined by the model syntax. If "data", the order is determined by the data (either the full data.frame or the sample (co)variance matrix). If the WLS.V and/or NACOV matrices are provided, this argument is currently set to "data".

slotoptions Options slot from a fitted lavaan object. If provided, no new Options slot will be created by this call. 
slotParTable ParTable slot from a fitted lavaan object. If provided, no new ParTable slot will be created by this call.

slotSampleStats

SampleStats slot from a fitted lavaan object. If provided, no new SampleStats slot will be created by this call.

slotData Data slot from a fitted lavaan object. If provided, no new Data slot will be created by this call.

slotModel Model slot from a fitted lavaan object. If provided, no new Model slot will be created by this call.

slotCache Cache slot from a fitted lavaan object. If provided, no new Cache slot will be created by this call.

sloth1 h1 slot from a fitted lavaan object. If provided, no new h1 slot will be created by this call.

... Many more additional options can be defined, using 'name = value'. See lav0ptions for a complete list.

\section{Value}

An object of class lavaan, for which several methods are available, including a summary method.

\section{References}

Yves Rosseel (2012). lavaan: An R Package for Structural Equation Modeling. Journal of Statistical Software, 48(2), 1-36. doi:10.18637/jss.v048.i02

\section{See Also}

cfa, sem, growth

\section{Examples}

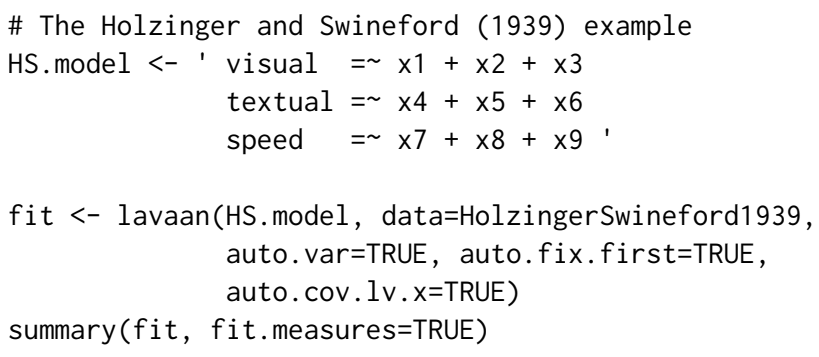




\section{Description}

The lavaan class represents a (fitted) latent variable model. It contains a description of the model as specified by the user, a summary of the data, an internal matrix representation, and if the model was fitted, the fitting results.

\section{Objects from the Class}

Objects can be created via the cfa, sem, growth or lavaan functions.

\section{Slots}

version: The lavaan package version used to create this objects

call: The function call as returned by match.call().

timing: The elapsed time (user+system) for various parts of the program as a list, including the total time.

Options: Named list of options that were provided by the user, or filled-in automatically.

ParTable: Named list describing the model parameters. Can be coerced to a data.frame. In the documentation, this is called the 'parameter table'.

pta: Named list containing parameter table attributes.

Data: Object of internal class "Data": information about the data.

SampleStats: Object of internal class "SampleStats": sample statistics

Model: Object of internal class "Model": the internal (matrix) representation of the model

Cache: List using objects that we try to compute only once, and reuse many times.

Fit: Object of internal class "Fit": the results of fitting the model. No longer used.

boot: List. Results and information about the bootstrap.

optim: List. Information about the optimization.

loglik: List. Information about the loglikelihood of the model (if maximum likelihood was used). implied: List. Model implied statistics.

vcov: List. Information about the variance matrix (vcov) of the model parameters.

test: List. Different test statistics.

h1: List. Information about the unrestricted h1 model (if available).

baseline: List. Information about a baseline model (often the independence model) (if available).

internal: List. For internal use only.

external: List. Empty slot to be used by add-on packages. 
Methods

coef signature (object = "lavaan", type = "free"): Returns the estimates of the parameters in the model as a named numeric vector. If type $=$ "free", only the free parameters are returned. If type="user", all parameters listed in the parameter table are returned, including constrained and fixed parameters.

fitted.values signature (object = "lavaan"): Returns the implied moments of the model as a list with two elements (per group): cov for the implied covariance matrix, and mean for the implied mean vector. If only the covariance matrix was analyzed, the implied mean vector will be zero.

fitted signature (object = "lavaan"): an alias for fitted.values.

residuals signature (object $=$ "lavaan", type="raw"): If type $=$ "raw", this function returns the raw (= unscaled) difference between the observed and the expected (model-implied) summary statistics. If type = "cor", or type = "cor .bollen", the observed and model implied covariance matrices are first transformed to a correlation matrix (using cov2cor () ), before the residuals are computed. If type = "cor . bentler", both the observed and model implied covariance matrices are rescaled by dividing the elements by the square roots of the corresponding variances of the observed covariance matrix. If type="normalized", the residuals are divided by the square root of the asymptotic variance of the corresponding summary statistic (the variance estimate depends on the choice for the se argument). Unfortunately, the corresponding normalized residuals are not entirely correct, and this option is only available for historical interest. If type="standardized", the residuals are divided by the square root of the asymptotic variance of these residuals. The resulting standardized residuals elements can be interpreted as z-scores. If type="standardized.mplus", the residuals are divided by the square root of the asymptotic variance of these residuals. However, a simplified formula is used (see the Mplus reference below) which often results in negative estimates for the variances, resulting in many NA values for the standardized residuals.

resid signature (object $=$ "lavaan"): an alias for residuals

vcov signature (object = "lavaan"): returns the covariance matrix of the estimated parameters.

predict signature $($ object $=$ "lavaan"): compute factor scores for all cases that are provided in the data frame. For complete data only.

anova signature (object = "lavaan"): returns model comparison statistics. This method is just a wrapper around the function lavTestLRT. If only a single argument (a fitted model) is provided, this model is compared to the unrestricted model. If two or more arguments (fitted models) are provided, the models are compared in a sequential order. Test statistics are based on the likelihood ratio test. For more details and further options, see the lavTestLRT page.

update signature (object $=$ "lavaan", model, add, . . , evaluate=TRUE): update a fitted lavaan object and evaluate it (unless evaluate=FALSE). Note that we use the environment that is stored within the lavaan object, which is not necessarily the parent frame. The add argument is analogous to the one described in the lavTestScore page, and can be used to add parameters to the specified model rather than passing an entirely new model argument.

nobs signature (object $=$ "lavaan"): returns the effective number of observations used when fitting the model. In a multiple group analysis, this is the sum of all observations per group.

logLik signature (object = "lavaan"): returns the log-likelihood of the fitted model, if maximum likelihood estimation was used. The AIC and BIC methods automatically work via $\log \operatorname{Lik}()$. 
show signature (object $=$ "lavaan"): Print a short summary of the model fit

summary signature (object = "lavaan", header = TRUE, fit. measures = FALSE, estimates

$=$ TRUE, $\mathrm{ci}=$ FALSE, $\mathrm{fmi}=$ FALSE, standardized $=$ FALSE, remove. step1 $=$ TRUE, cov. std

$=$ TRUE, $r$ square $=$ FALSE, std. nox $=$ FALSE, modindices $=$ FALSE, $c i=F A L S E, n d=3 L):$ Print

a nice summary of the model estimates. If header $=$ TRUE, the header section (including fit measures) is printed. If fit.measures = TRUE, additional fit measures are added to the header section. The related $\mathrm{fm}$. args list allows to set options related to the fit measures. See fitMeasures for more details. If estimates = TRUE, print the parameter estimates section. If $\mathrm{ci}=$ TRUE, add confidence intervals to the parameter estimates section. If $\mathrm{fmi}=\mathrm{TRUE}$, add the fmi (fraction of missing information) column, if it is available. If standardized=TRUE, the standardized solution is also printed. Note that $S E$ s and tests are still based on unstandardized estimates. Use standardizedSolution to obtain $S E$ s and test statistics for standardized estimates. If remove.step1, the parameters of the measurement part are not shown (only used when using sam().) If rsquare=TRUE, the R-Square values for the dependent variables in the model are printed. If std. nox = TRUE, the std. all column contains the the std. nox column from the parameterEstimates() output. If efa = TRUE, EFA related information is printed. The related efa.args list allows to set options related to the EFA output. See summary. efaList for more details. If modindices=TRUE, modification indices are printed for all fixed parameters. The argument nd determines the number of digits after the decimal point to be printed (currently only in the parameter estimates section.) Historically, nothing was returned, but since 0.6-12, a list is returned of class lavaan. summary for which is print function is available.

\section{References}

Yves Rosseel (2012). lavaan: An R Package for Structural Equation Modeling. Journal of Statistical Software, 48(2), 1-36. doi:10.18637/jss.v048.i02

Standardized Residuals in Mplus. Document retrieved from URL https:/www.statmodel.com/download/StandardizedResidu

\section{See Also}

cfa, sem, fitMeasures, standardizedSolution, parameterEstimates, lavInspect, modindices

\section{Examples}

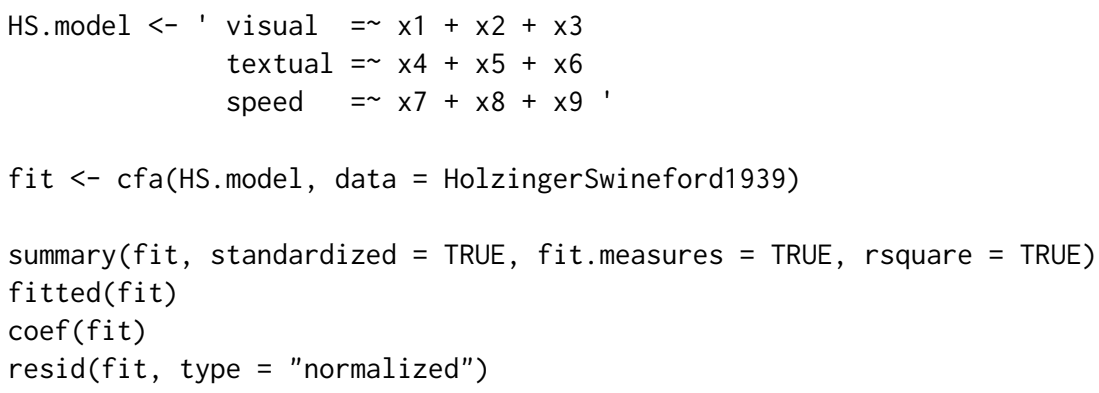




\section{Description}

Fit the same latent variable model, for a (potentially large) number of datasets.

\section{Usage}

lavaanList (model $=$ NULL, dataList $=$ NULL, dataFunction $=$ NULL, dataFunction.args $=$ list (), ndat $=$ length(dataList), $\mathrm{cmd}=$ "lavaan", $\ldots$, store.slots $=c("$ partable" $), F U N=$ NULL, show.progress $=$ FALSE, store.failed = FALSE, parallel = c("no", "multicore", "snow"), ncpus $=\max (1 \mathrm{~L}$, parallel: $: \operatorname{detectCores}()-1 \mathrm{~L}), \mathrm{cl}=\mathrm{NULL}$, iseed $=\mathrm{NULL})$

semList (model $=$ NULL, dataList $=$ NULL, dataFunction $=$ NULL, dataFunction.args $=$ list () , ndat $=$ length(dataList), ..., store.slots $=c($ "partable"), FUN = NULL, show.progress $=$ FALSE, store.failed = FALSE, parallel = c("no", "multicore", "snow"), ncpus $=\max (1 \mathrm{~L}$, parallel: $: \operatorname{detectCores}()-1 \mathrm{~L}), \mathrm{cl}=\mathrm{NULL}$, iseed $=\mathrm{NULL})$

cfaList (model $=$ NULL, dataList $=$ NULL, dataFunction $=$ NULL, dataFunction.args $=$ list () , ndat = length(dataList), $\ldots$, store.slots $=c($ "partable"), FUN $=$ NULL, show.progress $=$ FALSE, store.failed $=$ FALSE, parallel = c("no", "multicore", "snow"), ncpus $=\max (1 \mathrm{~L}$, parallel: $: \operatorname{detectCores}()-1 \mathrm{~L}), \mathrm{cl}=\mathrm{NULL}$, iseed $=$ NULL)

\section{Arguments}

model

A description of the user-specified model. Typically, the model is described using the lavaan model syntax. See model. syntax for more information. Alternatively, a parameter table (eg. the output of the lavaanify () function) is also accepted.

dataList List. Each element contains a full data frame containing the observed variables used in the model.

dataFunction Function. A function that generated a full data frame containing the observed variables used in the model. It can also be a matrix, if the columns are named.

dataFunction.args

List. Optional list of arguments that are passed to the dataFunction function.

ndat Integer. The number of datasets that should be generated using the dataFunction function.

cmd

Character. Which command is used to run the sem models. The possible choices are "sem", "cfa" or "lavaan", determining how we deal with default options.

Other named arguments for lavaan function. 
store.slots

FUN

store.failed

parallel

ncpus

cl

iseed

show.progress
Character vector. Which slots (from a lavaan object) should be stored for each dataset? The possible choices are "timing", "partable", "data", "samplestats", "vcov", "test", "optim", "h1", "loglik", or "implied". Finally, "all" selects all slots.

Function. A function which when applied to the lavaan object returns the information of interest.

Logical. If TRUE, write (to tempdir()) the dataset and (if available) the fitted object when the estimation for a particular dataset somehow failed. This will allow posthoc inspection of the problem.

The type of parallel operation to be used (if any). If missing, the default is "no".

Integer. The number of processes to be used in parallel operation: typically one would chose this to the number of available CPUs.

An optional parallel or snow cluster for use if parallel = "snow". If not supplied, a cluster on the local machine is created for the duration of the lavaanList call.

An integer to set the seed. Or NULL if no reproducible seeds are needed. To make this work, make sure the first RNGkind() element is "L 'Ecuyer-CMRG". You can check this by typing RNGkind() in the console. You can set it by typing RNGkind("L'Ecuyer-CMRG"), before the lavaanList functions are called.

\section{Value}

An object of class lavaanList, for which several methods are available, including a summary method.

\section{See Also}

class lavaanList

\section{Examples}

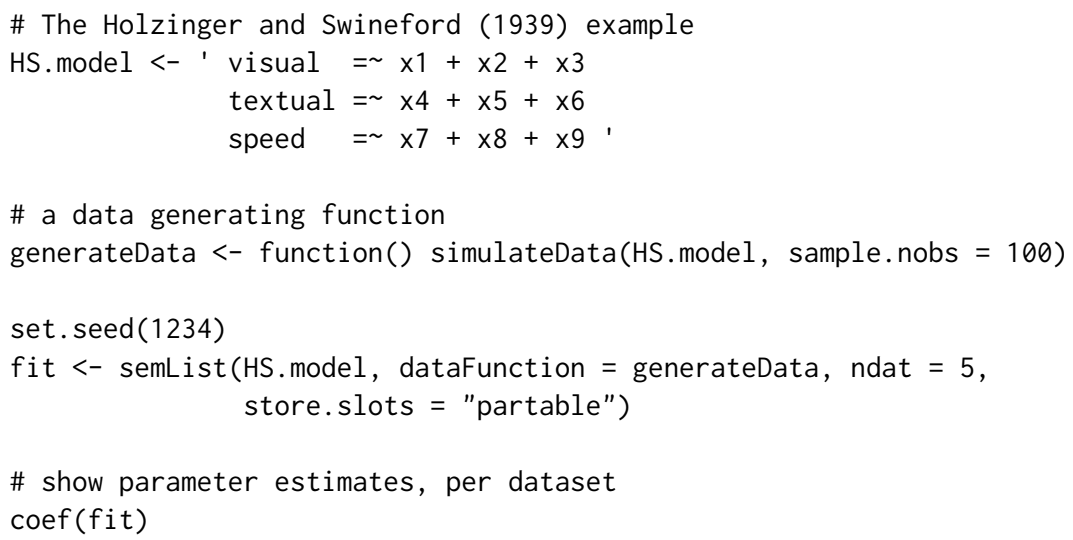




\section{Description}

The lavaanList class represents a collection of (fitted) latent variable models, for a (potentially large) number of datasets. It contains information about the model (which is always the same), and for every dataset a set of (user-specified) slots from a regular lavaan object.

\section{Objects from the Class}

Objects can be created via the cfaList, semList, or lavaanList functions.

\section{Slots}

call: The function call as returned by match. call().

Options: Named list of options that were provided by the user, or filled-in automatically.

ParTable: Named list describing the model parameters. Can be coerced to a data.frame. In the documentation, this is called the 'parameter table'.

pta: Named list containing parameter table attributes.

Data: Object of internal class "Data": information about the data.

Model: Object of internal class "Model": the internal (matrix) representation of the model

meta: List containing additional flags. For internal use only.

timingList: List. Timing slot per dataset.

ParTableList: List. ParTable slot per dataset.

DataList: List. Data slot per dataset.

SampleStatsList: List. SampleStats slot per dataset.

CacheList: List. Cache slot per dataset.

vcovList: List. vcov slot per dataset.

testList: List. test slot per dataset.

optimList: List. optim slot per dataset.

impliedList: List. implied slot per dataset.

h1List: List. h1 slot per dataset.

loglikList: List. loglik slot per dataset.

baselineList: List. baseline slot per dataset.

funList: List. fun slot per dataset.

internalList: List. internal slot per dataset.

external: List. Empty slot to be used by add-on packages. 
Methods

coef signature (object $=$ "lavaanList", type $=$ "free"): Returns the estimates of the parameters in the model as the columns in a matrix; each column corresponds to a different dataset. If type $=$ "free", only the free parameters are returned. If type="user", all parameters listed in the parameter table are returned, including constrained and fixed parameters.

summary signature $($ object $=$ "lavaanList", header $=$ TRUE, estimates $=$ TRUE, nd $=3 \mathrm{~L}):$ Print a summary of the collection of fitted models. If header = TRUE, the header section is printed. If estimates = TRUE, print the parameter estimates section. The argument nd determines the number of digits after the decimal point to be printed (currently only in the parameter estimates section.) Nothing is returned (use parameterEstimates or another extractor function to extract information from this object).

\section{See Also}

cfaList, semList, lavaanList

lavCor

Polychoric, polyserial and Pearson correlations

\section{Description}

Fit an unrestricted model to compute polychoric, polyserial and/or Pearson correlations.

Usage

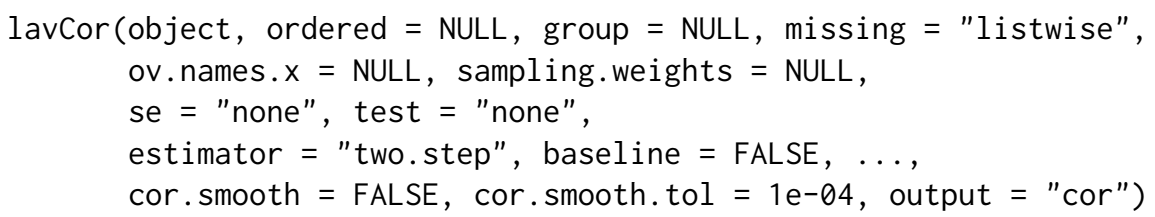

\section{Arguments}

object

ordered

group

missing
Either a data. frame, or an object of class lavaan. If the input is a data. frame, and some variables are declared as ordered factors, lavaan will treat them as ordinal variables.

Character vector. Only used if object is a data. frame. Treat these variables as ordered (ordinal) variables. Importantly, all other variables will be treated as numeric (unless they are declared as ordered in the original data frame.)

Only used if object is a data. frame. Specify a grouping variable.

If "listwise", cases with missing values are removed listwise from the data frame. If "direct" or "ml" or "fiml" and the estimator is maximum likelihood, an EM algorithm is used to estimate the unrestricted covariance matrix (and mean vector). If "pairwise", pairwise deletion is used. If "default", the value is set depending on the estimator and the mimic option. 
sampling. weights

Only used if object is a data.frame. Specify a variable containing sampling weights.

ov.names.x Only used if object is a data.frame. Specify variables that need to be treated as exogenous. Only used if at least one variable is declared as ordered.

se

test

estimator

baseline

$\cdots$

cor.smooth
Only used if output (see below) contains standard errors. See lavOptions for possible options.

Only used if output is "fit" or "lavaan". See lav0ptions for possible options. If "none" or "two.step" or "two.stage", only starting values are computed for the correlations (and thresholds), without any further estimation. If all variables are continuous, the starting values are the sample covariances (converted to correlations if output $=$ "cor"). If at least one variable is ordered, the thresholds are computed using univariate information only. The polychoric and/or polyserial correlations are computed in a second stage, keeping the values of the thresholds constant. If an estimator (other than "two.step" or "two.stage") is specified (for example estimator = "PML"), these starting values are further updated by fitting the unrestricted model using the chosen estimator. See the lavaan function for alternative estimators.

Only used if output is "fit" or "lavaan". If TRUE, a baseline model is also estimated. Note that the test argument should also be set to a value other than "none".

Optional parameters that are passed to the lavaan function.

Logical. Only used if output $=$ "cor". If TRUE, ensure the resulting correlation matrix is positive definite. The following simple method is used: an eigenvalue decomposition is computed; then, eigenvalues smaller than cor.smooth.tol are set to be equal to cor. smooth. tol, before the matrix is again reconstructed. Finally, the matrix (which may no longer have unit diagonal elements) is converted to a correlation matrix using cov2cor.

cor.smooth.tol Numeric. Smallest eigenvalue used when reconstructing the correlation matrix after an eigenvalue decomposition.

output If "cor", the function returns the correlation matrix only. If "cov", the function returns the covariance matrix (this only makes a difference if at least one variable is numeric). If "th" or "thresholds", only the thresholds are returned. If "sampstat", the output equals the result of lavInspect (fit, "sampstat") where fit is the unrestricted model. If "est" or "pe" or "parameterEstimates", the output equals the result of parameterEstimates(fit). Finally, if output is "fit" or "lavaan", the function returns an object of class lavaan.

\section{Details}

This function is a wrapper around the lavaan function, but where the model is defined as the unrestricted model. The following free parameters are included: all covariances/correlations among the variables, variances for continuous variables, means for continuous variables, thresholds for ordered variables, and if exogenous variables are included (ov.names. $x$ is not empty) while some variables are ordered, also the regression slopes enter the model. 


\section{Value}

By default, if output = "cor" or output = "cov", a symmetric matrix (of class "lavaan . matrix. symmetric", which only affects the way the matrix is printed). If output = "th", a named vector of thresholds. If output = "fit" or output = "lavaan", an object of class lavaan.

\section{References}

Olsson, U. (1979). Maximum likelihood estimation of the polychoric correlation coefficient. Psychometrika, 44(4), 443-460.

Olsson, U., Drasgow, F., \& Dorans, N. J. (1982). The polyserial correlation coefficient. Psychometrika, 47(3), 337-347.

\section{See Also}

lavaan

\section{Examples}

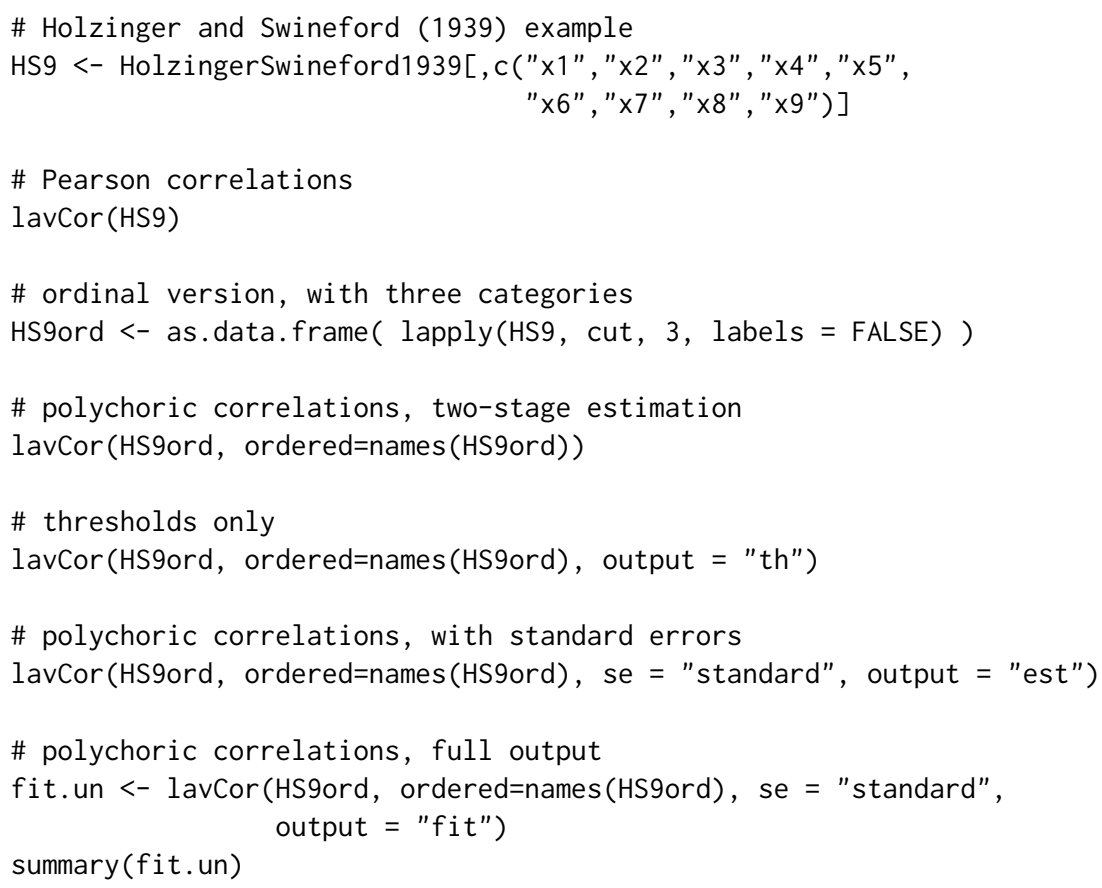

\section{Description}

Export a fitted lavaan object to an external program. 
Usage

lavExport(object, target $=$ "lavaan", prefix $=$ "sem", dir.name $=$ "lavExport", export $=$ TRUE)

\section{Arguments}
object
An object of class lavaan.
target
The target program. Current options are "lavaan" and "Mplus".
prefix
The prefix used to create the input files; the name of the input file has the pattern 'prefix dot target dot in'; the name of the data file has the pattern 'prefix dot target dot raw'.
dir.name
The directory name (including a full path) where the input files will be written.
export
If TRUE, the files are written to the output directory (dir. name). If FALSE, only the syntax is generated as a character string.

\section{Details}

This function was mainly created to quickly generate an Mplus syntax file to compare the results between Mplus and lavaan. The target "lavaan" can be useful to create a full model syntax as needed for the lavaan() function. More targets (perhaps for LISREL or EQS) will be added in future releases.

\section{Value}

If export $=$ TRUE, a directory (called lavExport by default) will be created, typically containing a data file, and an input file so that the same analysis can be run using an external program. If export = FALSE, a character string containing the model syntax only for the target program.

\section{See Also}

lavaanify, mplus2lavaan

\section{Examples}

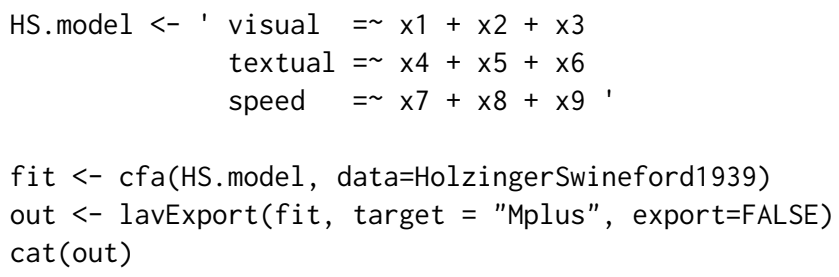


lavInspect Inspect or extract information from a fitted lavaan object

\section{Description}

The lavInspect() and lavTech() functions can be used to inspect/extract information that is stored inside (or can be computed from) a fitted lavaan object. Note: the (older) inspect () function is now simply a shortcut for lavInspect() with default arguments.

Usage

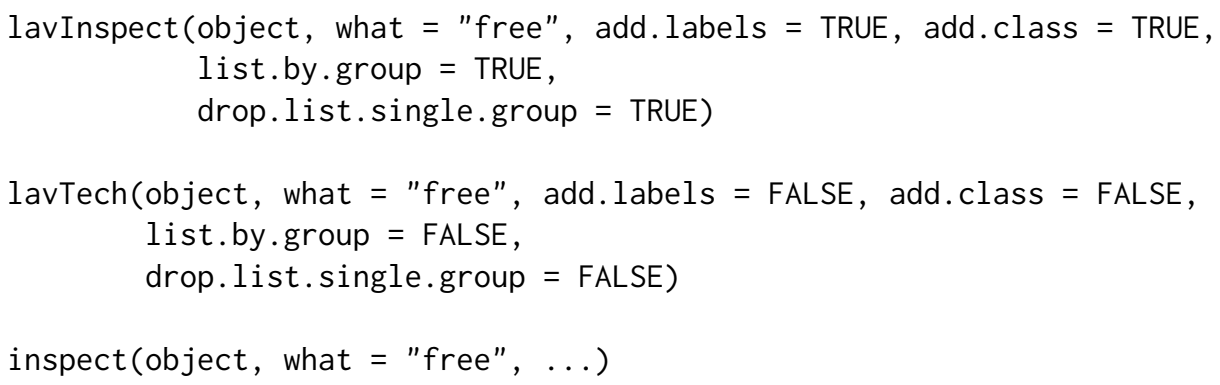

\section{Arguments}

object

An object of class lavaan.

what

Character. What needs to be inspected/extracted? See Details for a full list. Note: the what argument is not case-sensitive (everything is converted to lower case.)

add. labels If TRUE, variable names are added to the vectors and/or matrices.

add.class If TRUE, vectors are given the 'lavaan.vector' class; matrices are given the 'lavaan.matrix' class, and symmetric matrices are given the 'lavaan.matrix.symmetric' class. This only affects the way they are printed on the screen.

list.by.group Logical. Only used when the output are model matrices. If TRUE, the model matrices are nested within groups. If FALSE, a flattened list is returned containing all model matrices, with repeated names for multiple groups.

drop.list.single.group

If FALSE, the results are returned as a list, where each element corresponds to a group (even if there is only a single group). If TRUE, the list will be unlisted if there is only a single group.

... Additional arguments. Not used by lavaan, but by other packages.

\section{Details}

The lavInspect() and lavTech() functions only differ in the way they return the results. The lavInspect() function will prettify the output by default, while the lavTech() will not attempt to prettify the output by default. The (older) inspect () function is a simplified version of lavInspect() with only the first two arguments. 
Below is a list of possible values for the what argument, organized in several sections:

Model matrices:

"free": A list of model matrices. The non-zero integers represent the free parameters. The numbers themselves correspond to the position of the free parameter in the parameter vector. This determines the order of the model parameters in the output of for example coef () and $v \operatorname{cov}()$.

"partable": A list of model matrices. The non-zero integers represent both the fixed parameters (for example, factor loadings fixed at 1.0), and the free parameters if we ignore any equality constraints. They correspond with all entries (fixed or free) in the parameter table. See parTable.

"se": A list of model matrices. The non-zero numbers represent the standard errors for the free parameters in the model. If two parameters are constrained to be equal, they will have the same standard error for both parameters. Aliases: "std.err" and "standard.errors".

"start": A list of model matrices. The values represent the starting values for all model parameters. Alias: "starting.values".

"est": A list of model matrices. The values represent the estimated model parameters. Aliases: "estimates", and " $x$ ".

"dx. free": A list of model matrices. The values represent the gradient (first derivative) values of the model parameters. If two parameters are constrained to be equal, they will have the same gradient value.

"dx.all": A list of model matrices. The values represent the first derivative with respect to all possible matrix elements. Currently, this is only available when the estimator is "ML" or "GLS".

"std": A list of model matrices. The values represent the (completely) standardized model parameters (the variances of both the observed and the latent variables are set to unity). Aliases: "std.all", "standardized".

"std.lv": A list of model matrices. The values represent the standardized model parameters (only the variances of the latent variables are set to unity.)

"std.nox": A list of model matrices. The values represent the (completely) standardized model parameters (the variances of both the observed and the latent variables are set to unity; however, the variances of any observed exogenous variables are not set to unity; hence no-x.)

Information about the data:

"data": A matrix containing the observed variables that have been used to fit the model. No column/row names are provided. Column names correspond to the output of lavNames (object), while the rows correspond to the output of lavInspect (object, "case.idx".

"ordered": A character vector. The ordered variables.

"nobs": Integer vector. The number of observations in each group that were used in the analysis.

"norig": Integer vector. The original number of observations in each group.

"ntotal": Integer. The total number of observations that were used in the analysis. If there is just a single group, this is the same as the "nobs" option; if there are multiple groups, this is the sum of the "nobs" numbers for each group.

"case.idx": Integer vector. The case/observation numbers that were used in the analysis. In the case of multiple groups: a list of numbers. 
"empty.idx": The case/observation numbers of those cases/observations that contained missing values only (at least for the observed variables that were included in the model). In the case of multiple groups: a list of numbers.

"patterns": A binary matrix. The rows of the matrix are the missing data patterns where 1 and 0 denote non-missing and missing values for the corresponding observed variables respectively (or TRUE and FALSE if lavTech() is used.) If the data is complete (no missing values), there will be only a single pattern. In the case of multiple groups: a list of pattern matrices.

"coverage": A symmetric matrix where each element contains the proportion of observed datapoints for the corresponding pair of observed variables. In the case of multiple groups: a list of coverage matrices.

"group": A character string. The group variable in the data.frame (if any).

"ngroups": Integer. The number of groups.

"group. label": A character vector. The group labels.

"level.label": A character vector. The level labels.

"cluster": A character vector. The cluster variable(s) in the data.frame (if any).

"nlevels": Integer. The number of levels.

"nclusters": Integer. The number of clusters that were used in the analysis.

"ncluster. size": Integer. The number of different cluster sizes.

"cluster.size": Integer vector. The number of observations within each cluster. For multigroup multilevel models, a list of integer vectors, indicating cluster sizes within each group.

"cluster.id": Integer vector. The cluster IDs identifying the clusters. For multigroup multilevel models, a list of integer vectors, indicating cluster IDs within each group.

"cluster.idx": Integer vector. The cluster index for each observation. The cluster index ranges from 1 to the number of clusters. For multigroup multilevel models, a list of integer vectors, indicating cluster indices within each group.

"cluster. label": Integer vector. The cluster ID for each observation. For multigroup multilevel models, a list of integer vectors, indicating the cluster ID for each observation within each group.

"cluster.sizes": Integer vector. The different cluster sizes that were used in the analysis. For multigroup multilevel models, a list of integer vectors, indicating the different cluster sizes within each group.

"average.cluster. size": Integer. The average cluster size (using the formula $s=\left(N^{\wedge} 2-\operatorname{sum}(\right.$ cluster. size^2)) $/(\mathrm{N} *(\mathrm{ncluster} \mathrm{-}-1 \mathrm{~L})))$. For multigroup multilevel models, a list containing the average cluster size per group.

Observed sample statistics:

"sampstat": Observed sample statistics. Aliases: "obs", "observed", "samp", "sample", "samplestatistics". Since 0.6-3, we always check if an h1 slot is available (the estimates for the unrestricted model); if present, we extract the sample statistics from this slot. This implies that if variables are continuous, and missing $=$ "ml" (or "fiml"), we return the covariance matrix (and mean vector) as computed by the EM algorithm under the unrestricted (h1) model. If the h1 is not present (perhaps, because the model was fitted with h1 = FALSE), we return the sample statistics from the SampleStats slot. Here, pairwise deletion is used for the elements of the covariance matrix (or correlation matrix), and listwise deletion for all univariate statistics (means, intercepts and thresholds). 
"sampstat.h1": Deprecated. Do not use any longer.

"wls.obs": The observed sample statistics (covariance elements, intercepts/thresholds, etc.) in a single vector.

"wls.v": The weight vector as used in weighted least squares estimation.

"gamma": N times the asymptotic variance matrix of the sample statistics. Alias: "sampstat.nacov".

Model features:

"meanstructure": Logical. TRUE if a meanstructure was included in the model.

"categorical": Logical. TRUE if categorical endogenous variables were part of the model.

"fixed.x": Logical. TRUE if the exogenous x-covariates are treated as fixed.

"parameterization": Character. Either "delta" or "theta".

Model-implied sample statistics:

"implied": The model-implied summary statistics. Alias: "fitted", "expected", "exp".

"resid": The difference between observed and model-implied summary statistics. Alias: "residuals", "residual", "res".

"cov.lv": The model-implied variance-covariance matrix of the latent variables. Alias: "veta" [for $\mathrm{V}(\mathrm{eta})]$.

"cor.lv": The model-implied correlation matrix of the latent variables.

"mean. lv": The model-implied mean vector of the latent variables. Alias: "eeta" [for E(eta)].

"cov.ov": The model-implied variance-covariance matrix of the observed variables. Aliases: "sigma", "sigma.hat".

"cor.ov": The model-implied correlation matrix of the observed variables.

"mean.ov": The model-implied mean vector of the observed variables. Aliases: "mu", "mu. hat".

"cov.all": The model-implied variance-covariance matrix of both the observed and latent variables.

"cor.all": The model-implied correlation matrix of both the observed and latent variables.

"th": The model-implied thresholds. Alias: "thresholds".

"wls.est": The model-implied sample statistics (covariance elements, intercepts/thresholds, etc.) in a single vector.

"vy": The model-implied unconditional variances of the observed variables.

"rsquare": The R-square value for all endogenous variables. Aliases: "r-square", "r2".

Optimizer information:

"converged": Logical. TRUE if the optimizer has converged; FALSE otherwise.

"iteratons": Integer. The number of iterations used by the optimizer.

"optim": List. All available information regarding the optimization results.

"npar": Integer. Number of free parameters (ignoring constraints).

"coef": Numeric. The estimated parameter vector. 
Gradient, Hessian, observed, expected and first.order information matrices:

"gradient": Numeric vector containing the first derivatives of the discrepancy function with respect to the (free) model parameters.

"hessian": Matrix containing the second derivatives of the discrepancy function with respect to the (free) model parameters.

"information": Matrix containing either the observed or the expected information matrix (depending on the information option of the fitted model). This is unit-information, not totalinformation.

"information. expected": Matrix containing the expected information matrix for the free model parameters.

"information.observed": Matrix containing the observed information matrix for the free model parameters.

"information.first.order": Matrix containing the first.order information matrix for the free model parameters. This is the outer product of the gradient elements (the first derivative of the discrepancy function with respect to the (free) model parameters). Alias: "first.order".

"augmented. information": Matrix containing either the observed or the expected augmented (or bordered) information matrix (depending on the information option of the fitted model. Only relevant if constraints have been used in the model.

"augmented.information. expected": Matrix containing the expected augmented (or bordered) information matrix. Only relevant if constraints have been used in the model.

"augmented.information. observed": Matrix containing the observed augmented (or bordered) information matrix. Only relevant if constraints have been used in the model.

"augmented.information.first.order": Matrix containing the first.order augmented (or bordered) information matrix. Only relevant if constraints have been used in the model.

"inverted.information": Matrix containing either the observed or the expected inverted information matrix (depending on the information option of the fitted model.

"inverted.information.expected": Matrix containing the inverted expected information matrix for the free model parameters.

"inverted.information.observed": Matrix containing the inverted observed information matrix for the free model parameters.

"inverted.information.first.order": Matrix containing the inverted first.order information matrix for the free model parameters.

"h1. information": Matrix containing either the observed, expected or first.order information matrix (depending on the information option of the fitted model) of the unrestricted h1 model. This is unit-information, not total-information.

"h1.information.expected": Matrix containing the expected information matrix for the unrestricted h1 model.

"h1. information. observed": Matrix containing the observed information matrix for the unrestricted h1 model.

"h1. information. first.order": Matrix containing the first.order information matrix for the the unrestricted h1 model. Alias: "h1.first.order".

Variance covariance matrix of the model parameters: 
"vcov": Matrix containing the variance covariance matrix of the estimated model parameters.

"vcov.std.all": Matrix containing the variance covariance matrix of the standardized estimated model parameters. Standardization is done with respect to both observed and latent variables.

"vcov.std.1v": Matrix containing the variance covariance matrix of the standardized estimated model parameters. Standardization is done with respect to the latent variables only.

"vcov.std.nox": Matrix containing the variance covariance matrix of the standardized estimated model parameters. Standardization is done with respect to both observed and latent variables, but ignoring any exogenous observed covariates.

"vcov.def": Matrix containing the variance covariance matrix of the user-defined (using the := operator) parameters.

"vcov.def.std.all": Matrix containing the variance covariance matrix of the standardized userdefined parameters. Standardization is done with respect to both observed and latent variables.

"vcov.def.std.lv": Matrix containing the variance covariance matrix of the standardized userdefined parameters. Standardization is done with respect to the latent variables only.

"vcov. def.std.nox": Matrix containing the variance covariance matrix of the standardized userdefined parameters. Standardization is done with respect to both observed and latent variables, but ignoring any exogenous observed covariates.

"vcov. def. joint": Matrix containing the joint variance covariance matrix of both the estimated model parameters and the defined (using the $:=$ operator) parameters.

"vcov.def.joint.std.all": Matrix containing the joint variance covariance matrix of both the standardized model parameters and the user-defined parameters. Standardization is done with respect to both observed and latent variables.

"vcov.def.joint.std.lv": Matrix containing the joint variance covariance matrix of both the standardized model parameters and the user-defined parameters. Standardization is done with respect to the latent variables only.

"vcov.def.joint.std.nox": Matrix containing the joint variance covariance matrix of both the standardized model parameters and the user-defined parameters. Standardization is done with respect to both observed and latent variables, but ignoring any exogenous observed covariates.

Miscellaneous:

"coef. boot": Matrix containing estimated model parameters for for each bootstrap sample. Only relevant when bootstrapping was used.

"UGamma": Matrix containing the product of ' $U$ ' and 'Gamma' matrices as used by the SatorraBentler correction. The trace of this matrix, divided by the degrees of freedom, gives the scaling factor.

"UfromUGamma": Matrix containing the 'U' matrix as used by the Satorra-Bentler correction. Alias: "U".

"list": The parameter table. The same output as given by parTable().

"fit": The fit measures. Aliases: "fitmeasures", "fit.measures", "fit.indices". The same output as given by fitMeasures().

"mi": The modification indices. Alias: "modindices", "modification.indices". The same output as given by modindices(). 
"loglik. casewise": Vector containing the casewise loglikelihood contributions. Only available if estimator $=$ "ML".

"options": List. The option list.

"call": List. The call as returned by match.call, coerced to a list.

"timing": List. The timing (in milliseconds) of various lavaan subprocedures.

"test": List. All available information regarding the (goodness-of-fit) test statistic(s).

"baseline.test": List. All available information regarding the (goodness-of-fit) test statistic(s) of the baseline model.

"baseline.partable": Data.frame. The parameter table of the (internal) baseline model.

"post. check": Post-fitting check if the solution is admissible. A warning is raised if negative variances are found, or if either lavInspect (fit, "cov.lv") or lavInspect(fit, "theta") return a non-positive definite matrix.

"zero.cell.tables": List. List of bivariate frequency tables where at least one cell is empty.

"version": The lavaan version number that was used to construct the fitted lavaan object.

\section{See Also}

lavaan

\section{Examples}

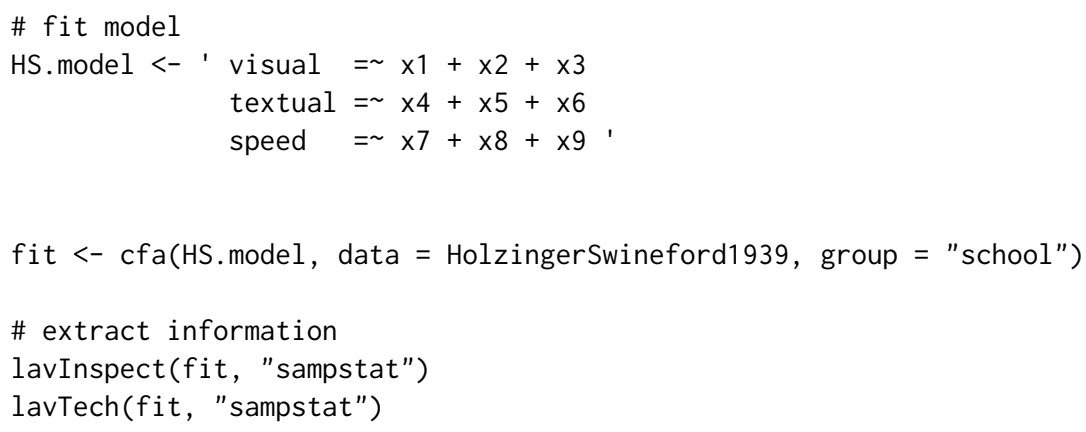

lavListInspect

Inspect or extract information from a lavaanList object

\section{Description}

The lavListInspect() and lavListTech() functions can be used to inspect/extract information that is stored inside (or can be computed from) a lavaanList object. 


\section{Usage}

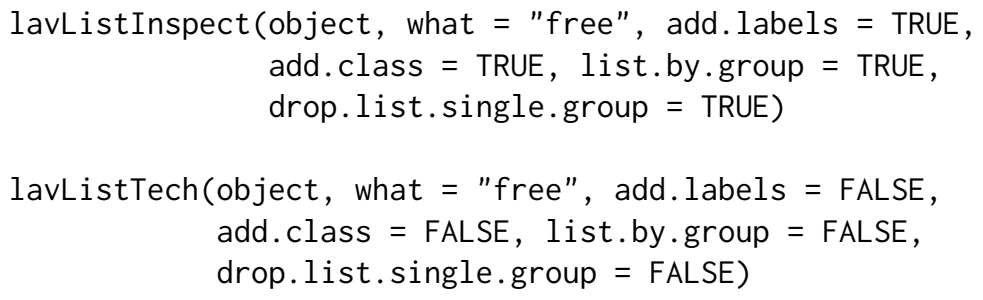

\section{Arguments}

object

what

An object of class lavaanList.

Character. What needs to be inspected/extracted? See Details for a full list. Note: the what argument is not case-sensitive (everything is converted to lower case.)

add. labels If TRUE, variable names are added to the vectors and/or matrices.

add.class If TRUE, vectors are given the 'lavaan.vector' class; matrices are given the 'lavaan.matrix' class, and symmetric matrices are given the 'lavaan.matrix.symmetric' class. This only affects the way they are printed on the screen.

list.by. group Logical. Only used when the output are model matrices. If TRUE, the model matrices are nested within groups. If FALSE, a flattened list is returned containing all model matrices, with repeated names for multiple groups.

drop.list.single.group

If FALSE, the results are returned as a list, where each element corresponds to a group (even if there is only a single group.) If TRUE, the list will be unlisted if there is only a single group.

\section{Details}

The lavListInspect() and lavListTech() functions only differ in the way they return the results. The lavListInspect() function will prettify the output by default, while the lavListTech() will not attempt to prettify the output by default.

Below is a list of possible values for the what argument, organized in several sections:

Model matrices:

"free": A list of model matrices. The non-zero integers represent the free parameters. The numbers themselves correspond to the position of the free parameter in the parameter vector. This determines the order of the model parameters in the output of for example coef () and $v \operatorname{cov}()$.

"partable": A list of model matrices. The non-zero integers represent both the fixed parameters (for example, factor loadings fixed at 1.0), and the free parameters if we ignore any equality constraints. They correspond with all entries (fixed or free) in the parameter table. See parTable.

"start": A list of model matrices. The values represent the starting values for all model parameters. Alias: "starting.values".

Information about the data (including missing patterns): 
"group": A character string. The group variable in the data.frame (if any).

"ngroups": Integer. The number of groups.

"group. label": A character vector. The group labels.

"level. label": A character vector. The level labels.

"cluster": A character vector. The cluster variable(s) in the data.frame (if any).

"nlevels": Integer. The number of levels.

"ordered": A character vector. The ordered variables.

"nobs": Integer vector. The number of observations in each group that were used in the analysis (in each dataset).

"norig": Integer vector. The original number of observations in each group (in each dataset).

"ntotal": Integer. The total number of observations that were used in the analysis. If there is just a single group, this is the same as the "nobs" option; if there are multiple groups, this is the sum of the "nobs" numbers for each group (in each dataset).

Model features:

"meanstructure": Logical. TRUE if a meanstructure was included in the model.

"categorical": Logical. TRUE if categorical endogenous variables were part of the model.

"fixed.x": Logical. TRUE if the exogenous x-covariates are treated as fixed.

"parameterization": Character. Either "delta" or "theta".

"list": The parameter table. The same output as given by parTable().

"options": List. The option list.

"call": List. The call as returned by match.call, coerced to a list.

\section{See Also}

lavaanList

\section{Examples}

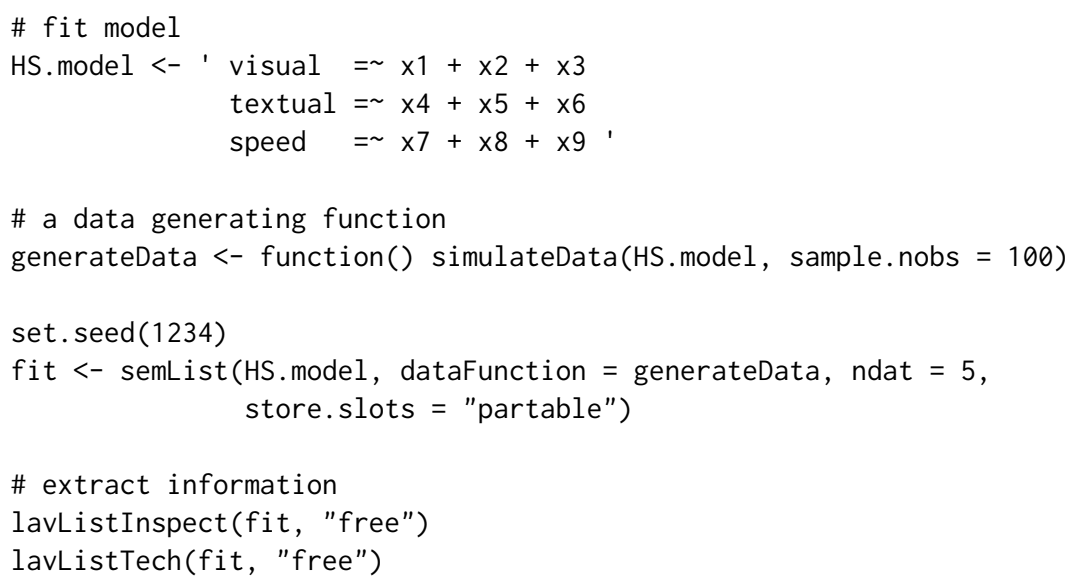


lavMatrixRepresentation

lavaan matrix representation

\section{Description}

Extend the parameter table with a matrix representation.

\section{Usage}

lavMatrixRepresentation (partable, representation = "LISREL", add. attributes = FALSE, as.data.frame. $=$ TRUE)

\section{Arguments}

partable A lavaan parameter table (as extracted by the parTable function, or generated by the lavPartable function).

representation Character. The matrix representation style. Currently, only the all-y version of the LISREL representation is supported.

add.attributes Logical. If TRUE, additional information about the model matrix representation is added as attributes.

as . data. frame. Logical. If TRUE, the extended parameter table is returned as a data.frame.

\section{Value}

A list or a data.frame containing the original parameter table, plus three columns: a "mat" column containing matrix names, and a "row" and "col" column for the row and column indices of the model parameters in the model matrices.

\section{See Also}

lavParTable, parTable

\section{Examples}

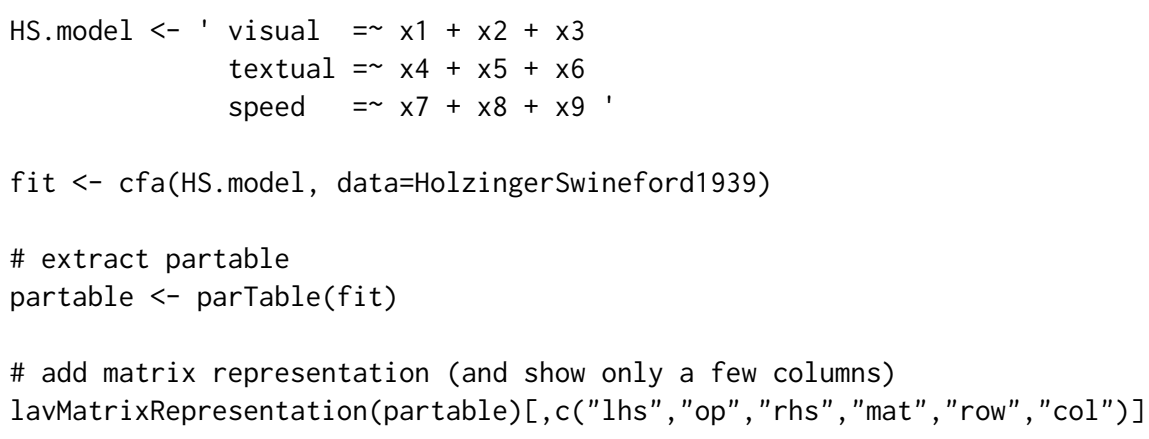




lavNames lavaan Names

\section{Description}

Extract variables names from a fitted lavaan object.

\section{Usage}

lavNames (object, type $=$ "ov", $\ldots$ )

\section{Arguments}

object

type
An object of class lavaan.

Character. The type of variables whose names should be extracted. See details for a complete list.

Additional selection variables. For example "group $=2 \mathrm{~L}$ " (in a multiple-group analysis) only considers the variables included in the model for the second group.

\section{Details}

The order of the variable names, as returned by lavNames determines the order in which the variables are listed in the parameter table, and therefore also in the summary output.

The following variable types are available:

- "ov": observed variables

- "ov.x": (pure) exogenous observed variables (no mediators)

- "ov.nox": non-exogenous observed variables

- "ov.model": modelled observed variables (joint vs conditional)

- "ov.y": (pure) endogenous variables (dependent only) (no mediators)

- "ov. num": numeric observed variables

- "ov.ord": ordinal observed variables

- "ov.ind": observed indicators of latent variables

- "ov.orphan": lonely observed variables (only intercepts/variancesappear in the model syn$\operatorname{tax})$

- "ov. interaction": interaction terms (defined by the colon operator)

- "th": threshold names ordinal variables only

- "th.mean": threshold names ordinal + numeric variables (if any)

- "lv": latent variables

- "lv. regular": latent variables (defined by $=\sim$ only)

- "lv. formative": latent variables (defined by < only) 
- "lv.x": (pure) exogenous variables

- "lv.y": (pure) endogenous variables

- "lv.nox": non-exogenous latent variables

- "lv.nonnormal": latent variables with non-normal indicators

- "lv.interaction": interaction terms at the latent level

- "eqs.y": variables that appear as dependent variables in a regression formula (but not indicators of latent variables)

- "eqs. $x "$ : variables that appear as independent variables in a regression formula

\section{See Also}

lavaanify, parTable

\section{Examples}

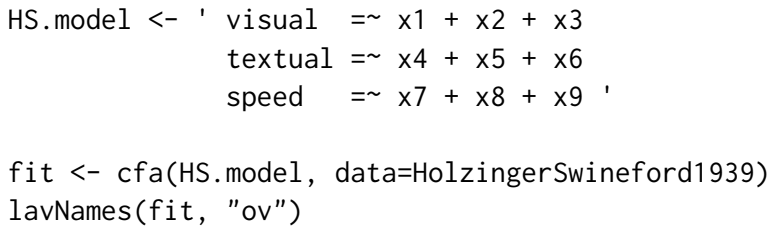

lavOptions $\quad$ lavaan Options

\section{Description}

Show the default options used by the lavaan() function. The options can be changed by passing 'name = value' arguments to the lavaan() function call, where they will be added to the '...' argument.

\section{Usage}

lavOptions $(x=$ NULL, default $=$ NULL, mimic $=$ "lavaan" $)$

\section{Arguments}

$x$

default

mimic
Character. A character string holding an option name, or a character string vector holding multiple option names. All option names are converted to lower case.

If a single option is specified but not available, this value is returned.

Character. Adjust the option list for this mimic flag. 


\section{Details}

This is the full list of options that are accepted by the lavaan() function, organized in several sections:

Model features (always available):

meanstructure: If TRUE, the means of the observed variables enter the model. If "default", the value is set based on the user-specified model, and/or the values of other arguments.

int.ov. free: If FALSE, the intercepts of the observed variables are fixed to zero.

int.lv. free: If FALSE, the intercepts of the latent variables are fixed to zero.

conditional.x: If TRUE, we set up the model conditional on the exogenous ' $x$ ' covariates; the model-implied sample statistics only include the non- $x$ variables. If FALSE, the exogenous ' $x$ ' variables are modeled jointly with the other variables, and the model-implied statistics refect both sets of variables. If "default", the value is set depending on the estimator, and whether or not the model involves categorical endogenous variables.

fixed. $x$ : If TRUE, the exogenous ' $x$ ' covariates are considered fixed variables and the means, variances and covariances of these variables are fixed to their sample values. If FALSE, they are considered random, and the means, variances and covariances are free parameters. If "default", the value is set depending on the mimic option.

or thogonal: If TRUE, all covariances among latent variables are set to zero.

or thogonal.y: If TRUE, all covariances among endogenous latent variables only are set to zero.

or thogonal.x: If TRUE, all covariances among exogenous latent variables only are set to zero.

std. lv: If TRUE, the metric of each latent variable is determined by fixing their (residual) variances to 1.0. If FALSE, the metric of each latent variable is determined by fixing the factor loading of the first indicator to 1.0. If there are multiple groups, std.lv = TRUE and "loadings" is included in the group. equal argument, then only the latent variances of the first group will be fixed to 1.0, while the latent variances of other groups are set free.

effect.coding: Can be logical or character string. If logical and TRUE, this implies effect. coding $=c$ ("loadings", "intercepts"). If logical and FALSE, it is set equal to the empty string. If "loadings" is included, equality constraints are used so that the average of the factor loadings (per latent variable) equals 1 . Note that this should not be used together with std. lv = TRUE. If "intercepts" is included, equality constraints are used so that the sum of the intercepts (belonging to the indicators of a single latent variable) equals zero. As a result, the latent mean will be freely estimated and usually equal the average of the means of the involved indicators.

ceq. simple: Logical. If TRUE, and no other general (equality or inequality) constraints are used in the model, simple equality constraints are represented in the parameter table as duplicated free parameters (instead of extra rows with op $="=="$ ).

parameterization: Currently only used if data is categorical. If "delta", the delta parameterization is used. If "theta", the theta parameterization is used.

Model features (only available for the lavaan() function):

auto.fix.first: If TRUE, the factor loading of the first indicator is set to 1.0 for every latent variable.

auto.fix.single: If TRUE, the residual variance (if included) of an observed indicator is set to zero if it is the only indicator of a latent variable. 
auto.var: If TRUE, the residual variances and the variances of exogenous latent variables are included in the model and set free.

auto.cov.lv.x: If TRUE, the covariances of exogenous latent variables are included in the model and set free.

auto.cov.y: If TRUE, the covariances of dependent variables (both observed and latent) are included in the model and set free.

auto. th: If TRUE, thresholds for limited dependent variables are included in the model and set free.

auto.delta: If TRUE, response scaling parameters for limited dependent variables are included in the model and set free.

auto. efa: If TRUE, the necessary constraints are imposed to make the (unrotated) exploratory factor analysis blocks identifiable: for each block, factor variances are set to 1 , factor covariances are constrained to be zero, and factor loadings are constrained to follow an echelon pattern.

Data options:

std.ov: If TRUE, all observed variables are standardized before entering the analysis.

missing: The default setting is "listwise": all cases with missing values are removed listwise from the data before the analysis starts. This is only valid if the data are missing completely at random (MCAR). Therefore, it may not be the optimal choice, but it can be useful for a first run. If the estimator belongs to the ML family, another option is "ml" (alias: "fiml" or "direct"). This corresponds to the so-called full information maximum likelihood approach (fiml), where we compute the likelihood case by case, using all available data from that case. Note that if the model contains exogenous observed covariates, and fixed. $x=$ TRUE (the default), all cases with any missing values on these covariates will be deleted first. The option "ml.x" (alias: "fiml. $x$ " or "direct. $x "$ ") is similar to "ml", but does not delete any cases with missing values for the exogenous covariates, even if fixed. $x=$ TRUE. (Note: all lavaan versions $<0.6$ used " $m l . x$ " instead of " $m l$ "). If you wish to use multiple imputation, you need to use an external package (eg. mice) to generate imputed datasets, which can then be analyzed using the semList function. The semTools package contains several functions to do this automatically. Another option (with continuous data) is to use "two.stage" or "robust. two.stage". In this approach, we first estimate the sample statistics (mean vector, variance-covariance matrix) using an EM algorithm. Then, we use these estimated sample statistics as input for a regular analysis (as if the data were complete). The standard errors and test statistics are adjusted correctly to reflect the two-step procedure. The "robust. two.stage" option produces standard errors and a test statistic that are robust against non-normality. If (part of) the data is categorical, and the estimator is from the (W)LS family, the only option (besides listwise deletion) is "pairwise". In this three-step approach, missingness is only an issue in the first two steps. In the first step, we compute thresholds (for categorical variables) and means or intercepts (for continuous variables) using univariate information only. In this step, we simply ignore the missing values just like in mean(x, na.rm = TRUE). In the second step, we compute polychoric/polyserial/pearson correlations using (only) two variables at a time. Here we use pairwise deletion: we only keep those observations for which both values are observed (not-missing). And this may change from pair to pair. By default, in the categorical case we use conditional. $x=$ TRUE. Therefore, any cases with missing values on the exogenous covariates will be deleted listwise from the data first. Finally, if the estimator is "PML", the available options are "pairwise", "available.cases" and "doubly.robust". See the PML tutorial on the lavaan website for more information about these approaches. 
sampling.weights.normalization: If "none", the sampling weights (if provided) will not be transformed. If "total", the sampling weights are normalized by dividing by the total sum of the weights, and multiplying again by the total sample size. If "group", the sampling weights are normalized per group: by dividing by the sum of the weights (in each group), and multiplying again by the group size. The default is "total".

Data summary options:

sample.cov.rescale: If TRUE, the sample covariance matrix provided by the user is internally rescaled by multiplying it with a factor $(\mathrm{N}-1) / \mathrm{N}$. If "default", the value is set depending on the estimator and the likelihood option: it is set to TRUE if maximum likelihood estimation is used and likelihood="normal", and FALSE otherwise.

ridge: Numeric. Small constant used for ridging. Only used if the sample covariance matrix is non positive definite.

Multiple group options:

group. label: A character vector. The user can specify which group (or factor) levels need to be selected from the grouping variable, and in which order. If missing, all grouping levels are selected, in the order as they appear in the data.

group. equal: A vector of character strings. Only used in a multiple group analysis. Can be one or more of the following: "loadings", "composite.loadings", "intercepts", "means", "thresholds", "regressions", "residuals", "residual.covariances", "lv.variances" or "lv. covariances", specifying the pattern of equality constraints across multiple groups.

group.partial: A vector of character strings containing the labels of the parameters which should be free in all groups (thereby overriding the group.equal argument for some specific parameters).

group.w. free: Logical. If TRUE, the group frequencies are considered to be free parameters in the model. In this case, a Poisson model is fitted to estimate the group frequencies. If FALSE (the default), the group frequencies are fixed to their observed values.

Estimation options:

estimator: The estimator to be used. Can be one of the following: "ML" for maximum likelihood, "GLS" for (normal theory) generalized least squares, "WLS" for weighted least squares (sometimes called ADF estimation), "ULS" for unweighted least squares, "DWLS" for diagonally weighted least squares, and "DLS" for distributionally-weighted least squares. These are the main options that affect the estimation. For convenience, the "ML" option can be extended as "MLM", "MLMV", "MLMVS", "MLF", and "MLR". The estimation will still be plain "ML", but now with robust standard errors and a robust (scaled) test statistic. For "MLM", "MLMV", "MLMVS", classic robust standard errors are used (se="robust. sem"); for "MLF", standard errors are based on first-order derivatives (information = "first.order"); for "MLR", 'HuberWhite' robust standard errors are used (se="robust. huber. white"). In addition, "MLM" will compute a Satorra-Bentler scaled (mean adjusted) test statistic (test="satorra.bentler"), "MLMVS" will compute a mean and variance adjusted test statistic (Satterthwaite style) (test="mean .var .adjusted"), "MLMV" will compute a mean and variance adjusted test statistic (scaled and shifted) (test="scaled. shifted"), and "MLR" will compute a test statistic which is asymptotically equivalent to the Yuan-Bentler T2-star test statistic (test="yuan. bentler.mplus"). Analogously, the estimators "WLSM" 
and "WLSMV" imply the "DWLS" estimator (not the "WLS" estimator) with robust standard errors and a mean or mean and variance adjusted test statistic. Estimators "ULSM" and "ULSMV" imply the "ULS" estimator with robust standard errors and a mean or mean and variance adjusted test statistic.

likelihood: Only relevant for ML estimation. If "wishart", the wishart likelihood approach is used. In this approach, the covariance matrix has been divided by $\mathrm{N}-1$, and both standard errors and test statistics are based on N-1. If "normal", the normal likelihood approach is used. Here, the covariance matrix has been divided by $\mathrm{N}$, and both standard errors and test statistics are based on N. If "default", it depends on the mimic option: if mimic="lavaan" or mimic="Mplus", normal likelihood is used; otherwise, wishart likelihood is used.

link: Not used yet. This is just a placeholder until the MML estimator is back.

information: If "expected", the expected information matrix is used (to compute the standard errors). If "observed", the observed information matrix is used. If "first.order", the information matrix is based on the outer product of the casewise scores. See also the options "h1. information" and "observed.information" for further control. If "default", the value is set depending on the estimator, the missing argument, and the mimic option. If the argument is a vector with two elements, the first element is used for the computation of the standard errors, while the second element is used for the (robust) test statistic.

h1. information: If "structured" (the default), the unrestricted (h1) information part of the (expected, first.order or observed if h1 is used) information matrix is based on the structured, or model-implied statistics (model-implied covariance matrix, model-implied mean vector, etc.). If "unstructured", the unrestricted (h1) information part is based on sample-based statistics (observed covariance matrix, observed mean vector, etc.) If the argument is a vector with two elements, the first element is used for the computation of the standard errors, while the second element is used for the (robust) test statistic.

observed.information: If "hessian", the observed information matrix is based on the hessian of the objective function. If "h1", an approximation is used that is based on the observed information matrix of the unrestricted (h1) model. If the argument is a vector with two elements, the first element is used for the computation of the standard errors, while the second element is used for the (robust) test statistic.

se: If "standard", conventional standard errors are computed based on inverting the (expected, observed or first.order) information matrix. If "robust.sem", conventional robust standard errors are computed. If "robust.huber.white", standard errors are computed based on the 'mlr' (aka pseudo ML, Huber-White) approach. If "robust", either "robust.sem" or "robust.huber.white" is used depending on the estimator, the mimic option, and whether the data are complete or not. If "boot" or "bootstrap", bootstrap standard errors are computed using standard bootstrapping (unless Bollen-Stine bootstrapping is requested for the test statistic; in this case bootstrap standard errors are computed using model-based bootstrapping). If "none", no standard errors are computed.

test: Character vector. See the documentation of the lavTest function for a full list. Multiple names of test statistics can be provided. If "default", the value depends on the values of other arguments. See also the lavTest function to extract (alternative) test statistics from a fitted lavaan object.

codescaled.test: Character. Choose the test statistic that will be scaled (if a scaled test statistic is requested). The default is "standard", but it could also be (for example) "Browne . residual .nt".

gamma.n.minus. one Logical. If TRUE, we divide the Gamma matrix by N-1 (instead of the default $\mathrm{N})$. 
gamma. unbiased Logical. If TRUE, we compute an unbiased version for the Gamma matrix. Only available for single-level complete data and when conditional. $x=$ FALSE and fixed. $x=$ FALSE (for now).

bootstrap: Number of bootstrap draws, if bootstrapping is used.

do.fit: If FALSE, the model is not fit, and the current starting values of the model parameters are preserved.

Optimization options:

control: A list containing control parameters passed to the external optimizer. By default, lavaan uses "nlminb". See the manpage of nlminb for an overview of the control parameters. If another (external) optimizer is selected, see the manpage for that optimizer to see the possible control parameters.

optim. method: Character. The optimizer that should be used. For unconstrained optimization or models with only linear equality constraints (i.e., the model syntax does not include any "==", ">" or "<" operators), the available options are "nlminb" (the default), "BFGS", "L-BFGS-B". These are all quasi-newton methods. A basic implementation of Gauss-Newton is also available (optim.method $=$ "GN"). The latter is the default when estimator = "DLS". For constrained optimization, the only available option is "nlminb.constr", which uses an augmented Lagrangian minimization algorithm.

optim. force.converged: Logical. If TRUE, pretend the model has converged, no matter what.

optim.dx.tol Numeric. Tolerance used for checking if the elements of the (unscaled) gradient are all zero (in absolute value). The default value is 0.001 .

optim.gn.tol.x: Numeric. Only used when optim. method = "GN". Optimization stops when the root mean square of the difference between the old and new parameter values are smaller than this tolerance value. Default is 1e-05 for DLS estimation and 1e-07 otherwise.

optim.gn.iter.max: Integer. Only used when optim. method = "GN". The maximum number of GN iterations. The default is 200.

bounds: Only used if optim. method = "nlminb". If logical: FALSE implies no bounds are imposed on the parameters. If TRUE, this implies bounds = "wide". If character, possible options are "none" (the default), "standard", "wide", "pos.var", "pos.ov.var", and "pos.lv.var". If bounds = "pos.ov.var", the observed variances are forced to be nonnegative. If bounds $=$ "pos.lv.var", the latent variances are forced to be nonnegative. If bounds = "pos.var", both observed and latent variances are forced to be nonnegative. If bounds = "standard", lower and upper bounds are computed for observed and latent variances, and factor loadings. If bounds = "wide", lower and upper bounds are computed for observed and latent variances, and factor loadings; but the range of the bounds is enlarged (allowing again for slightly negative variances).

optim.bounds: List. This can be used instead of the bounds argument to allow more control. Possible elements of the list are lower, upper, lower.factor and upper. factor. All of these accept a vector. The lower and upper elements indicate for which type of parameters bounds should be computed. Possible choice are "ov.var", "lv.var", "loadings" and "covariances". The lower. factor and upper. factor elements should have the same length as the lower and upper elements respectively. They indicate the factor by which the range of the bounds should be enlarged (for example, 1.1 or 1.2 ; the default is 1.0 ). Other elements are min.reliability.marker which sets the lower bound for the reliability of the 
marker indicator (if any) of each factor (default is 0.1). Finally, the min.var.lv. endo element indicates the lower bound of the variance of any endogenous latent variance (default is $0.0)$.

Parallelization options (currently only used for bootstrapping):

parallel The type of parallel operation to be used (if any). If missing, the default is "no".

ncpus Integer: number of processes to be used in parallel operation: typically one would chose this to the number of available CPUs. By By default this is the number of cores (as detected by parallel: : detectCores()) minus one.

cl An optional parallel or snow cluster for use if parallel = "snow". If not supplied, a cluster on the local machine is created for the duration of the bootstrapLavaan or bootstrapLRT call.

iseed An integer to set the seed. Or NULL if no reproducible results are needed. This works for both serial (non-parallel) and parallel settings. Internally, RNGkind() is set to "L 'Ecuyer-CMRG" if parallel = "multicore". If parallel = "snow" (under windows), parallel : : clusterSetRNGStream() is called which automatically switches to "L'Ecuyer-CMRG". When iseed is not NULL, .Random. seed (if it exists) in the global environment is left untouched.

Categorical estimation options:

zero. add: A numeric vector containing two values. These values affect the calculation of polychoric correlations when some frequencies in the bivariate table are zero. The first value only applies for $2 \times 2$ tables. The second value for larger tables. This value is added to the zero frequency in the bivariate table. If "default", the value is set depending on the "mimic" option. By default, lavaan uses zero. add $=c(0.5,0.0)$.

zero.keep.margins: Logical. This argument only affects the computation of polychoric correlations for $2 \times 2$ tables with an empty cell, and where a value is added to the empty cell. If TRUE, the other values of the frequency table are adjusted so that all margins are unaffected. If "default", the value is set depending on the "mimic". The default is TRUE.

zero.cell.warn: Logical. Only used if some observed endogenous variables are categorical. If TRUE, give a warning if one or more cells of a bivariate frequency table are empty.

Starting values options:

start: If it is a character string, the two options are currently "simple" and "Mplus". In the first case, all parameter values are set to zero, except the factor loadings and (residual) variances, which are set to one. When start is "Mplus", the factor loadings are estimated using the fabin3 estimator (tsls) per factor. The residual variances of observed variables are set tot half the observed variance, and all other (residual) variances are set to 0.05 . The remaining parameters (regression coefficients, covariances) are set to zero. If start is a fitted object of class lavaan, the estimated values of the corresponding parameters will be extracted. If it is a parameter table, for example the output of the paramaterEstimates() function, the values of the est or start or ustart column (whichever is found first) will be extracted.

Check options:

check.start: Logical. If TRUE, the starting values are checked for possibly inconsistent values (for example values implying correlations larger than one). If needed, a warning is given. 
check.gradient: Logical. If TRUE, and the model converged, a warning is given if the optimizer decided that a (local) solution has been found, while not all elements of the (unscaled) gradient (as seen by the optimizer) are (near) zero, as they should be (the tolerance used is 0.001).

check. post: Logical. If TRUE, and the model converged, a check is performed after (post) fitting, to verify if the solution is admissible. This implies that all variances are non-negative, and all the model-implied covariance matrices are positive (semi-)definite. For the latter test, we tolerate a tiny negative eigenvalue that is smaller than .Machine $\$$ double.eps ${ }^{\wedge}(3 / 4)$, treating it as being zero.

check.vcov: Logical. If TRUE, and the model converged, we check if the variance-covariance matrix of the free parameters is positive definite. We take into account possible equality and acitive inequality constraints. If needed, a warning is given.

check.lv.names: Logical. If TRUE, and latent variables are defined in the model, lavaan will stop with an error message if a latent variable name also occurs in the data (implying it is also an observed variable).

Verbosity options:

verbose: If TRUE, the function value is printed out during each iteration.

warn: If TRUE, some (possibly harmless) warnings are printed out during the iterations.

debug: If TRUE, debugging information is printed out.

Miscellaneous:

model.type: Set the model type: possible values are "cfa", "sem" or "growth". This may affect how starting values are computed, and may be used to alter the terminology used in the summary output, or the layout of path diagrams that are based on a fitted lavaan object.

mimic: If "Mplus", an attempt is made to mimic the Mplus program. If "EQS", an attempt is made to mimic the EQS program. If "default", the value is (currently) set to to "lavaan", which is very close to "Mplus".

representation: If "LISREL" the classical LISREL matrix representation is used to represent the model (using the all-y variant). No other options are available (for now).

implied: Logical. If TRUE, compute the model-implied statistics, and store them in the implied slot.

h1: Logical. If TRUE, compute the unrestricted model and store the unrestricted summary statistics (and perhaps a loglikelihood) in the h1 slot.

baseline: Logical. If TRUE, compute a baseline model (currently always the independence model, assuming all variables are uncorrelated) and store the results in the baseline slot.

baseline. conditional. $x$. free. slopes: Logical. If TRUE, and conditional. $x=$ TRUE, the (default) baseline model will allow the slopestructure to be unrestricted.

store.vcov Logical. If TRUE, and se= is not set to "none", store the full variance-covariance matrix of the model parameters in the vcov slot of the fitted lavaan object.

\section{See Also}

lavaan 


\section{Examples}

lavoptions()

lavOptions("std.lv")

lavOptions (c("std.lv", "orthogonal"))

lavPredict

Predict the values of latent variables (and their indicators).

\section{Description}

The main purpose of the lavPredict() function is to compute (or 'predict') estimated values for the latent variables in the model ('factor scores'). NOTE: the goal of this function is NOT to predict future values of dependent variables as in the regression framework! (For models with only continuous observed variables, the function lavPredictY() supports this.

\section{Usage}

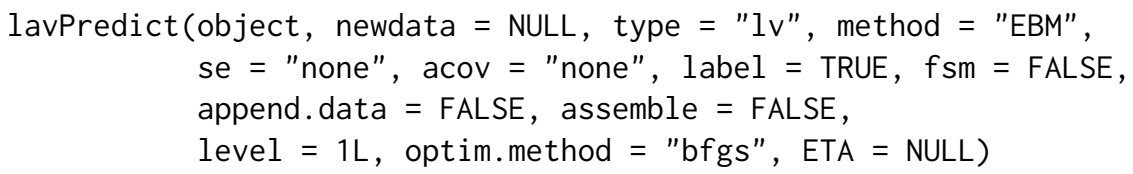

\section{Arguments}

object

newdata

type

method

se
An object of class lavaan.

An optional data.frame, containing the same variables as the data.frame used when fitting the model in object.

A character string. If " $l v "$, estimated values for the latent variables in the model are computed. If "ov", model predicted values for the indicators of the latent variables in the model are computed. If "yhat", the estimated value for the observed indicators, given user-specified values for the latent variables provided by de ETA argument. If " $f y$ ", densities (or probabilities) for each observed indicator, given user-specified values for the latent variables provided by de ETA argument.

A character string. In the linear case (when the indicators are continuous), the possible options are "regression" or "Bartlett". In the categorical case, the two options are "EBM" for the Empirical Bayes Modal approach, and "ML" for the maximum likelihood approach.

Character. If "none", no standard errors are computed. If "standard", naive standard errors are computed (assuming the parameters of the measurement model are known). The standard errors are returned as an attribute. Currently only available for complete continuous data.

Similar to the "se" argument, but optionally returns the full sampling covariance matrix of factor scores as an attribute. Currently only available for complete continuous data. 
label Logical. If TRUE, the columns in the output are labeled.

fsm

append.data

assemble

level

optim.method

ETA
Logical. If TRUE, return the factor score matrix as an attribute. Only for numeric data.

Logical. Only used when type $=" l v "$. If TRUE, the original data (or the data provided in the newdata argument) is appended to the factor scores.

Logical. If TRUE, the separate multiple groups are reassembled again to form a single data.frame with a group column, having the same dimensions are the original (or newdata) dataset.

Integer. Only used in a multilevel SEM. If level $=1$, only factor scores for latent variable defined at the first (within) level are computed; if level $=2$, only factor scores for latent variables defined at the second (between) level are computed.

Character string. Only used in the categorical case. If "nlminb" (the default in $0.5)$, the "nlminb() " function is used for the optimization. If "bfgs" or "BFGS" (the default in 0.6), the "optim()" function is used with the BFGS method.

An optional matrix or list, containing latent variable values for each observation. Used for computations when type = "ov".

\section{Details}

The predict() function calls the lavPredict() function with its default options.

If there are no latent variables in the model, type $=$ "ov" will simply return the values of the observed variables. Note that this function can not be used to 'predict' values of dependent variables, given the values of independent values (in the regression sense). In other words, the structural component is completely ignored (for now).

\section{See Also}

lavPredictY to predict $\mathrm{y}$-variables given $\mathrm{x}$-variables.

\section{Examples}

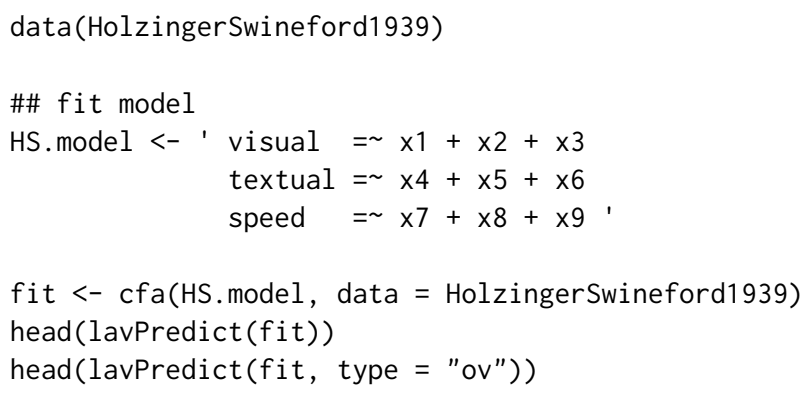




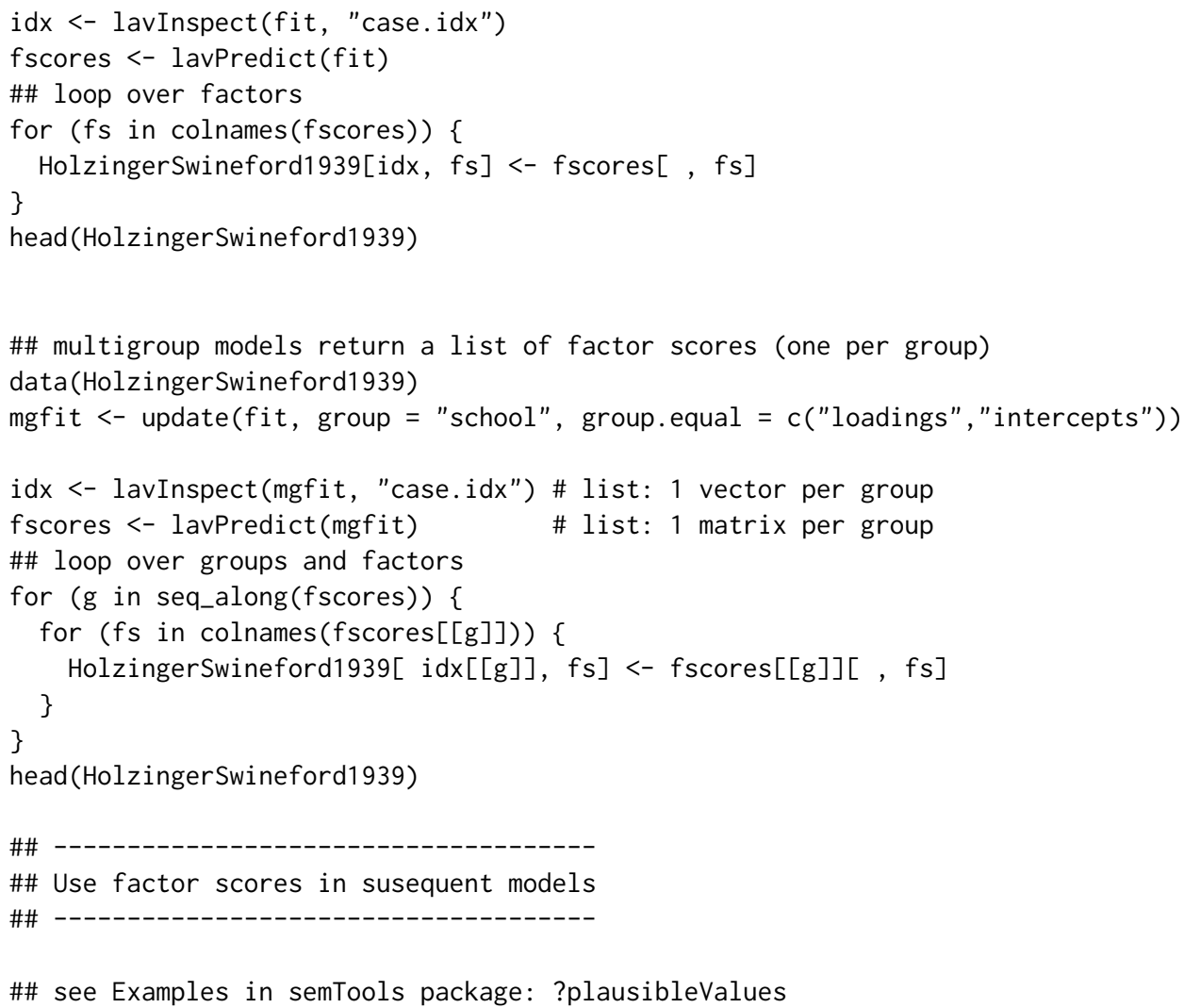

lavPredictY

Predict the values of $y$-variables given the values of $x$-variables

\section{Description}

This function can be used to predict the values of (observed) y-variables given the values of (observed) $\mathrm{x}$-variables in a structural equation model.

\section{Usage}

lavPredictY (object, newdata $=$ NULL, ynames = lavNames (object, "ov.y"), xnames $=$ lavNames (object, "ov.x"), method = "conditional.mean", label $=$ TRUE, assemble $=$ TRUE, force.zero $\cdot$ mean $=$ FALSE)

\section{Arguments}

object

An object of class lavaan. 
newdata

ynames

xnames

method

label

assemble

An optional data.frame, containing the same variables as the data.frame that was used when fitting the model in object. This data.frame should also include the $y$-variables (although their values will be ignored). Note that if no meanstructure was used in the original fit, we will use the saturated sample means of the original fit as substitutes for the model-implied means. Alternatively, refit the model using meanstructure $=$ TRUE.

The names of the observed variables that should be treated as the y-variables. It is for these variables that the function will predict the (model-based) values for each observation. Can also be a list to allow for a separate set of variable names per group (or block).

The names of the observed variables that should be treated as the $\mathrm{x}$-variables. Can also be a list to allow for a separate set of variable names per group (or block).

A character string. The only available option for now is "conditional.mean". See Details.

Logical. If TRUE, the columns of the output are labeled.

Logical. If TRUE, the predictions of the separate multiple groups in the output are reassembled again to form a single data.frame with a group column, having the same dimensions as the original (or newdata) dataset.

force.zero.mean

Logical. Only relevant if there is no mean structure. If TRUE, the (modelimplied) mean vector is set to the zero vector. If FALSE, the (model-implied) mean vector is set to the (unrestricted) sample mean vector.

\section{Details}

This function can be used for (SEM-based) out-of-sample predictions of outcome (y) variables, given the values of predictor $(\mathrm{x})$ variables. This is in contrast to the lavPredict () function which (historically) only 'predicts' the (factor) scores for latent variables, ignoring the structural part of the model.

When method = "conditional.mean", predictions (for y given $\mathrm{x}$ ) are based on the (joint $\mathrm{y}$ and $\mathrm{x})$ model-implied variance-covariance (Sigma) matrix and mean vector $(\mathrm{Mu})$, and the standard expression for the conditional mean of a multivariate normal distribution. Note that if the model is saturated (and hence $\mathrm{df}=0$ ), the SEM-based predictions are identical to ordinary least squares predictions.

\section{References}

de Rooij, M., Karch, J.D., Fokkema, M., Bakk, Z., Pratiwi, B.C, and Kelderman, H. (2022) SEMBased Out-of-Sample Predictions, Structural Equation Modeling: A Multidisciplinary Journal. DOI:10.1080/10705511.2022.2061494

\section{See Also}

lavPredict to compute scores for latent variables. 


\section{Examples}

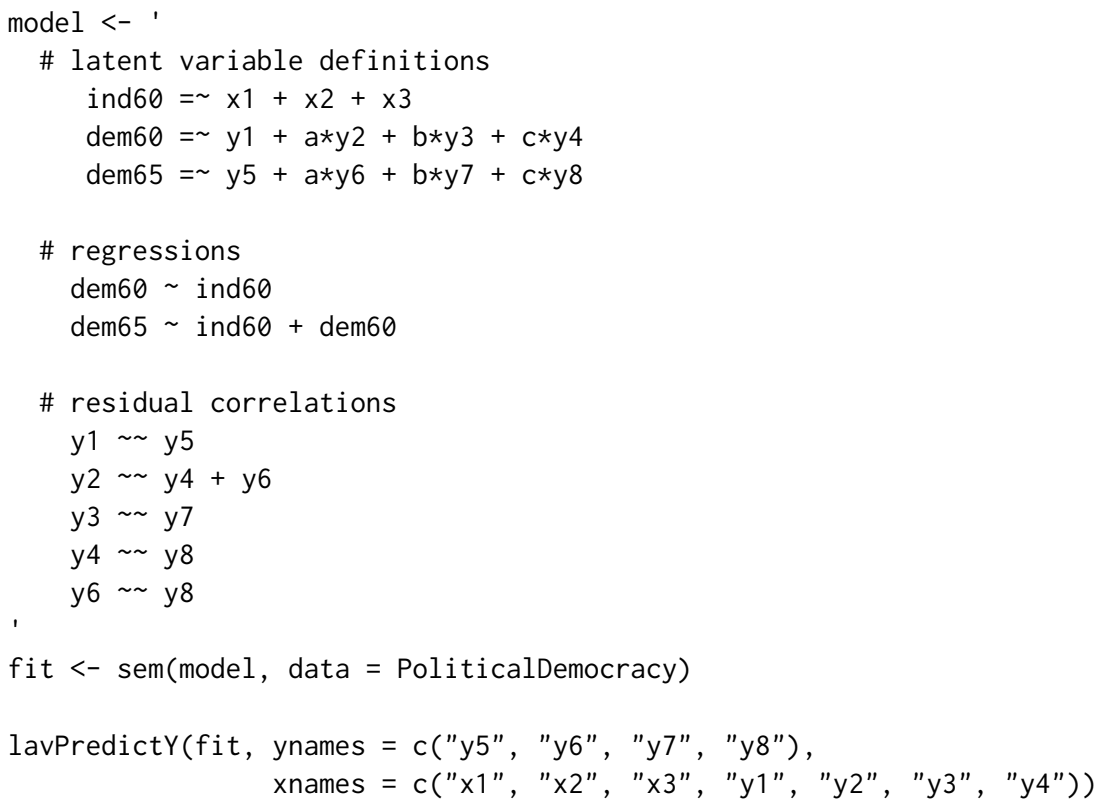

\section{Description}

'lavResiduals' provides model residuals and standardized residuals from a fitted lavaan object, as well as various summaries of these residuals.

The 'residuals()' (and 'resid()') methods are just shortcuts to this function with a limited set of arguments.

\section{Usage}

lavResiduals (object, type $=$ "cor.bentler", custom.rmr $=$ NULL, se $=$ FALSE, zstat = TRUE, summary = TRUE, h1. acov = "unstructured", add. type = TRUE, add.labels = TRUE, add. class = TRUE, drop.list. single.group = TRUE, maximum. number $=$ length $($ res. vech $)$, output $=$ "list")

\section{Arguments}

object

type
An object of class lavaan.

Character. If type $=$ "raw", this function returns the raw (= unscaled) difference between the observed and the expected (model-implied) summary statistics, as well as the standardized version of these residualds. If type $=$ "cor", or type $=$ "cor. bollen", the observed and model implied covariance matrices are first 
transformed to a correlation matrix (using $\operatorname{cov} 2 \operatorname{cor}()$ ), before the residuals are computed. If type $=$ "cor . bentler", both the observed and model implied covariance matrices are rescaled by dividing the elements by the square roots of the corresponding variances of the observed covariance matrix.

custom.rmr list. Not used yet.

se

Logical. If TRUE, show the estimated standard errors for the residuals.

zstat

Logical. If TRUE, show the standardized residuals, which are the raw residuals divided by the corresponding (estimated) standard errors.

summary

Logical. If TRUE, show various summaries of the (possibly scaled) residuals. When type = "raw", we compute the RMR. When type = "cor. bentler", we compute the SRMR. When type = "cor . bollen", we compute the CRMR. An unbiased version of these summaries is also computed, as well as a standard error, a z-statistic and a p-value for the test of exact fit based on these summaries.

h1.acov Character. If "unstructured", the observed summary statistics are used as consistent estimates of the corresponding (unrestricted) population statistics. If "structured", the model-implied summary statistics are used as consistent estimates of the corresponding (unrestricted) population statistics. This affects the way the asymptotic variance matrix of the summary statistics is computed.

add. type Logical. If TRUE, show the type of residuals in the output.

add.labels If TRUE, variable names are added to the vectors and/or matrices.

add.class If TRUE, vectors are given the 'lavaan.vector' class; matrices are given the 'lavaan.matrix' class, and symmetric matrices are given the 'lavaan.matrix.symmetric' class. This only affects the way they are printed on the screen.

drop.list.single.group

If FALSE, the results are returned as a list, where each element corresponds to a group (even if there is only a single group). If TRUE, the list will be unlisted if there is only a single group.

maximum. number Integer. Only used if output ="table". Show only the first maximum.number rows of the data.frame.

output Character. By default, output = "list", and the output is a list of elements. If output = "table", only the residuals of the variance-covariance matrix are shown in a data.frame, sorted from high (in absolute value) to low.

Value

If drop.list. single.group = TRUE, a list of (residualized) summary statistics, including type, standardized residuals, and summaries. If drop. list. single.group = FALSE, the list of summary statistics is nested within a list for each group.

\section{References}

Bentler, P.M. and Dijkstra, T. (1985). Efficient estimation via linearization in structural models. In Krishnaiah, P.R. (Ed.), Multivariate analysis - VI, (pp. 9-42). New York, NY: Elsevier.

Ogasawara, H. (2001). Standard errors of fit indices using residuals in structural equation modeling. Psychometrika, 66(3), 421-436. doi:10.1007/BF02294443 
Maydeu-Olivares, A. (2017). Assessing the size of model misfit in structural equation models. Psychometrika, 82(3), 533-558. doi:10.1007/s11336-016-9552-7

Standardized Residuals in Mplus. Document retrieved from URL http://www.statmodel.com/download/StandardizedResidua

\section{Examples}

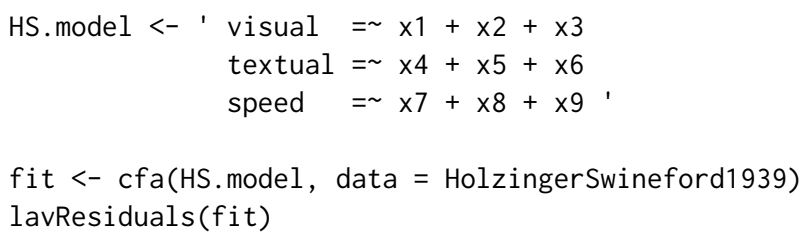

lavTables lavaan frequency tables

\section{Description}

Frequency tables for categorical variables and related statistics.

\section{Usage}

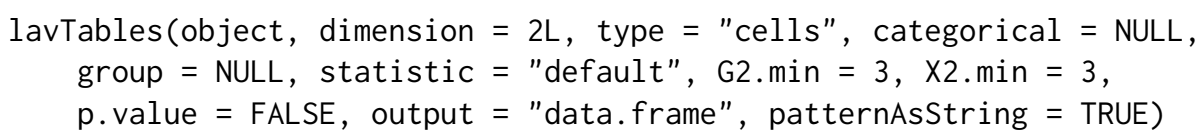

\section{Arguments}

object

dimension

type

categorical

group

statistic
Either a data. frame, or an object of class lavaan.

Integer. If $0 \mathrm{~L}$, display all response patterns. If $1 \mathrm{~L}$, display one-dimensional (one-way) tables; if 2L, display two-dimensional (two-way or pairwise) tables. For the latter, we can change the information per row: if type = "cells", each row is a cell in a pairwise table; if type = "table", each row is a table.

If "cells", display information for each cell in the (one-way or two-way) table. If "table", display information per table. If "pattern", display response patterns (implying "dimension $=0 \mathrm{~L} "$ ").

Only used if object is a data. frame. Specify variables that need to be treated as categorical.

Only used if object is a data. frame. Specify a grouping variable.

Either a character string, or a vector of character strings requesting one or more statistics for each cell, pattern or table. Always available are X2 and G2 for the Pearson and LRT based goodness-of-fit statistics. A distinction is made between the unrestricted and restricted model. The statistics based on the former have an extension *.un, as in $\mathrm{X} 2$. un and G2. un. If object is a data.frame, the unrestricted versions of the statistics are the only ones available. For oneway tables, additional statistics are the thresholds (th.un and th). For twoway tables and type $=$ "table", the following statistics are available: X2, G2, 
cor (polychoric correlation), RMSEA and the corresponding unrestricted versions (X2. un etc). Additional statistics are G2. average, G2.nlarge and G2.plarge statistics based on the cell values G2: G2 . average is the average of the G2 values in each cell of the two-way table; G2.nlarge is the number of cells with a G2 value larger than G2.min, and G2.plarge is the proportion of cells with a G2 value larger than $\mathrm{G} 2$. min. A similar set of statistics based on X2 is also available. If "default", the selection of statistics (if any) depends on the dim and type arguments, and if the object is a data. frame or a fitted lavaan object.

G2.min Numeric. All cells with a G2 statistic larger than this number are considered "large', as reflected in the (optional) "G2. plarge" and "G2.nlarge" columns.

X2.min Numeric. All cells with a X2 statistic larger than this number are considered 'large', as reflected in the (optional) "X2.plarge" and "X2.nlarge" columns.

p.value Logical. If "TRUE", p-values are computed for requested statistics (eg G2 or X2) if possible.

output If "data.frame", the output is presented as a data.frame where each row is either a cell, a table, or a response pattern, depending on the "type" argument. If "table", the output is presented as a table (or matrix) or a list of tables. Only a single statistic can be shown in this case, and if the statistic is empty, the observed frequencies are shown.

patternAsString

Logical. Only used for response patterns (dimension $=0 \mathrm{~L}$ ). If "TRUE", response patterns are displayed as a compact string. If "FALSE", as many columns as observed variables are displayed.

\section{Value}

If output = "data. frame", the output is presented as a data.frame where each row is either a cell, a table, or a response pattern, depending on the "type" argument. If output = "table" (only for two-way tables), a list of tables (if type $=$ "cells") where each list element corresponds to a pairwise table, or if type $=$ "table", a single table (per group). In both cases, the table entries are determined by the (single) statistic argument.

\section{References}

Joreskog, K.G. \& Moustaki, I. (2001). Factor analysis of ordinal variables: A comparison of three approaches. Multivariate Behavioral Research, 36, 347-387.

\section{See Also}

varTable.

\section{Examples}

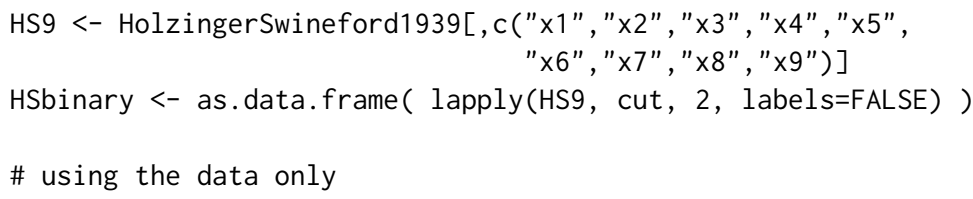




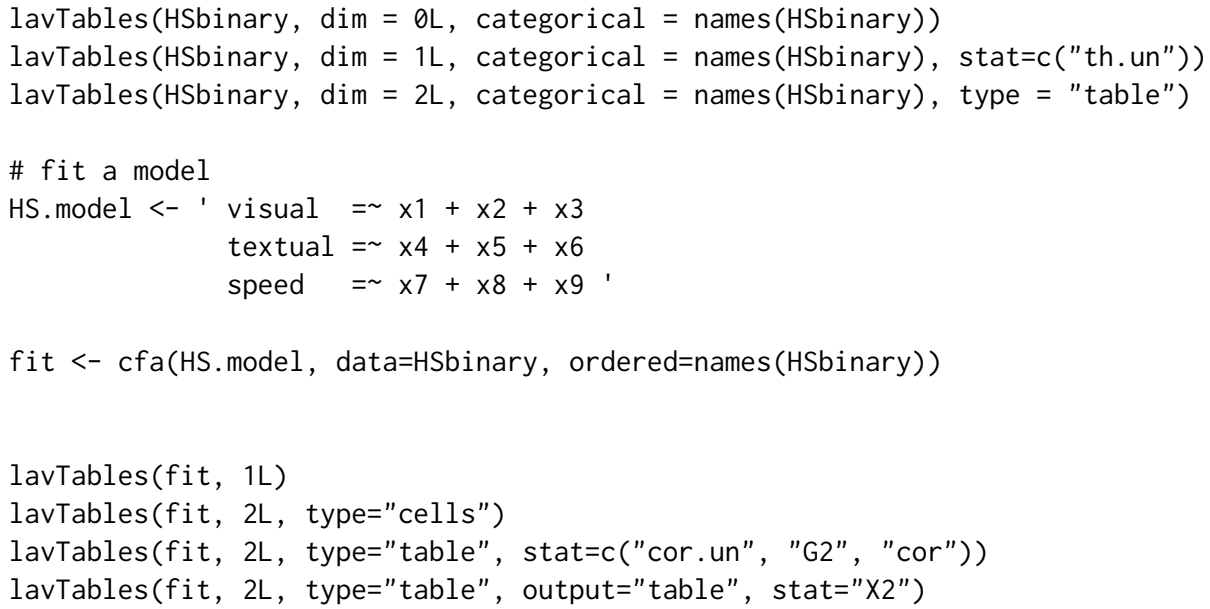

lavTablesFitcp

Pairwise maximum likelihood fit statistics

\section{Description}

Three measures of fit for the pairwise maximum likelihood estimation method that are based on likelihood ratios (LR) are defined: $C_{F}, C_{M}$, and $C_{P}$. Subscript $F$ signifies a comparison of model-implied proportions of full response patterns with observed sample proportions, subscript $M$ signifies a comparison of model-implied proportions of full response patterns with the proportions implied by the assumption of multivariate normality, and subscript $P$ signifies a comparison of model-implied proportions of pairs of item responses with the observed proportions of pairs of item responses.

\section{Usage}

lavTablesFitCf (object)

lavTablesFitCp (object, alpha $=0.05$ )

lavTablesFitCm(object)

\section{Arguments}

object An object of class lavaan.

alpha The nominal level of signifiance of global fit.

\section{Details}

$C_{F}$ : The $C_{F}$ statistic compares the log-likelihood of the model-implied proportions $\left(\pi_{r}\right)$ with the observed proportions $\left(p_{r}\right)$ of the full multivariate responses patterns:

$$
C_{F}=2 N \sum_{r} p_{r} \ln \left[p_{r} / \hat{\pi}_{r}\right]
$$


which asymptotically has a chi-square distribution with

$$
d f_{F}=m^{k}-n-1,
$$

where $k$ denotes the number of items with discrete response scales, $m$ denotes the number of response options, and $n$ denotes the number of parameters to be estimated. Notice that $C_{F}$ results may be biased because of large numbers of empty cells in the multivariate contingency table.

$C_{M}$ : The $C_{M}$ statistic is based on the $C_{F}$ statistic, and compares the proportions implied by the model of interest (Model 1) with proportions implied by the assumption of an underlying multivariate normal distribution (Model 0):

$$
C_{M}=C_{F 1}-C_{F 0}
$$

where $C_{F 0}$ is $C_{F}$ for Model 0 and $C_{F 1}$ is $C_{F}$ for Model 1. Statistic $C_{M}$ has a chi-square distribution with degrees of freedom

$$
d f_{M}=k(k-1) / 2+k(m-1)-n_{1},
$$

where $k$ denotes the number of items with discrete response scales, $m$ denotes the number of response options, and $k(k-1) / 2$ denotes the number of polychoric correlations, $k(m-1)$ denotes the number of thresholds, and $n_{1}$ is the number of parameters of the model of interest. Notice that $C_{M}$ results may be biased because of large numbers of empty cells in the multivariate contingency table. However, bias may cancels out as both Model 1 and Model 0 contain the same pattern of empty responses.

$C_{P}$ : With the $C_{P}$ statistic we only consider pairs of responses, and compare observed sample proportions $(p)$ with model-implied proportions of pairs of responses $(\pi)$. For items $i$ and $j$ we obtain a pairwise likelihood ratio test statistic $C_{P_{i j}}$

$$
C_{P_{i j}}=2 N \sum_{c_{i}=1}^{m} \sum_{c_{j}=1}^{m} p_{c_{i}, c_{j}} \ln \left[p_{c_{i}, c_{j}} / \hat{\pi}_{c_{i}, c_{j}}\right],
$$

where $m$ denotes the number of response options and $N$ denotes sample size. The $C_{P}$ statistic has an asymptotic chi-square distribution with degrees of freedom equal to the information $\left(m^{2}-1\right)$ minus the number of parameters (2(m-1) thresholds and 1 correlation),

$$
d f_{P}=m^{2}-2(m-1)-2 .
$$

As $k$ denotes the number of items, there are $k(k-1) / 2$ possible pairs of items. The $C_{P}$ statistic should therefore be applied with a Bonferroni adjusted level of significance $\alpha^{*}$, with

$$
\left.\alpha^{*}=\alpha /(k(k-1) / 2)\right),
$$

to keep the family-wise error rate at $\alpha$. The hypothesis of overall goodness-of-fit is tested at $\alpha$ and rejected as soon as $C_{P}$ is significant at $\alpha^{*}$ for at least one pair of items. Notice that with dichotomous items, $m=2$, and $d f_{P}=0$, so that hypothesis can not be tested.

\section{References}

Barendse, M. T., Ligtvoet, R., Timmerman, M. E., \& Oort, F. J. (2016). Structural Equation Modeling of Discrete data: Model Fit after Pairwise Maximum Likelihood. Frontiers in psychology, 7 , $1-8$.

Joreskog, K. G., \& Moustaki, I. (2001). Factor analysis of ordinal variables: A comparison of three approaches. Multivariate Behavioral Research, 36, 347-387. 


\section{See Also}

lavTables, lavaan

\section{Examples}

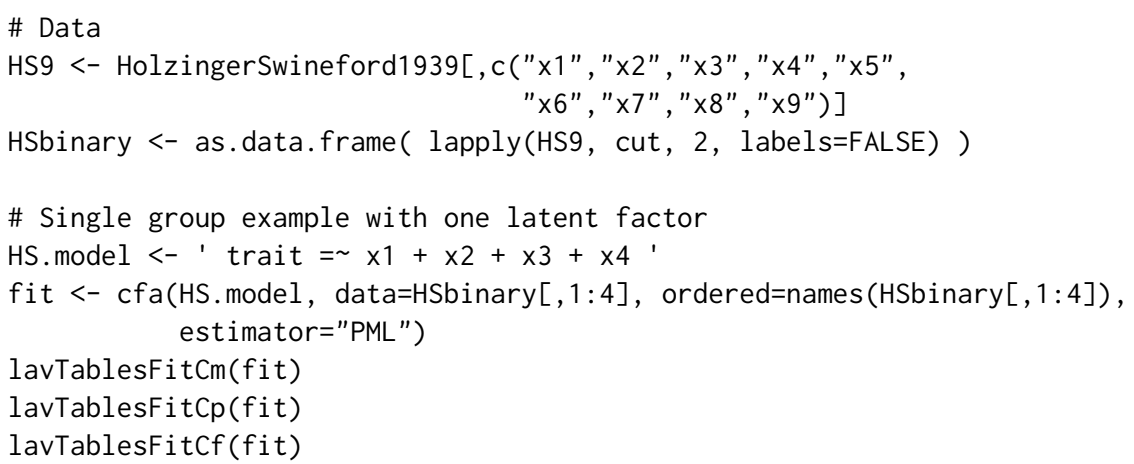

lavTest Test of exact fit

\section{Description}

Compute a variety of test statistics evaluating the global fit of the model.

Usage

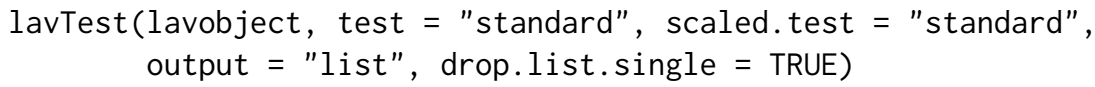

\section{Arguments}

lavobject

test
An object of class lavaan.

Character vector. Multiple names of test statistics can be provided. If "standard" is included, a conventional chi-square test is computed. If "Browne . residual . adf" is included, Browne's residual-based test statistic using ADF theory is computed. If "Browne.residual.nt" is included, Browne's residual-based test statistic using normal theory is computed. If "Satorra.Bentler" is included, a Satorra-Bentler scaled test statistic is computed. If "Yuan.Bentler" is included, a Yuan-Bentler scaled test statistic is computed. If "Yuan. Bentler. Mplus" is included, a test statistic is computed that is asymptotically equal to the YuanBentler scaled test statistic. If "mean.var.adjusted" or "Satterthwaite" is included, a mean and variance adjusted test statistic is computed. If "scaled. shifted" is included, an alternative mean and variance adjusted test statistic is computed (as in Mplus version 6 or higher). If "boot" or "bootstrap" or "Bollen. Stine" is included, the Bollen-Stine bootstrap is used to compute the bootstrap probability value of the (regular) test statistic. 
scaled.test Character. Choose the test statistic that will be scaled (if a scaled test statistic is requested). The default is "standard", but it could also be (for example) "Browne.residual.nt".

output Character. If "list" (the default), return a list with all test statistics. If "text", display the output as text with verbose descriptions (as in the summary output). If any scaled test statistics are included, they are printed first in a two-column format. Next come the other test statistics in a one-column format.

drop.list.single

Logical. Only used when output = "list". If TRUE and the list is of length one (i.e. only a single test statistic), drop the outer list. If FALSE, return a nested list with as many elements as we have test statistics.

Value

If output = "list": a nested list with test statistics, or if only a single test statistic is requested (and drop.list. single = TRUE), a list with details for this test statistic. If output = "text": the text is printed, and a nested list of test statistics (including an info attribute) is returned.

\section{Examples}

HS. model <- '

visual $=\sim \mathrm{x} 1+\mathrm{x} 2+\mathrm{x} 3$

textual $=\sim \mathrm{x} 4+\mathrm{x} 5+\mathrm{x} 6$

speed $=\sim x 7+x 8+x 9$

fit $<-c f a(H S . m o d e l$, data = HolzingerSwineford1939)

lavTest (fit, test $=$ "browne. residual.adf")

lavTestLRT LRT test

\section{Description}

LRT test for comparing (nested) lavaan models.

Usage

lavTestLRT(object, $\ldots$, method = "default", A.method = "delta", scaled. shifted $=$ TRUE, H1 = TRUE, type $=$ "Chisq", model. names $=$ NULL)

anova(object, ...)

\section{Arguments}

object

...

method
An object of class lavaan.

additional objects of class lavaan.

Character string. The possible options are "satorra. bentler . 2001", "satorra. bentler . 2010" and "satorra.2000". See details. 
H1 Not used yet

A.method Character string. The possible options are "exact" and "delta". This is only used when method = "satorra.2000". It determines how the Jacobian of the constraint function (the matrix A) will be computed. Note that if $\mathrm{A}$. method $=$ "exact", the models must be nested in the parameter sense, while if A.method $=$ "delta", they only need to be nested in the covariance matrix sense.

scaled.shifted Logical. Only used when method = "satorra.2000". If TRUE, we use a scaled and shifted test statistic; if FALSE, we use a mean and variance adjusted (Satterthwaite style) test statistic.

type Character. If "Chisq", the test statistic for each model is the (scaled or unscaled) model fit test statistic. If "Cf", the test statistic for each model is computed by the lavTablesFitcf function.

model.names Character vector. If provided, use these model names in the first column of the anova table.

\section{Details}

The anova function for lavaan objects simply calls the lavTestLRT function, which has a few additional arguments.

If type = "Chisq" and the test statistics are scaled, a special scaled difference test statistic is computed. If method is "satorra. bentler. 2001", a simple approximation is used described in Satorra $\&$ Bentler (2001). In some settings, this can lead to a negative test statistic. To ensure a positive test statistic, we can use the method proposed by Satorra \& Bentler (2010). Alternatively, when method is "satorra.2000", the original formulas of Satorra (2000) are used.

\section{Value}

An object of class anova. When given a single argument, it simply returns the test statistic of this model. When given a sequence of objects, this function tests the models against one another in the order specified.

\section{References}

Satorra, A. (2000). Scaled and adjusted restricted tests in multi-sample analysis of moment structures. In Heijmans, R.D.H., Pollock, D.S.G. \& Satorra, A. (eds.), Innovations in multivariate statistical analysis. A Festschrift for Heinz Neudecker (pp.233-247). London: Kluwer Academic Publishers.

Satorra, A., \& Bentler, P. M. (2001). A scaled difference chi-square test statistic for moment structure analysis. Psychometrika, 66(4), 507-514.

Satorra, A., \& Bentler, P. M. (2010). Ensuring postiveness of the scaled difference chi-square test statistic. Psychometrika, 75(2), 243-248.

\section{Examples}

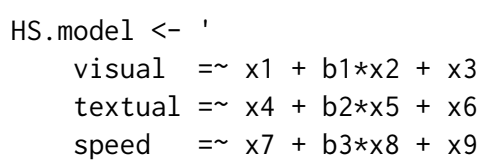


fit1 <- cfa(HS.model, data = HolzingerSwineford1939)

fito <- cfa(HS.model, data = HolzingerSwineford1939,

orthogonal $=$ TRUE)

lavTestLRT(fit1, fito)

lavTestScore Score test

\section{Description}

Score test (or Lagrange Multiplier test) for releasing one or more fixed or constrained parameters in model.

\section{Usage}

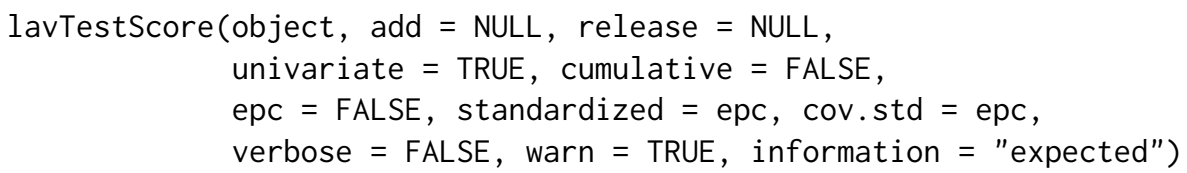

\section{Arguments}

object

add

release

univariate

cumulative

epc

standardized

cov.std
An object of class lavaan.

Either a character string (typically between single quotes) or a parameter table containing additional (currently fixed-to-zero) parameters for which the score test must be computed.

Vector of Integers. The indices of the constraints that should be released. The indices correspond to the order of the equality constraints as they appear in the parameter table.

Logical. If TRUE, compute the univariate score statistics, one for each constraints.

Logical. If TRUE, order the univariate score statistics from large to small, and compute a series of multivariate score statistics, each time adding an additional constraint.

Logical. If TRUE, and we are releasing existing constraints, compute the expected parameter changes for the existing (free) parameters, for each released constraint.

If TRUE, two extra columns (sepc.lv and sepc.all) in the \$epc table will contain standardized values for the EPCs. In the first column (sepc.lv), standardization is based on the variances of the (continuous) latent variables. In the second column (sepc.all), standardization is based on both the variances of both (continuous) observed and latent variables. (Residual) covariances are standardized using (residual) variances.

Logical. See standardizedSolution. 
verbose

warn

information
Logical. Not used for now.

Logical. If TRUE, print out warnings if they occur.

character indicating the type of information matrix to use (check lavInspect for available options). "expected" information is the default, which provides better control of Type I errors.

\section{Details}

This function can be used to compute both multivariate and univariate score tests. There are two modes: 1) releasing fixed-to-zero parameters (using the add argument), and 2) releasing existing equality constraints (using the release argument). The two modes can not be used simultaneously.

When adding new parameters, they should not already be part of the model (i.e. not listed in the parameter table). If you want to test for a parameter that was explicitly fixed to a constant (say to zero), it is better to label the parameter, and use an explicit equality constraint.

\section{Value}

A list containing at least one data. frame:

- \$test: The total score test, with columns for the score test statistic (X2), the degrees of freedom (df), and a $p$ value under the $\chi^{2}$ distribution ( $p$. value).

- \$uni: Optional (if univariate=TRUE). Each 1-df score test, equivalent to modification indices. If epc=TRUE when adding parameters (not when releasing constraints), an unstandardized EPC is provided for each added parameter, as would be returned by modificationIndices.

- \$cumulative: Optional (if cumulative=TRUE). Cumulative score tests.

- \$epc: Optional (if epc=TRUE). Parameter estimates, expected parameter changes, and expected parameter values if all the tested constraints were freed.

\section{References}

Bentler, P. M., \& Chou, C. P. (1993). Some new covariance structure model improvement statistics. Sage Focus Editions, 154, 235-255.

\section{Examples}

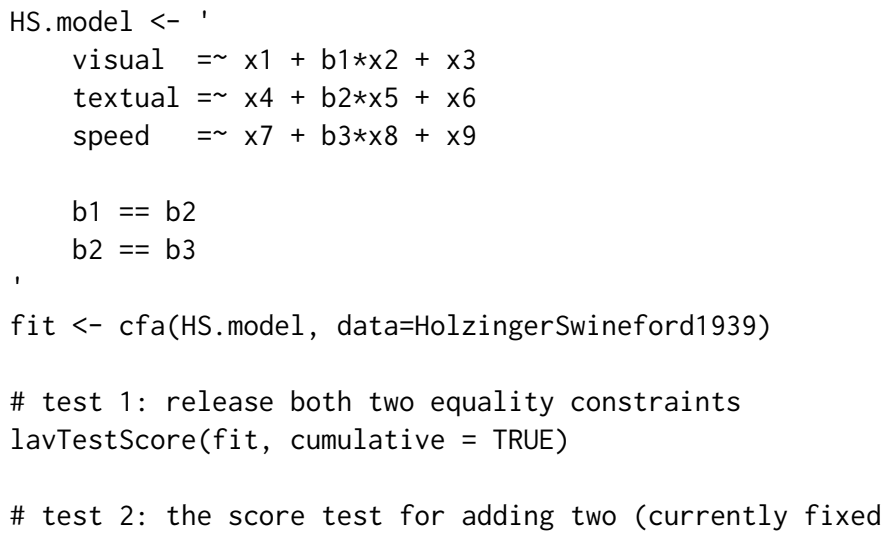




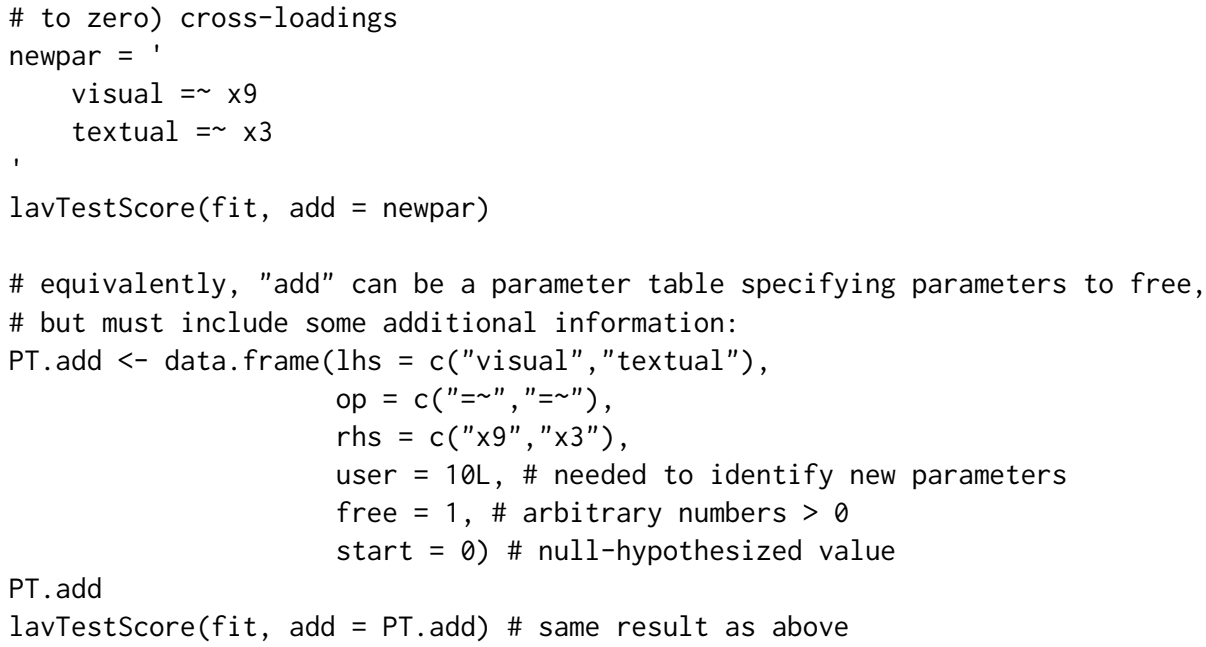

lavTestWald $\quad$ Wald test

\section{Description}

Wald test for testing a linear hypothesis about the parameters of fitted lavaan object.

\section{Usage}

lavTestWald(object, constraints $=$ NULL, verbose $=$ FALSE)

\section{Arguments}

object

constraints

verbose
An object of class lavaan.

A character string (typically between single quotes) containing one or more equality constraints. See examples for more details.

Logical. If TRUE, print out the restriction matrix and the estimated restricted values.

\section{Details}

The constraints are specified using the "==" operator. Both the left-hand side and the right-hand side of the equality can contain a linear combination of model parameters, or a constant (like zero). The model parameters must be specified by their user-specified labels. Names of defined parameters (using the ":=" operator) can be included too.

\section{Value}

A list containing three elements: the Wald test statistic (stat), the degrees of freedom (df), and a p-value under the chi-square distribution (p.value). 


\section{Examples}

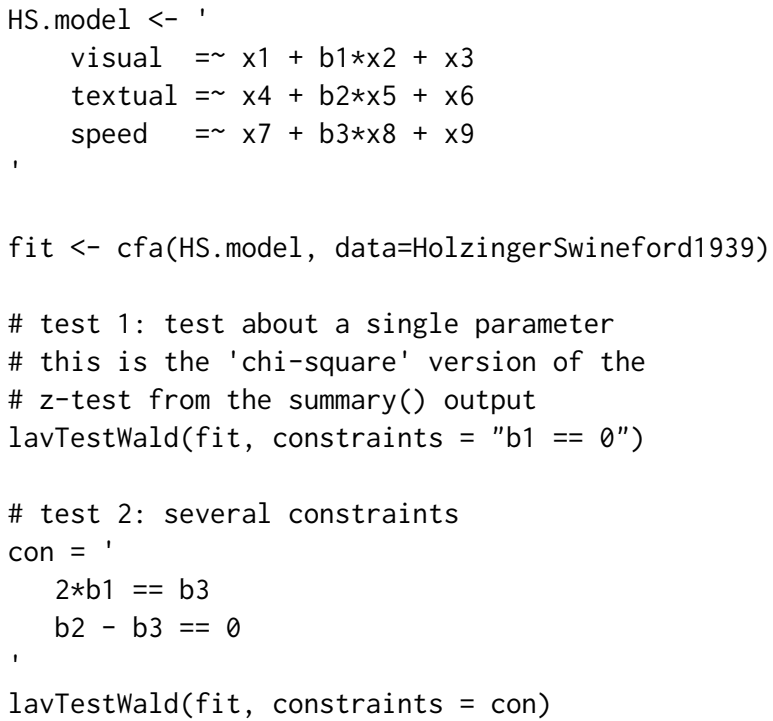

\section{Description}

Utility functions for equality and inequality constraints.

\section{Usage}

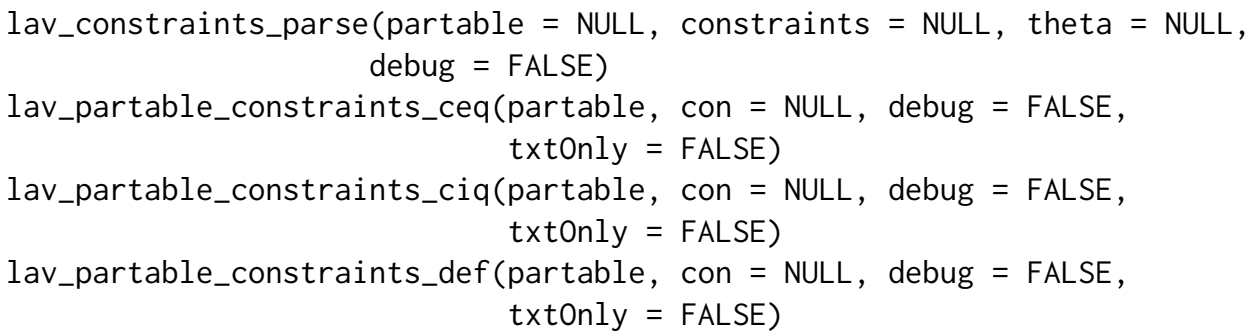

\section{Arguments}
partable
A lavaan parameter table.
constraints
A character string containing the constraints.
theta
A numeric vector. Optional vector with values for the model parameters in the parameter table.
debug
Logical. If TRUE, show debugging information.
con
An optional partable where the operator is one of '==', '> , '<' or ' $:=$ '
txtonly
Logical. If TRUE, only the body of the function is returned as a character string. If FALSE, a function is returned. 


\section{Details}

This is a collection of lower-level constraint related functions that are used in the lavaan code. They are made public per request of package developers. Below is a brief description of what they do:

The lav_constraints_parse function parses the constraints specification (provided as a string, see example), and generates a list with useful information about the constraints.

The lav_partable_constraints_ceq function creates a function which takes the (unconstrained) parameter vector as input, and returns the slack values for each equality constraint. If the equality constraints hold perfectly, this function returns zeroes.

The lav_partable_constraints_ciq function creates a function which takes the (unconstrained) parameter vector as input, and returns the slack values for each inequality constraint.

The lav_partable_constraints_def function creates a function which takes the (unconstrained) parameter vector as input, and returns the computed values of the defined parameters.

\section{Examples}

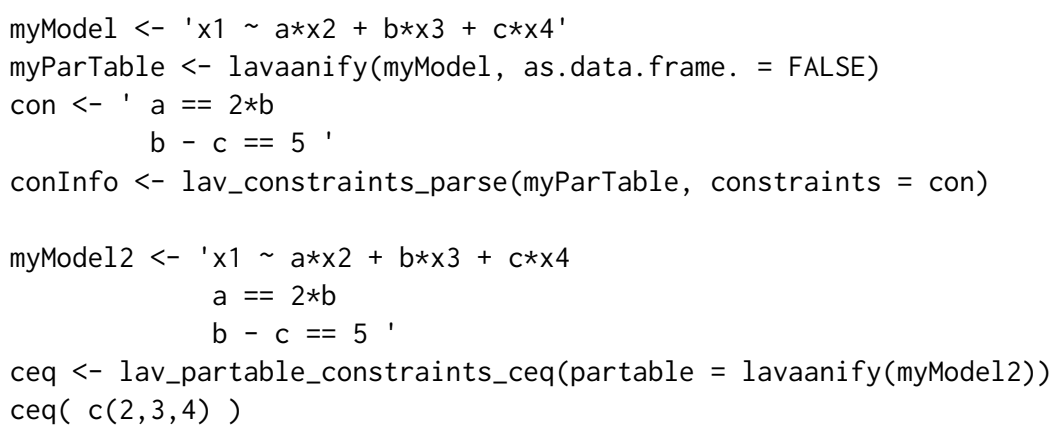

lav_data

lavaan data functions

\section{Description}

Utility functions related to the Data slot

\section{Usage}

\# update data slot with new data (of the same size)

lav_data_update $($ lavdata $=$ NULL, newX $=$ NULL, BOOT.idx $=$ NULL, lavoptions $=$ NULL $)$

\section{Arguments}

lavdata

newX

BOOT.idx

lavoptions
A lavdata object.

A list of (new) data matrices (per group) of the same size. They will replace the data stored in the internal dataslot.

A list of integers. If bootstrapping was used to produce the data in newX, use these indices to adapt the remaining slots.

A named list. The Options lsot from a lavaan object. 


\section{Examples}

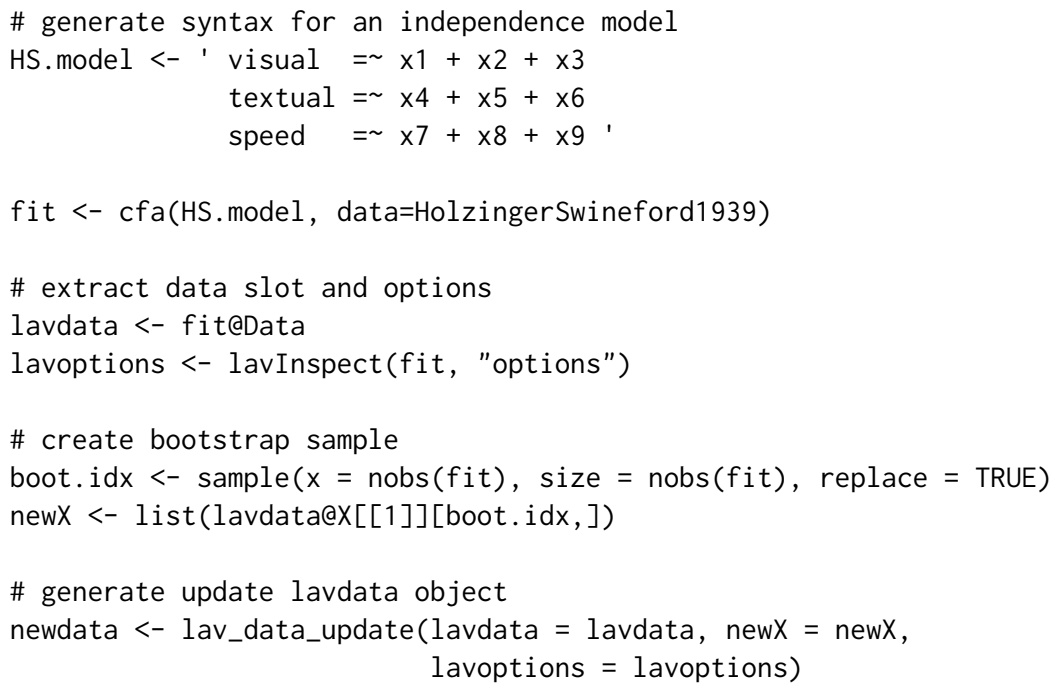

lav_func

Utility Functions: Gradient and Jacobian

\section{Description}

Utility functions for computing the gradient of a scalar-valued function or the Jacobian of a vectorvalued function by numerical approximation.

Usage

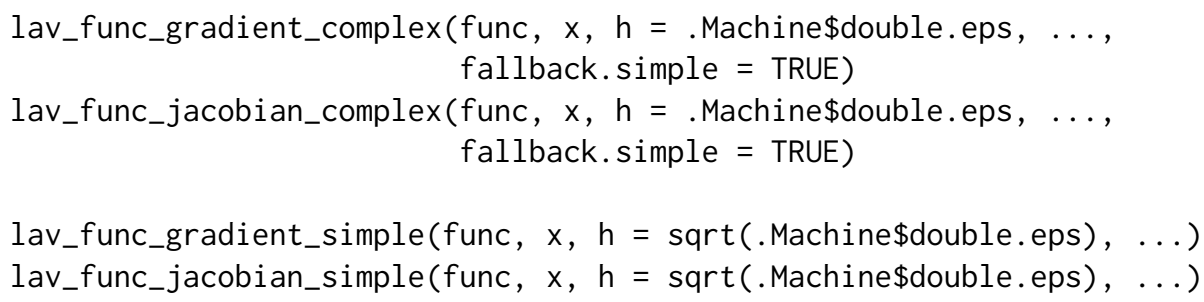

\section{Arguments}
func
$\mathrm{X}$
A real-valued function returning a numeric scalar or a numeric vector.
$x$ A numeric vector: the point(s) at which the gradient/Jacobian of the function should be computed.
$\mathrm{h} \quad$ Numeric value representing a small change in ' $\mathrm{x}$ ' when computing the gradi- ent/Jacobian.
$\cdots$
Additional arguments to be passed to the function 'func'.
fallback.simple
Logical. If TRUE, and the function evaluation fails, we call the corresponding simple (non-complex) method instead.




\section{Details}

The complex versions use complex numbers to gain more precision, while retaining the simplicity (and speed) of the simple forward method (see references). These functions were added to lavaan (around 2012) when the complex functionality was not part of the numDeriv package. They were used internally, and made public in 0.5-17 per request of other package developers.

\section{References}

Squire, W. and Trapp, G. (1998). Using Complex Variables to Estimate Derivatives of Real Functions. SIAM Review, 40(1), 110-112.

\section{Examples}

\# very accurate complex method

lav_func_gradient_complex(func $=\exp , \mathrm{x}=1)-\exp (1)$

\# less accurate forward method

lav_func_gradient_simple (func $=\exp , x=1)-\exp (1)$

\# very accurate complex method

$\operatorname{diag}\left(l_{a v}\right.$ func_jacobian_complex $($ func $\left.=\exp , \quad x=c(1,2,3))\right)-\exp (c(1,2,3))$

\# less accurate forward method

$\operatorname{diag}\left(l_{a v}\right.$ func_jacobian_simple $($ func $\left.=\exp , \quad x=c(1,2,3))\right)-\exp (c(1,2,3))$

\section{Description}

Utility functions for Matrix and Vector operations.

\section{Usage}

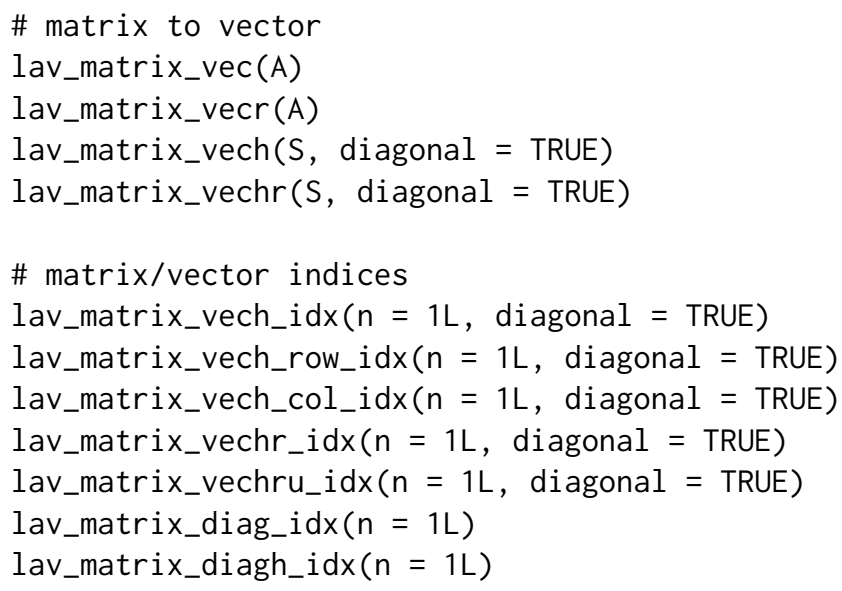




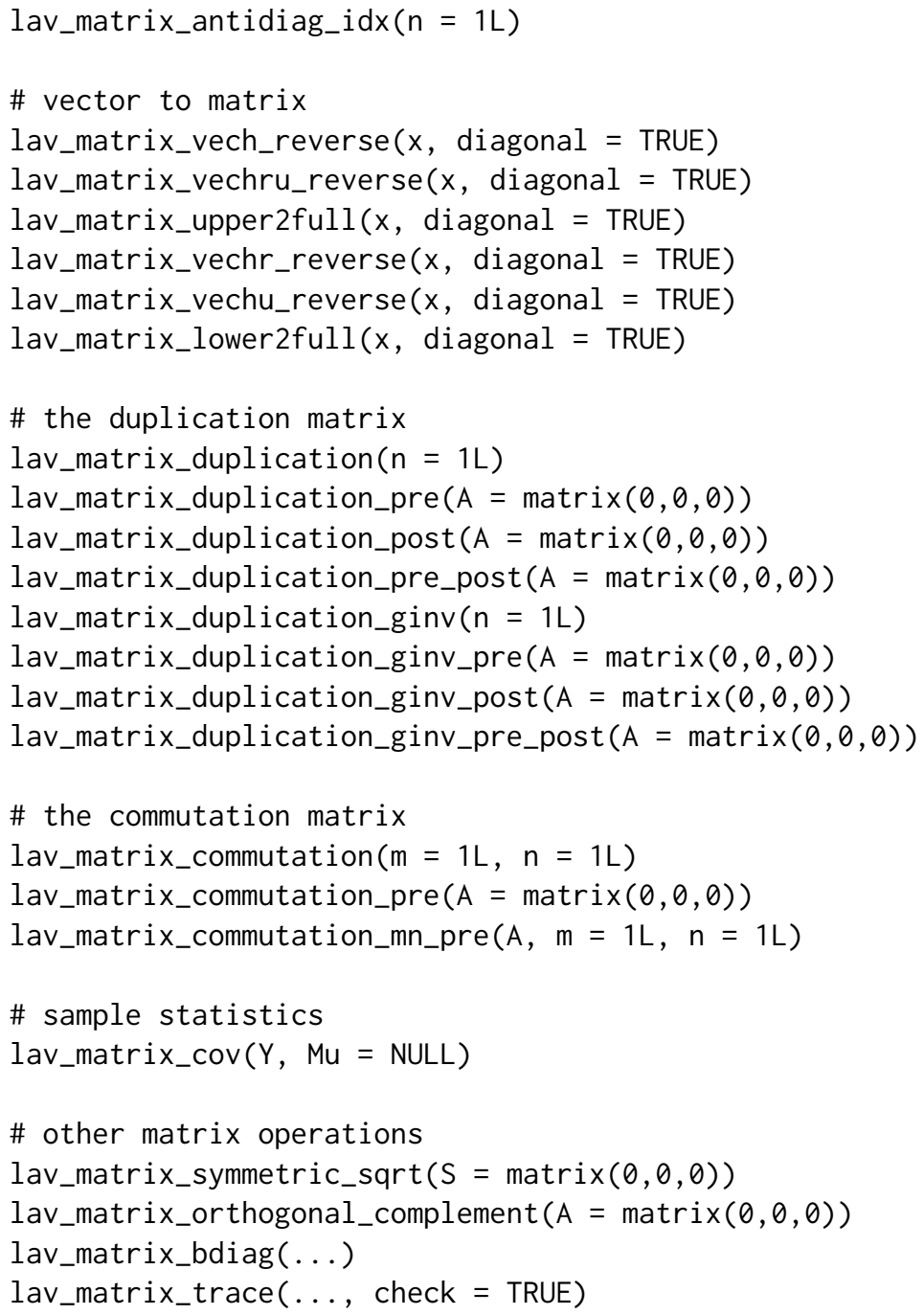

\section{Arguments}

A

S
A general matrix.

A symmetric matrix.

A matrix representing a (numeric) dataset.

Logical. If TRUE, include the diagonal.

Integer. When it is the only argument, the dimension of a square matrix. If $\mathrm{m}$ is also provided, the number of column of the matrix.

Integer. The number of rows of a matrix.

Numeric. A vector.

Numeric. If given, use $\mathrm{Mu}$ (instead of sample mean) to center, before taking the crossproduct. 
.. One or more matrices, or a list of matrices.

check Logical. If check $=$ TRUE, we check if the (final) matrix is square.

\section{Details}

These are a collection of lower-level matrix/vector related functions that are used throughout the lavaan code. They are made public per request of package developers. Below is a brief description of what they do:

The lav_matrix_vec function implements the vec operator (for 'vectorization') and transforms a matrix into a vector by stacking the columns of the matrix one underneath the other.

The lav_matrix_vecr function is similar to the lav_matrix_vec function but transforms a matrix into a vector by stacking the rows of the matrix one underneath the other.

The lav_matrix_vech function implements the vech operator (for 'half vectorization') and transforms a symmetric matrix into a vector by stacking the columns of the matrix one underneath the other, but eliminating all supradiagonal elements. If diagonal $=$ FALSE, the diagonal elements are also eliminated.

The lav_matrix_vechr function is similar to the lav_matrix_vech function but transforms a matrix into a vector by stacking the rows of the matrix one underneath the other, eliminating all supradiagonal elements.

The lav_matrix_vech_idx function returns the vector indices of the lower triangular elements of a symmetric matrix of size $\mathrm{n}$, column by column.

The lav_matrix_vech_row_idx function returns the row indices of the lower triangular elements of a symmetric matrix of size $n$.

The lav_matrix_vech_col_idx function returns the column indices of the lower triangular elements of a symmetric matrix of size $n$.

The lav_matrix_vechr_idx function returns the vector indices of the lower triangular elements of a symmetric matrix of size $\mathrm{n}$, row by row.

The lav_matrix_vechu_idx function returns the vector indices of the upper triangular elements of a symmetric matrix of size $n$, column by column.

The lav_matrix_vechru_idx function returns the vector indices of the upper triangular elements of a symmetric matrix of size $\mathrm{n}$, row by row.

The lav_matrix_diag_idx function returns the vector indices of the diagonal elements of a symmetric matrix of size $n$.

The lav_matrix_diagh_idx function returns the vector indices of the lower part of a symmetric matrix of size $n$.

The lav_matrix_antidiag_idx function returns the vector indices of the anti diagonal elements a symmetric matrix of size $\mathrm{n}$.

The lav_matrix_vech_reverse function (alias: lav_matrix_vechru_reverse and lav_matrix_upper2full) creates a symmetric matrix, given only upper triangular elements, row by row. If diagonal = FALSE, an diagonal with zero elements is added.

The lav_matrix_vechr_reverse (alias: lav_matrix_vechu_reverse and lav_matrix_lower2full) creates a symmetric matrix, given only the lower triangular elements, row by row. If diagonal $=$ FALSE, an diagonal with zero elements is added. 
The lav_matrix_duplication function generates the duplication matrix for a symmetric matrix of size $n$. This matrix duplicates the elements in $\operatorname{vech}(S)$ to create $\operatorname{vec}(S)$ (where $S$ is symmetric). This matrix is very sparse, and should probably never be explicitly created. Use one of the functions below.

The lav_matrix_duplication_pre function computes the product of the transpose of the duplication matrix and a matrix A. The A matrix should have $n^{*} n$ rows, where $n$ is an integer. The duplication matrix is not explicitly created.

The lav_matrix_duplication_post function computes the product of a matrix A with the duplication matrix. The A matrix should have $n * n$ columns, where $n$ is an integer. The duplication matrix is not explicitly created.

The lav_matrix_duplication_pre_post function first pre-multiplies a matrix A with the transpose of the duplication matrix, and then post multiplies the result again with the duplication matrix. A must be square matrix with $n * n$ rows and columns, where $n$ is an integer. The duplication matrix is not explicitly created. multiplies a matrix A with the

The lav_matrix_duplication_ginv function computes the generalized inverse of the duplication matrix. The matrix removes the duplicated elements in $\operatorname{vec}(\mathrm{S})$ to create $\operatorname{vech}(\mathrm{S})$. This matrix is very sparse, and should probably never be explicitly created. Use one of the functions below.

The lav_matrix_duplication_ginv_pre function computes the product of the generalized inverse of the duplication matrix and a matrix $A$ with $n * n$ rows, where $n$ is an integer. The generalized inverse of the duplication matrix is not explicitly created.

The lav_matrix_duplication_ginv_post function computes the product of a matrix A (with $n^{*} n$ columns, where $n$ is an integer) and the transpose of the generalized inverse of the duplication matrix. The generalized inverse of the duplication matrix is not explicitly created.

The lav_matrix_duplication_ginv_pre_post function first pre-multiplies a matrix A with the transpose of the generalized inverse of the duplication matrix, and the post multiplies the result again with the transpose of the generalized inverse matrix. The matrix A must be square with $\mathrm{n}^{*} \mathrm{n}$ rows and columns, where $\mathrm{n}$ is an integer. The generalized inverse of the duplication matrix is not explicitly created.

The lav_matrix_commutation function computes the commutation matrix which is a permutation matrix which transforms $\operatorname{vec}(\mathrm{A})$ (with $\mathrm{m}$ rows and $\mathrm{n}$ columns) into vec(t(A)).

The lav_matrix_commutation_pre function computes the product of the commutation matrix with a matrix $A$, without explicitly creating the commutation matrix. The matrix A must have $n * n$ rows, where $\mathrm{n}$ is an integer.

The lav_matrix_commutation_mn_pre function computes the product of the commutation matrix with a matrix $\mathrm{A}$, without explicitly creating the commutation matrix. The matrix A must have $\mathrm{m}^{*} \mathrm{n}$ rows, where $m$ and $n$ are integers.

The lav_matrix_cov function computes the sample covariance matrix of its input matrix, where the elements are divided by $\mathrm{N}$ (the number of rows).

The lav_matrix_symmetric_sqrt function computes the square root of a positive definite symmetric matrix (using an eigen decomposition). If some of the eigenvalues are negative, they are silently fixed to zero.

The lav_matrix_orthogonal_complement function computes an orthogonal complement of the matrix A, using a qr decomposition.

The lav_matrix_bdiag function constructs a block diagonal matrix from its arguments. 
The lav_matrix_trace function computes the trace (the sum of the diagonal elements) of a single (square) matrix, or if multiple matrices are provided (either as a list, or as multiple arguments), we first compute their product (which must result in a square matrix), and then we compute the trace; if check $=$ TRUE, we check if the (final) matrix is square.

\section{References}

Magnus, J. R. and H. Neudecker (1999). Matrix Differential Calculus with Applications in Statistics and Econometrics, Second Edition, John Wiley.

\section{Examples}

\# upper elements of a 3 by 3 symmetric matrix (row by row)

$x<-c(30,16,5,10,3,1)$

\# construct full symmetric matrix

$\mathrm{S}<-$ lav_matrix_upper2full(x)

\# compute the normal theory 'Gamma' matrix given a covariance

\# matrix (S), using the formula: Gamma $=2 * D^{\wedge}\{+\}(S \% x \% S) t\left(D^{\wedge}\{+\}\right)$

Gamma.NT <- 2 * lav_matrix_duplication_ginv_pre_post(S \% $\%$ S)

Gamma.NT

lav_model lavaan model functions

\section{Description}

Utility functions related to internal model representation (lavmodel)

\section{Usage}

\# set/get free parameters

lav_model_set_parameters(lavmodel, $x=$ NULL)

lav_model_get_parameters(lavmodel, GLIST = NULL, type = "free", extra = TRUE)

\# compute model-implied statistics

lav_model_implied(lavmodel, GLIST = NULL, delta $=$ TRUE)

\# compute standard errors

lav_model_vcov_se (lavmodel, lavpartable, VCOV $=$ NULL, BOOT $=$ NULL $)$

\section{Arguments}

lavmodel

$\mathrm{X}$

GLIST
An internal representation of a lavaan model.

Numeric.A vector containing the values of all the free model parameters.

List. A list of model matrices, similar to the output of lavInspect (object, "est"). 
type Character string. If "free", only return the free model parameters. If "user", return all the parameters (free and fixed) as they appear in the user-specified parameter table.

extra Logical. If TRUE, also include values for rows in the parameter table where the operator is one of ":=", "==", "<" or ">".

delta Logical. If TRUE, and a Delta matrix is present in GLIST, use the (diagonal) values of the Delta matrix to rescale the covariance matrix. This is usually needed in the categorical setting to convert covariances to correlations.

lavpartable A parameter table.

VCOV Numeric matrix containing an estimate of the variance covariance matrix of the free model parameters.

BOOT Numeric matrix containing the bootstrap based parameter estimates (in the columns) for each bootstrap sample (in the rows).

\section{Examples}

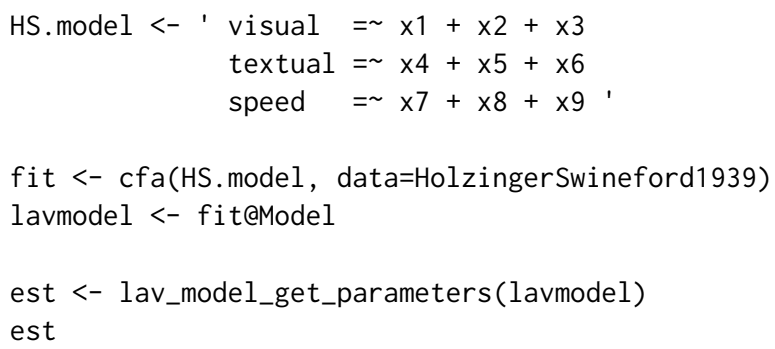

\section{Description}

Utility functions related to the parameter table (partable)

\section{Usage}

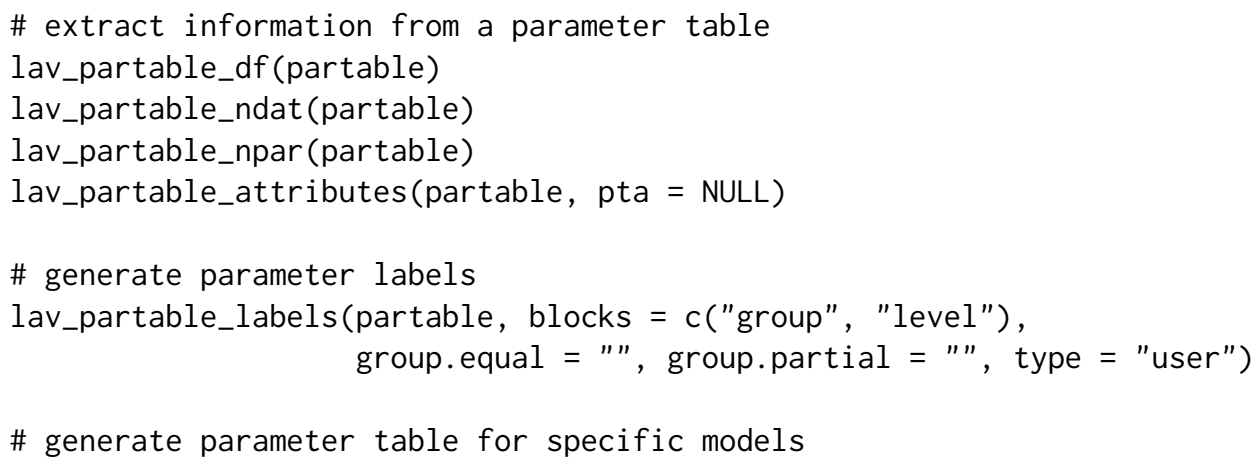




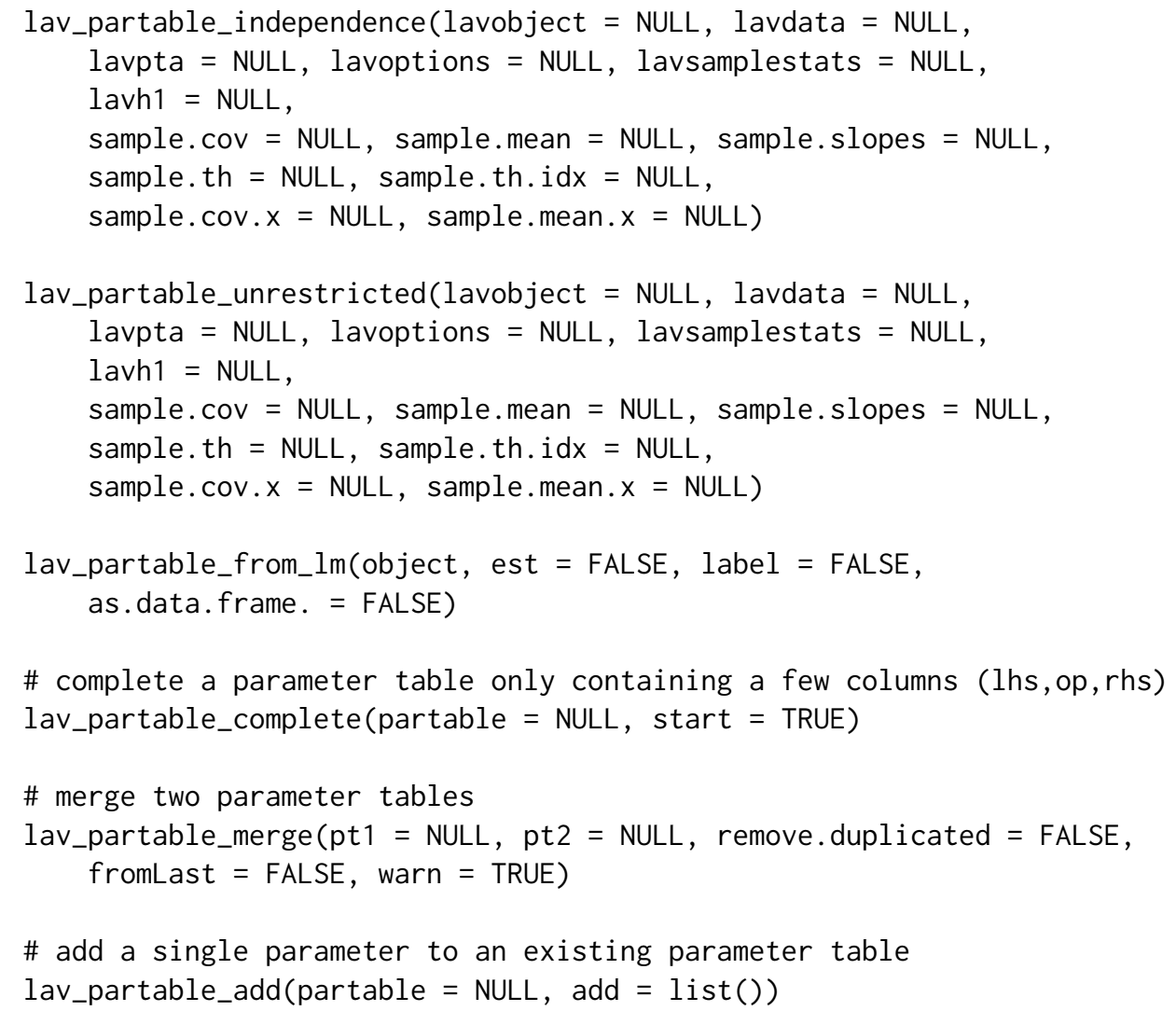

\section{Arguments}

partable

blocks

group.equal

group.partial

type

lavobject

lavdata

lavoptions
A parameter table. see lavParTable for more information.

Character vector. Which columns in the parameter table should be taken to distinguish between different blocks of parameters (and hence be given different labels)? If "blocks" includes "group", a suffix ".g" and the group number (or group label) is added for the parameters of all but the first group. If "blocks" includes "level", a suffix ". l" and the level number is added for the parameters of all but the first level. If "blocks" includes, say "foo", a suffix ". foo" and the corresponding value of "foo" is added to all parameters.

The same options can be used here as in the fitting functions. Parameters that are constrained to be equal across groups will be given the same label.

A vector of character strings containing the labels of the parameters which should be free in all groups.

Character string. Can be either 'user' or 'free' to select all entries or only the free parameters from the parameter table respectively.

An object of class 'lavaan'. If this argument is provided, it should be the only argument. All the values for the other arguments are extracted from this object.

An object of class 'lavData'. The Data slot from a lavaan object.

A named list. The Options lsot from a lavaan object. 
lavsamplestats An object of class 'lavSampleStats'. The SampleStats slot from a lavaan object.

lavh1 A named list. The h1 slot from a lavaan object.

lavpta The pta (parameter table attributes) slot from a lavaan object.

sample.cov Optional list of numeric matrices. Each list element contains a sample variancecovariance matrix for this group. If provided, these values will be used as starting values.

sample.mean Optional list of numeric vectors. Each list element contains a sample mean vector for this group. If provided, these values will be used as starting values.

sample.slopes Optional list of numeric matrices. Each list element contains the sample slopes for this group (only used when conditional. $x=$ TRUE). If provided, these values will be used as starting values.

sample.th Optional list of numeric vectors. Each list element contains a vector of sample thresholds for this group. If provided (and also sample.th.idx is provided), these values will be used as starting values.

sample. th.idx Optional list of integers. Each list contains the threshold indices for this group.

sample.cov.x Optional list of numeric matrices. Each list element contains a sample variancecovariance matrix for the exogenous variables for this group (only used when conditional. $x=$ TRUE). If provided, these values will be used as starting values.

sample.mean. $x$ Optional list of numeric vectors. Each list element contains a sample mean vector for the exogenous variables for this group (only used when conditional. $x$ $=$ TRUE). If provided, these values will be used as starting values.

est Logical. If TRUE, include the fitted estimates in the parameter table.

label Logical. If TRUE, include parameter labels in the parameter table.

as . data. frame. Logical. If TRUE, return the parameter table as a data.frame.

object An object of class $1 \mathrm{~m}$.

start Logical. If TRUE, include a start column, based on the simple method for generating starting values.

pta A list containing parameter attributes.

pt1 A parameter table.

pt2 A parameter table.

remove.duplicated

Logical. If TRUE, remove duplicated elements when merging two parameter tables.

fromLast Logical. If TRUE, duplicated elements are considered from the bottom of the merged parameter table.

warn Logical. If codeTRUE, a warning is produced when duplicated elements are found, when merging two parameter tables.

add A named list.A single row of a parameter table as a named list. 


\section{Examples}

\# generate syntax for an independence model

HS. model $<-1$ visual $=\sim x 1+x 2+x 3$

textual $=\sim x 4+x 5+x 6$

speed $=\sim x 7+x 8+x 9 '$

fit $<-$ cfa(HS.model, data=HolzingerSwineford1939)

lav $<-$ lav_partable_independence(fit)

as. data. frame (lav, stringsAsFactors $=$ FALSE)

\# how many free parameters?

lav_partable_npar(lav)

\# how many sample statistics?

lav_partable_ndat(lav)

lav_samplestats lavaan samplestats functions

\section{Description}

Utility functions related to the sample statistics

Usage

\# generate samplestats object from full data

lav_samplestats_from_data(lavdata $=$ NULL, lavoptions $=$ NULL,

WLS.V = NULL, NACOV $=$ NULL)

\section{Arguments}

lavdata

lavoptions

WLS.V

NACOV
A lavdata object.

A named list. The Options lsot from a lavaan object.

A user provided weight matrix.

A user provided matrix containing the elements of ( $\mathrm{N}$ times) the asymptotic variance-covariance matrix of the sample statistics. For a multiple group analysis, a list with an asymptotic variance-covariance matrix for each group.

\section{Examples}

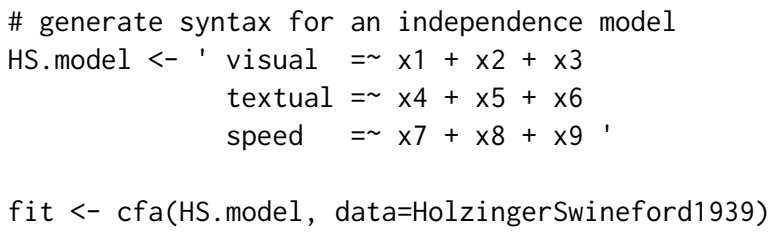


\# extract data slot and options

lavdata <- fit@Data

lavoptions <- lavInspect(fit, "options")

\# generate sample statistics object

sampleStats <- lav_samplestats_from_data(lavdata = lavdata, lavoptions = lavoptions)

model.syntax

The Lavaan Model Syntax

\section{Description}

The lavaan model syntax describes a latent variable model. The function lavaanify turns it into a table that represents the full model as specified by the user. We refer to this table as the parameter table.

\section{Usage}

lavaanify (model $=$ NULL, meanstructure $=$ FALSE, int.ov.free $=$ FALSE, int.lv.free $=$ FALSE, orthogonal $=$ FALSE, orthogonal. $y=F A L S E$, orthogonal. $x=$ FALSE, orthogonal. efa $=$ FALSE, std.lv $=$ FALSE, correlation $=$ FALSE, effect. coding $="$ ", conditional. $x=$ FALSE, fixed. $x=$ FALSE, parameterization = "delta", constraints $=$ NULL, ceq. simple $=$ FALSE, auto = FALSE, model.type $=$ "sem", auto.fix.first = FALSE, auto.fix.single = FALSE, auto.var $=$ FALSE, auto.cov.lv.x = FALSE, auto.cov. $y=$ FALSE, auto.th $=$ FALSE, auto.delta $=$ FALSE, auto. efa $=$ FALSE, varTable $=$ NULL, ngroups $=1 \mathrm{~L}$, nthresholds $=$ NULL, group. equal $=$ NULL, group.partial $=$ NULL, group.w.free $=$ FALSE, debug = FALSE, warn = TRUE, as.data.frame. = TRUE)

lavParTable (model $=$ NULL, meanstructure $=$ FALSE, int.ov.free $=$ FALSE, int.lv.free $=$ FALSE, orthogonal $=$ FALSE, orthogonal. $y=F A L S E$, orthogonal. $x=$ FALSE, orthogonal. efa $=$ FALSE, std.lv $=$ FALSE, correlation = FALSE, effect. coding $="$ ", conditional. $x=$ FALSE, fixed. $x$ = FALSE, parameterization = "delta", constraints $=$ NULL, ceq. simple $=$ FALSE, auto = FALSE, model.type $=$ "sem", auto.fix.first = FALSE, auto.fix.single = FALSE, auto.var $=$ FALSE, auto.cov.lv. $x=$ FALSE, auto. $\operatorname{cov} \cdot y=$ FALSE, auto.th $=$ FALSE, auto. delta $=$ FALSE, auto. efa $=$ FALSE, varTable $=$ NULL, ngroups $=1 \mathrm{~L}$, nthresholds $=$ NULL, group. equal $=$ NULL, group.partial $=$ NULL, group.w.free $=$ FALSE, debug = FALSE, warn = TRUE, as.data.frame. = TRUE)

lavParseModelString (model.syntax $=$ ' ', as.data.frame. = FALSE, warn = TRUE, debug $=$ FALSE) 


\section{Arguments}

model

A description of the user-specified model. Typically, the model is described using the lavaan model syntax; see details for more information. Alternatively, a parameter table (e.g., the output of lavParseModelString is also accepted.

model.syntax The model syntax specifying the model. Must be a literal string.

meanstructure If TRUE, intercepts/means will be added to the model both for both observed and latent variables.

int.ov. free If FALSE, the intercepts of the observed variables are fixed to zero.

int.lv. free If FALSE, the intercepts of the latent variables are fixed to zero.

or thogonal If TRUE, all covariances among latent variables are set to zero.

or thogonal.y If TRUE, all covariances among endogenous latent variables only are set to zero.

or thogonal.x If TRUE, all covariances among exogenous latent variables only are set to zero.

or thogonal.efa If TRUE, all covariances among latent variables involved in rotation only are set to zero.

std.lv If TRUE, the metric of each latent variable is determined by fixing their variances to 1.0. If FALSE, the metric of each latent variable is determined by fixing the factor loading of the first indicator to 1.0. If there are multiple groups, std. $1 \mathrm{v}=$ TRUE and "loadings" is included in the group. label argument, then only the latent variances i of the first group will be fixed to 1.0, while the latent variances of other groups are set free.

correlation If TRUE, a correlation structure is fitted. For continuous data, this implies that the (residual) variances are no longer parameters of the model.

effect.coding Can be logical or character string. If logical and TRUE, this implies effect. coding = c ("loadings", "intercepts"). If logical and FALSE, it is set equal to the empty string. If "loadings" is included, equality constraints are used so that the average of the factor loadings (per latent variable) equals 1 . Note that this should not be used together with std. lv = TRUE. If "intercepts" is included, equality constraints are used so that the sum of the intercepts (belonging to the indicators of a single latent variable) equals zero. As a result, the latent mean will be freely estimated and usually equal the average of the means of the involved indicators.

conditional. $x$ If TRUE, we set up the model conditional on the exogenous ' $x$ ' covariates; the model-implied sample statistics only include the non-X variables. If FALSE, the exogenous ' $x$ ' variables are modeled jointly with the other variables, and the model-implied statistics refect both sets of variables.

fixed. $x$

If TRUE, the exogenous ' $x$ ' covariates are considered fixed variables and the means, variances and covariances of these variables are fixed to their sample values. If FALSE, they are considered random, and the means, variances and covariances are free parameters.

parameterization

Currently only used if data is categorical. If "delta", the delta parameterization is used. If "theta", the theta parameterization is used.

constraints Additional (in)equality constraints. See details for more information. 
ceq.simple

auto

model. type

auto.fix.first

auto.fix.single

If TRUE, the residual variance (if included) of an observed indicator is set to zero if it is the only indicator of a latent variable.

auto.var If TRUE, the residual variances and the variances of exogenous latent variables are included in the model and set free.

auto.cov.lv.x If TRUE, the covariances of exogenous latent variables are included in the model and set free.

auto.cov.y If TRUE, the covariances of dependent variables (both observed and latent) are included in the model and set free.

auto.th If TRUE, thresholds for limited dependent variables are included in the model and set free.

auto.delta

auto.efa

varTable

ngroups

nthresholds

group. equal

group.partial
If TRUE, response scaling parameters for limited dependent variables are included in the model and set free.

If TRUE, the necessary constraints are imposed to make the (unrotated) exploratory factor analysis blocks identifiable: for each block, factor variances are set to 1 , factor covariances are constrained to be zero, and factor loadings are constrained to follow an echelon pattern.

The variable table containing information about the observed variables in the model.

The number of (independent) groups.

Either a single integer or a named vector of integers. If nthresholds is a single integer, all endogenous variables are assumed to be ordered with nthresholds indicating the number of thresholds needed in the model. If nthresholds is a named vector, it indicates the number of thresholds for these ordered variables only. This argument should not be used in combination with varTable.

A vector of character strings. Only used in a multiple group analysis. Can be one or more of the following: "loadings", "intercepts", "means", "regressions", "residuals" or "covariances", specifying the pattern of equality constraints across multiple groups. When (in the model syntax) a vector of labels is used as a modifier for a certain parameter, this will override the group.equal setting if it applies to this parameter. See also the Multiple groups section below for using modifiers in multiple groups.

A vector of character strings containing the labels of the parameters which should be free in all groups (thereby overriding the group.equal argument for some specific parameters). 
group.w. free Logical. If TRUE, the group frequencies are considered to be free parameters in the model. In this case, a Poisson model is fitted to estimate the group frequencies. If FALSE (the default), the group frequencies are fixed to their observed values.

warn If TRUE, some (possibly harmless) warnings are printed out.

as. data. frame. If TRUE, return the list of model parameters as a data. frame.

debug If TRUE, debugging information is printed out.

\section{Details}

The model syntax consists of one or more formula-like expressions, each one describing a specific part of the model. The model syntax can be read from a file (using readLines), or can be specified as a literal string enclosed by single quotes as in the example below.

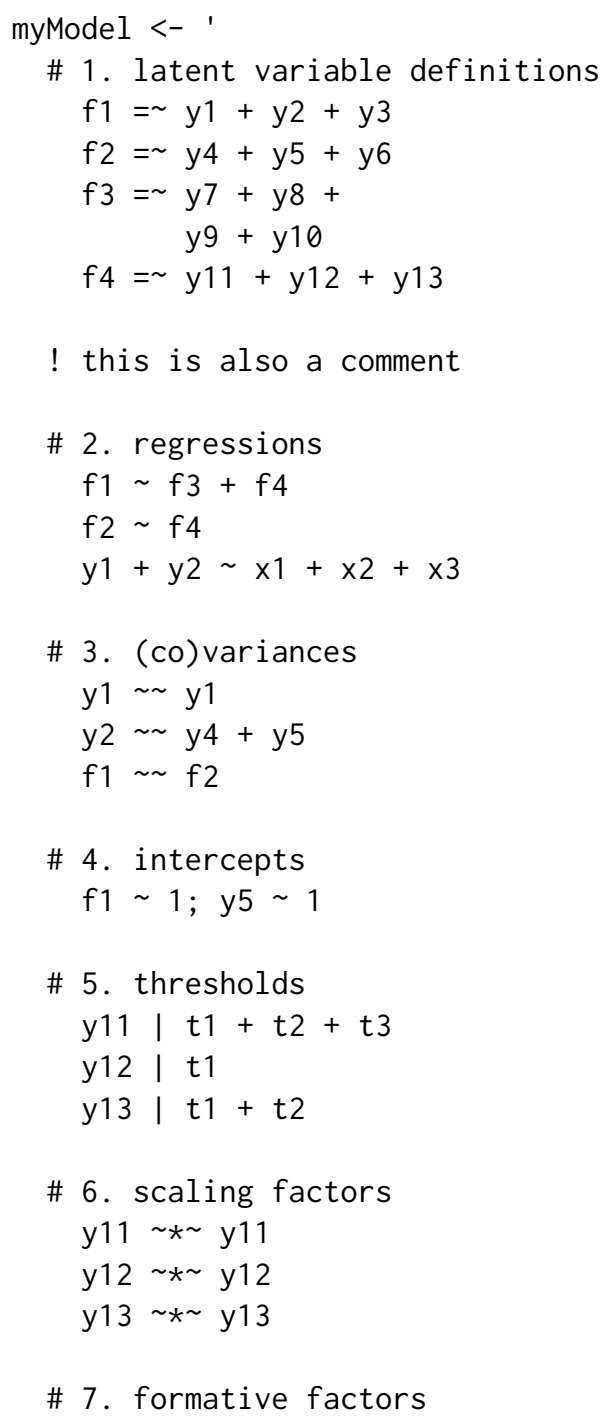

\# 7. formative factors 
$f 5<\sim z 1+z 2+z 3+z 4$

Blank lines and comments can be used in between the formulas, and formulas can be split over multiple lines. Both the sharp (\#) and the exclamation (!) characters can be used to start a comment. Multiple formulas can be placed on a single line if they are separated by a semicolon (;).

There can be seven types of formula-like expressions in the model syntax:

1. Latent variable definitions: The "= " operator can be used to define (continuous) latent variables. The name of the latent variable is on the left of the "= " operator, while the terms on the right, separated by "+" operators, are the indicators of the latent variable.

The operator " = " can be read as "is manifested by".

2. Regressions: The " " operator specifies a regression. The dependent variable is on the left of a " " operator and the independent variables, separated by "+" operators, are on the right. These regression formulas are similar to the way ordinary linear regression formulas are used in R, but they may include latent variables. Interaction terms are currently not supported.

3. Variance-covariances: The " " ('double tilde') operator specifies (residual) variances of an observed or latent variable, or a set of covariances between one variable, and several other variables (either observed or latent). Several variables, separated by "+" operators can appear on the right. This way, several pairwise (co)variances involving the same left-hand variable can be expressed in a single expression. The distinction between variances and residual variances is made automatically.

4. Intercepts: A special case of a regression formula can be used to specify an intercept (or a mean) of either an observed or a latent variable. The variable name is on the left of a " " operator. On the right is only the number "1" representing the intercept. Including an intercept formula in the model automatically implies meanstructure = TRUE. The distinction between intercepts and means is made automatically.

5. Thresholds: The "I" operator can be used to define the thresholds of categorical endogenous variables (on the left hand side of the operator). By convention, the thresholds (on the right hand sided, separated by the "+" operator, are named " $t 1$ ", " $t 2$ ", etcetera.

6. Scaling factors: The " * " operator defines a scale factor. The variable name on the left hand side must be the same as the variable name on the right hand side. Scale factors are used in the Delta parameterization, in a multiple group analysis when factor indicators are categorical.

7. Formative factors: The "< " operator can be used to define a formative factor (on the right hand side of the operator), in a similar way to how a reflexive factor is defined (using the "= " operator). This is just syntax sugar to define a phantom latent variable (equivalent to using " $f$ $=\sim \theta^{\prime \prime}$. And in addition, the (residual) variance of the formative factor is fixed to zero.

Usually, only a single variable name appears on the left side of an operator. However, if multiple variable names are specified, separated by the " + " operator, the formula is repeated for each element on the left side (as for example in the third regression formula in the example above). The only exception are scaling factors, where only a single element is allowed on the left hand side.

In the right-hand side of these formula-like expressions, each element can be modified (using the " $\star$ " operator) by either a numeric constant, an expression resulting in a numeric constant, an expression resulting in a character vector, or one of three special functions: start(), label() and equal(). This provides the user with a mechanism to fix parameters, to provide alternative starting values, to label the parameters, and to define equality constraints among model parameters. All "*" expressions are referred to as modifiers. They are explained in more detail in the following sections. 
model.syntax

\section{Fixing parameters}

It is often desirable to fix a model parameter that is otherwise (by default) free. Any parameter in a model can be fixed by using a modifier resulting in a numerical constaint. Here are some examples:

- Fixing the regression coefficient of the predictor $\times 2$ :

$y \sim x 1+2.4 * x 2+x 3$

- Specifying an orthogonal (zero) covariance between two latent variables:

$f 1 \sim 0 * f 2$

- Specifying an intercept and a linear slope in a growth model:

$\mathrm{i}=\sim 1 * \mathrm{y} 11+1 * \mathrm{y} 12+1 * \mathrm{y} 13+1 * \mathrm{y} 14$

$s=\sim 0 * y 11+1 * y 12+2 * y 13+3 * y 14$

Instead of a numeric constant, one can use a mathematical function that returns a numeric constant, for example sqrt (10). Multiplying with NA will force the corresponding parameter to be free.

\section{Starting values}

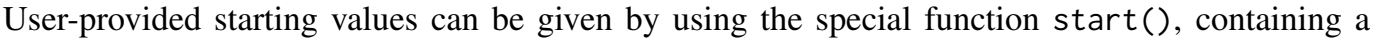
numeric constant. For example:

$y \sim x 1+\operatorname{start}(1.0) * x 2+x 3$

Note that if a starting value is provided, the parameter is not automatically considered to be free.

\section{Parameter labels and equality constraints}

Each free parameter in a model is automatically given a name (or label). The name given to a model parameter consists of three parts, coerced to a single character vector. The first part is the name of the variable in the left-hand side of the formula where the parameter was implied. The middle part is based on the special 'operator' used in the formula. This can be either one of "= ", " " or " ". The third part is the name of the variable in the right-hand side of the formula where the parameter was implied, or "1" if it is an intercept. The three parts are pasted together in a single string. For example, the name of the fixed regression coefficient in the regression formula $y \sim x 1+2.4 * x 2+$ $x 3$ is the string " $y \sim 22$ ". The name of the parameter corresponding to the covariance between two latent variables in the formula $f 1 \sim \sim \mathrm{f} 2$ is the string " $\mathrm{f} 1 \sim \sim \mathrm{f} 2$ ".

Although this automatic labeling of parameters is convenient, the user may specify its own labels for specific parameters simply by pre-multiplying the corresponding term (on the right hand side of the operator only) by a character string (starting with a letter). For example, in the formula $f 1=\sim x 1+x 2+$ mylabel $1 * x 3$, the parameter corresponding with the factor loading of $x 3$ will be named "mylabel". An alternative way to specify the label is as follows: $f 1=\sim x 1+x 2+$ label ("mylabel") *x3, where the label is the argument of special function label(); this can be useful if the label contains a space, or an operator (like " $"$ ").

To constrain a parameter to be equal to another target parameter, there are two ways. If you have specified your own labels, you can use the fact that equal labels imply equal parameter values. If you rely on automatic parameter labels, you can use the special function equal(). The argument of equal() is the (automatic or user-specified) name of the target parameter. For example, in the 
confirmatory factor analysis example below, the intercepts of the three indicators of each latent variable are constrained to be equal to each other. For the first three, we have used the default names. For the last three, we have provided a custom label for the y2a intercept.

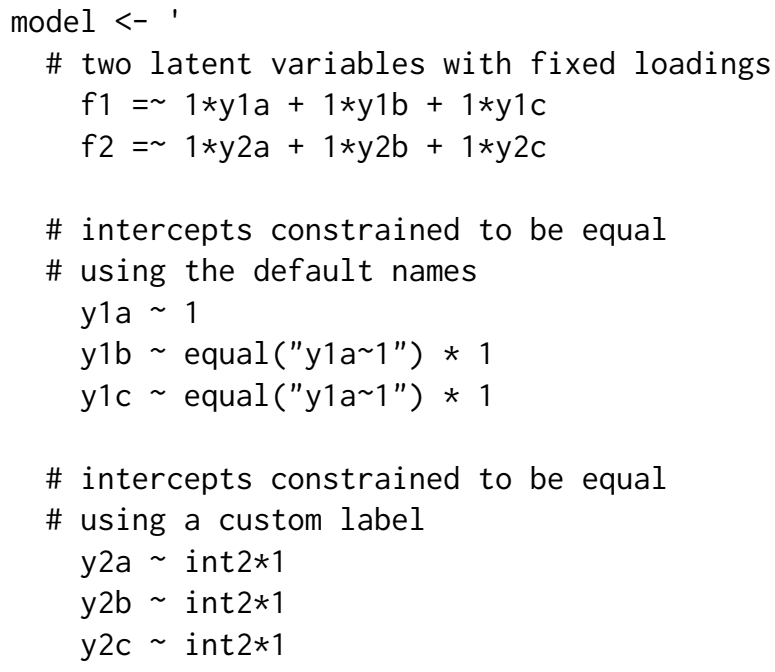

\section{Multiple groups}

In a multiple group analysis, modifiers that contain a single element should be replaced by a vector, having the same length as the number of groups. If you provide a single element, it will be recycled for all the groups. This may be dangerous, in particular when the modifier is a label. In that case, the (same) label is copied across all groups, and this would imply an equality constraint across groups. Therefore, when using modifiers in a multiple group setting, it is always safer (and cleaner) to specify the same number of elements as the number of groups. Consider this example with two groups:

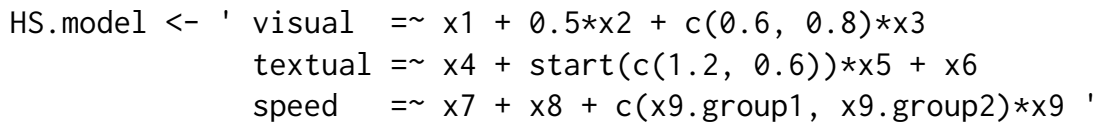

In this example, the factor loading of the ' $\mathrm{x} 2$ ' indicator is fixed to the value 0.5 for both groups. However, the factor loadings of the ' $\mathrm{x} 3$ ' indicator are fixed to 0.6 and 0.8 for group 1 and group 2 respectively. The same logic is used for all modifiers. Note that character vectors can contain unquoted strings.

\section{Multiple modifiers}

In the model syntax, you can specify a variable more than once on the right hand side of an operator; therefore, several 'modifiers' can be applied simultaneously; for example, if you want to fix the value of a parameter and also label that parameter, you can use something like:

$f 1=\sim x 1+x 2+4 * x 3+x 3$. loading $* x 3$ 


\section{References}

Yves Rosseel (2012). lavaan: An R Package for Structural Equation Modeling. Journal of Statistical Software, 48(2), 1-36. doi:10.18637/jss.v048.i02

modificationIndices Modification Indices

\section{Description}

Given a fitted lavaan object, compute the modification indices (= univariate score tests) for a selected set of fixed-to-zero parameters.

Usage

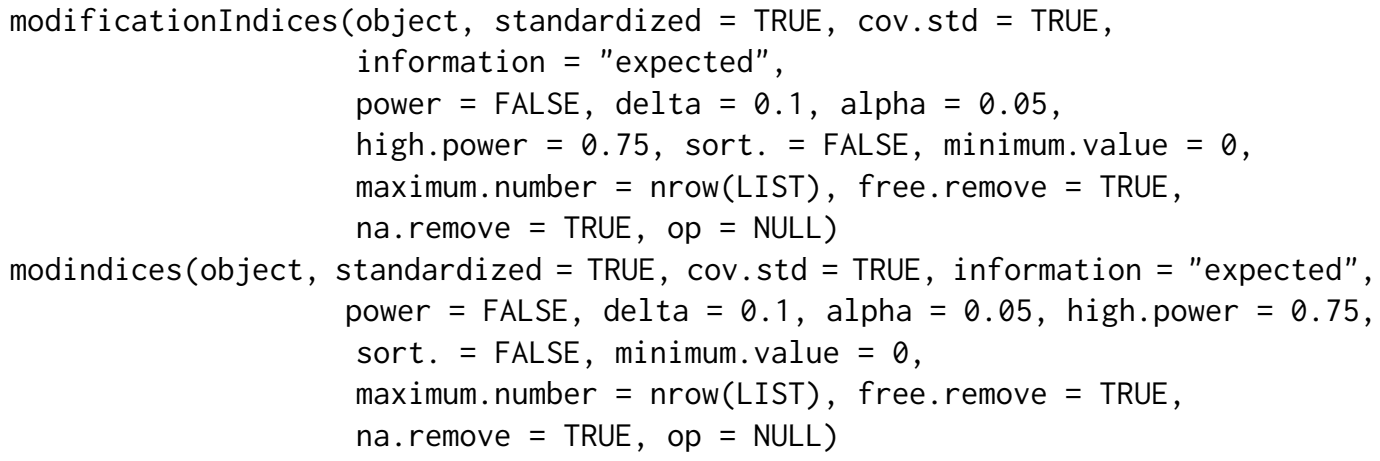

\section{Arguments}

object

standardized

cov.std

information

power

delta

alpha
An object of class lavaan.

If TRUE, two extra columns (sepc.lv and sepc.all) will contain standardized values for the EPCs. In the first column (sepc.lv), standardization is based on the variances of the (continuous) latent variables. In the second column (sepc.all), standardization is based on both the variances of both (continuous) observed and latent variables. (Residual) covariances are standardized using (residual) variances.

Logical. See standardizedSolution.

character indicating the type of information matrix to use (check lavInspect for available options). "expected" information is the default, which provides better control of Type I errors.

If TRUE, the (post-hoc) power is computed for each modification index, using the values of delta and alpha.

The value of the effect size, as used in the post-hoc power computation, currently using the unstandardized metric of the epc column.

The significance level used for deciding if the modification index is statistically significant or not. 
high.power If the computed power is higher than this cutoff value, the power is considered 'high'. If not, the power is considered 'low'. This affects the values in the 'decision' column in the output.

sort. Logical. If TRUE, sort the output using the values of the modification index values. Higher values appear first.

minimum.value Numeric. Filter output and only show rows with a modification index value equal or higher than this minimum value.

maximum. number Integer. Filter output and only show the first maximum number rows. Most useful when combined with the sort. option.

free.remove Logical. If TRUE, filter output by removing all rows corresponding to free (unconstrained) parameters in the original model.

na.remove Logical. If TRUE, filter output by removing all rows with NA values for the modification indices.

op

Character string. Filter the output by selecting only those rows with operator op.

\section{Details}

Modification indices are just 1-df (or univariate) score tests. The modification index (or score test) for a single parameter reflects (approximately) the improvement in model fit (in terms of the chisquare test statistic), if we would refit the model but allow this parameter to be free. This function is a convenience function in the sense that it produces a (hopefully sensible) table of currently fixed-tozero (of fixed to another constant) parameters. For each of these parameters, a modification index is computed, together with an expected parameter change (epc) value. It is important to realize that this function will only consider fixed-to-zero parameters. If you have equality constraints in the model, and you wish to examine what happens if you release all (or some) of these equality constraints, use the lavTestScore function.

\section{Value}

A data.frame containing modification indices and EPC's.

\section{Examples}

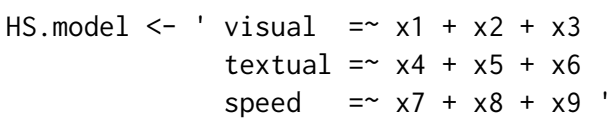

fit $<-$ cfa(HS.model, data=HolzingerSwineford1939)

modindices (fit, minimum. value $=10$, sort $=$ TRUE)

mplus2lavaan

mplus to lavaan converter

\section{Description}

Read in an Mplus input file, convert it to lavaan syntax, and fit the model. 


\section{Usage}

mplus2lavaan (inpfile, run = TRUE)

\section{Arguments}

inpfile The filename (including a full path) of the Mplus input file. The data (as referred to in the Mplus input file) should be in the same directory as the Mplus input file.

run Whether to run the specified Mplus input syntax (TRUE) or only to parse and convert the syntax (FALSE).

\section{Value}

A lavaan object with the fitted results of the Mplus model. The parsed and converted Mplus syntax is preserved in the eexternal slot of the lavaan object in the \$mplus . inp element. If run is FALSE, a list of converted syntax is returned.

\section{Author(s)}

Michael Hallquist

\section{See Also}

lavExport

\section{Examples}

\#\# Not run:

out <- mplus2lavaan("ex5.1.inp")

summary (out)

\#\# End(Not run)

mplus2lavaan. modelSyntax

Convert Mplus model syntax to lavaan

\section{Description}

Converts Mplus model syntax into lavaan model syntax.

\section{Usage}

mplus2lavaan.modelSyntax (syntax) 


\section{Arguments}

syntax

A character vector containing Mplus model syntax to be converted to lavaan model syntax. Note that parsing Mplus syntax often requires correct usage of newline characters. If syntax is a vector of multiple strings, these will be joined with newlines prior to conversion. Alternatively, \n characters can be included inline in syntax.

\section{Value}

A character string of converted lavaan model syntax.

\section{Author(s)}

Michael Hallquist

\section{See Also}

mplus2lavaan

\section{Examples}

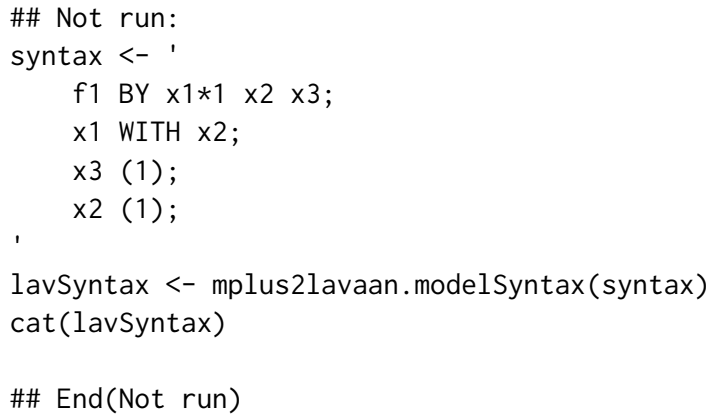

Parameter Estimates

\section{Description}

Parameter estimates of a latent variable model.

\section{Usage}

parameterEstimates (object

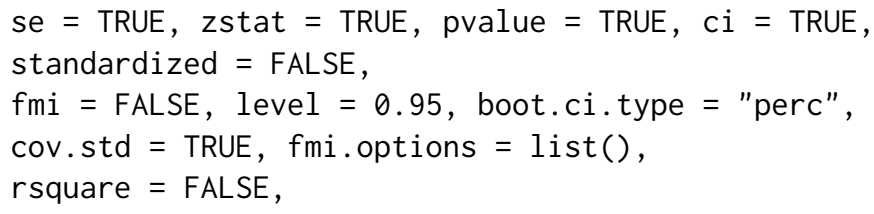




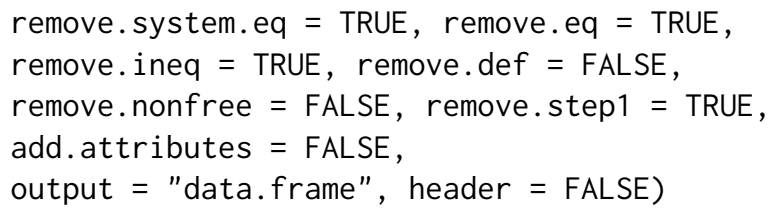

\section{Arguments}

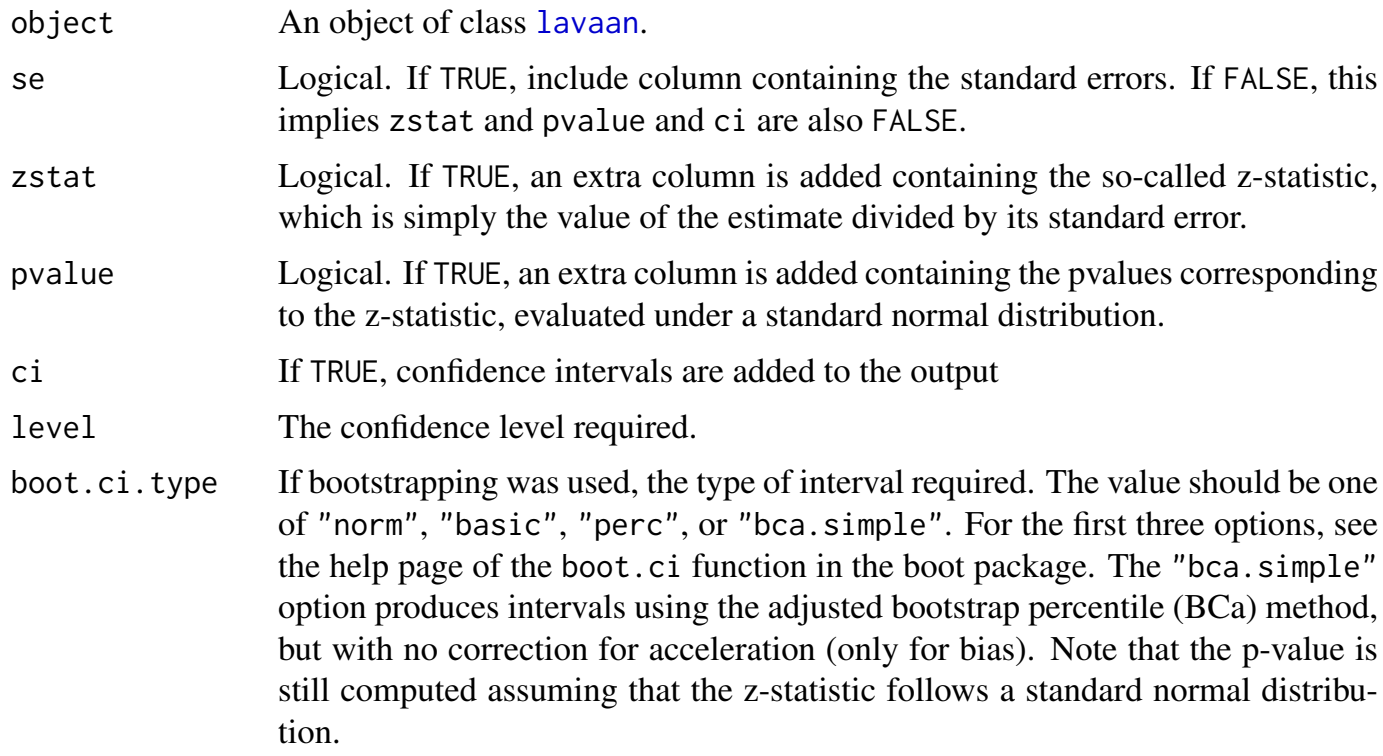

Logical. If TRUE, an extra column is added containing the so-called z-statistic, which is simply the value of the estimate divided by its standard error.

Logical. If TRUE, an extra column is added containing the pvalues corresponding to the z-statistic, evaluated under a standard normal distribution.

If TRUE, confidence intervals are added to the output

The confidence level required.

If bootstrapping was used, the type of interval required. The value should be one of "norm", "basic", "perc", or "bca.simple". For the first three options, see the help page of the boot.ci function in the boot package. The "bca.simple" option produces intervals using the adjusted bootstrap percentile (BCa) method, but with no correction for acceleration (only for bias). Note that the p-value is still computed assuming that the z-statistic follows a standard normal distribution.

fmi.options List. If non-empty, arguments can be provided to alter the default options when

standardized Logical. If TRUE, standardized estimates are added to the output. Note that $S E$ s and tests are still based on unstandardized estimates. Use standardizedSolution to obtain $S E$ s and test statistics for standardized estimates.

cov.std Logical. If TRUE, the (residual) observed covariances are scaled by the square root of the 'Theta' diagonal elements, and the (residual) latent covariances are scaled by the square root of the 'Psi' diagonal elements. If FALSE, the (residual) observed covariances are scaled by the square root of the diagonal elements of the observed model-implied covariance matrix (Sigma), and the (residual) latent covariances are scaled by the square root of diagonal elements of the modelimplied covariance matrix of the latent variables.

Logical. If TRUE, an extra column is added containing the fraction of missing information for each estimated parameter. Only available if estimator="ML", missing=" (fi $) \mathrm{ml} "$, and se="standard". See references for more information. the model is fitted with the complete(d) data; otherwise, the same options are used as the original model.

Logical. If TRUE, filter the output by removing all rows containing user-specified equality constraints, if any.

remove.system.eq

Logical. If TRUE, filter the output by removing all rows containing systemgenerated equality constraints, if any. 
remove.ineq

Logical. If TRUE, filter the output by removing all rows containing inequality constraints, if any.

remove.def Logical. If TRUE, filter the output by removing all rows containing parameter definitions, if any.

remove.nonfree Logical. If TRUE, filter the output by removing all rows containing fixed (nonfree) parameters.

remove.step1 Logical. Only used by sam(). If TRUE, filter the output by removing all rows corresponding to the measurement parameters that are part of the first step.

rsquare Logical. If TRUE, add additional rows containing the rsquare values (in the est column) of all endogenous variables in the model. Both the lhs and rhs column contain the name of the endogenous variable, while the codeop column contains $r 2$, to indicate that the values in the est column are rsquare values.

add.attributes Deprecated argument. Please use output= instead.

output Character. If "data.frame", the parameter table is displayed as a standard (albeit lavaan-formatted) data.frame. If "text" (or alias "pretty"), the parameter table is prettyfied, and displayed with subsections (as used by the summary function).

header Logical. Only used if output = "text". If TRUE, print a header at the top of the parameter list. This header contains information about the information matrix, if saturated (h1) model is structured or unstructured, and which type of standard errors are shown in the output.

\section{Value}

A data.frame containing the estimated parameters, parameters, standard errors, and (by default) zvalues, p-values, and the lower and upper values of the confidence intervals. If requested, extra columns are added with standardized versions of the parameter estimates.

\section{References}

Savalei, V. \& Rhemtulla, M. (2012). On obtaining estimates of the fraction of missing information from FIML. Structural Equation Modeling: A Multidisciplinary Journal, 19(3), 477-494.

\section{Examples}

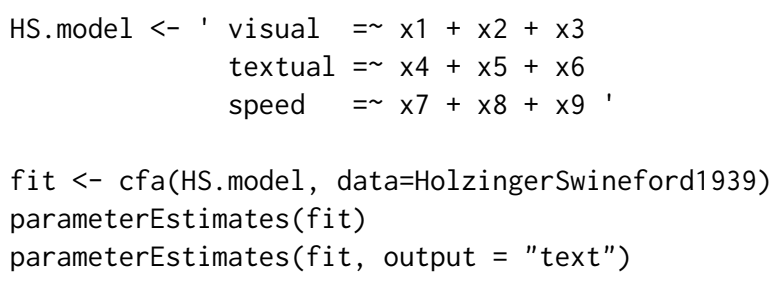


parTable Parameter Table

\section{Description}

Show the parameter table of a fitted model.

\section{Usage}

parameterTable(object)

parTable(object)

\section{Arguments}

object An object of class lavaan.

\section{Value}

A data. frame containing the model parameters. This is simply the output of the lavaanify function coerced to a data. frame (with stringsAsFactors $=$ FALSE).

\section{See Also}

lavaanify.

\section{Examples}

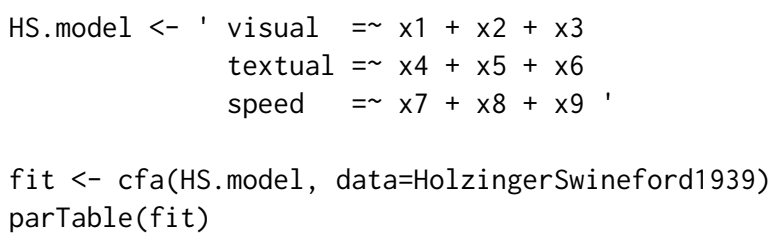

PoliticalDemocracy $\quad$ Industrialization And Political Democracy Dataset

\section{Description}

The 'famous' Industrialization and Political Democracy dataset. This dataset is used throughout Bollen's 1989 book (see pages 12, 17, 36 in chapter 2, pages 228 and following in chapter 7, pages 321 and following in chapter 8). The dataset contains various measures of political democracy and industrialization in developing countries.

\section{Usage}

data(PoliticalDemocracy) 


\section{Format}

A data frame of 75 observations of 11 variables.

y1 Expert ratings of the freedom of the press in 1960

y2 The freedom of political opposition in 1960

y3 The fairness of elections in 1960

y4 The effectiveness of the elected legislature in 1960

y5 Expert ratings of the freedom of the press in 1965

y6 The freedom of political opposition in 1965

y7 The fairness of elections in 1965

y8 The effectiveness of the elected legislature in 1965

x1 The gross national product (GNP) per capita in 1960

x2 The inanimate energy consumption per capita in 1960

x3 The percentage of the labor force in industry in 1960

\section{Source}

The dataset was originally retrieved from http://web. missouri .edu/ kolenikovs/Stat9370/democindus. txt (link no longer valid; see discussion on SEMNET 18 Jun 2009). The dataset is part of a larger (public) dataset (ICPSR 2532), see https://www. icpsr.umich.edu/web/ICPSR/studies/2532.

\section{References}

Bollen, K. A. (1989). Structural Equations with Latent Variables. Wiley Series in Probability and Mathematical Statistics. New York: Wiley.

Bollen, K. A. (1979). Political democracy and the timing of development. American Sociological Review, 44, 572-587.

Bollen, K. A. (1980). Issues in the comparative measurement of political democracy. American Sociological Review, 45, 370-390.

\section{Examples}

head(PoliticalDemocracy)

\section{Description}

Fit a Structural Equation Model (SEM) using the Structural After Measurement (SAM) approach. 


\section{Usage}

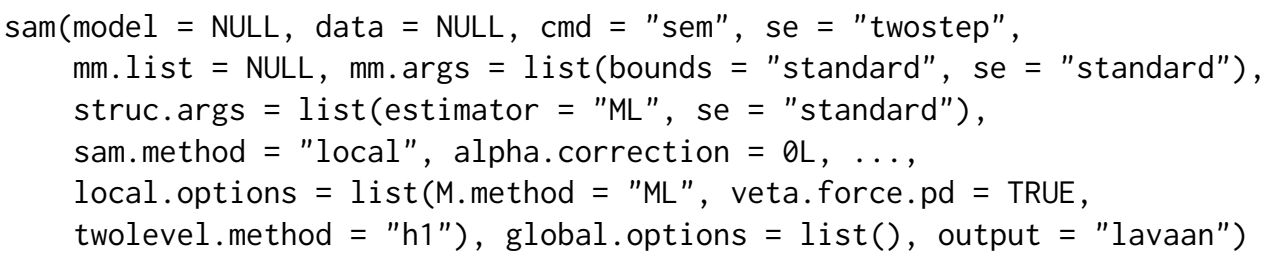

\section{Arguments}

model

A description of the user-specified model. Typically, the model is described using the lavaan model syntax. See model . syntax for more information. Alternatively, a parameter table (eg. the output of the lavaanify() function) is also accepted.

data A data frame containing the observed variables used in the model.

cmd Character. Which command is used to run the sem models. The possible choices are "sem", "cfa" or "lavaan", determining how we deal with default options.

se $\quad$ Character. The type of standard errors that are used in the final (structural) model. If "twostep" (the default), the standard errors take the estimation uncertainty of the first (measurement) stage into account. If "standard", this uncertainty is ignored, and we treat the measurement information as known. If "none", no standard errors are computed.

mm.list List. Define the measurement blocks. Each element of the list should be either a single name of a latent variable, or a vector of latent variable names. If omitted, a separate measurement block is used for each latent variable.

mm.args List. Optional arguments for the fitting function(s) of the measurement block(s) only. See lavOptions for a complete list.

struc.args List. Optional arguments for the fitting function of the structural part only. See lavOptions for a complete list.

sam.method Character. Can be set to "local", "global" or "fsr". In the latter case, the results are the same as if Bartlett factor scores were used, without any bias correction.

alpha.correction

Integer. Acceptable values are in the range 0 till N-1. If zero (the default), no small sample correction is performed, and the bias-correction is the same as with local SAM. When equal to $\mathrm{N}-1$, the bias-correction is eliminated, and the results are the same as naive FSR. Typical values are $0, P+1$ (where $\mathrm{P}$ is the number of predictors in the structural model), $\mathrm{P}+5$, and $(\mathrm{N}-1) / 2$.

... Many more additional options can be defined, using 'name = value'. See lavoptions for a complete list. These options affect both the measurement blocks and the structural part.

local.options List. Options specific for local SAM method (these options may change over time).

global.options List. Options specific for global SAM method (not used for now).

output Character. If "lavaan", a lavaan object returned. If "list", a list is returned with all the ingredients from the different stages. 


\section{Details}

The sam function tries to automate the SAM approach, by first estimating the measurement part of the model, and then the structural part of the model. See reference for more details.

\section{Value}

If output = "lavaan", an object of class lavaan, for which several methods are available, including a summary method. If output = "list", a list.

\section{References}

Rosseel and Loh (2021). A structural-after-measurement approach to Structural Equation Modeling. Psychological Methods. Advance online publication. https://dx.doi.org/10.1037/met0000503

\section{See Also}

lavaan

\section{Examples}

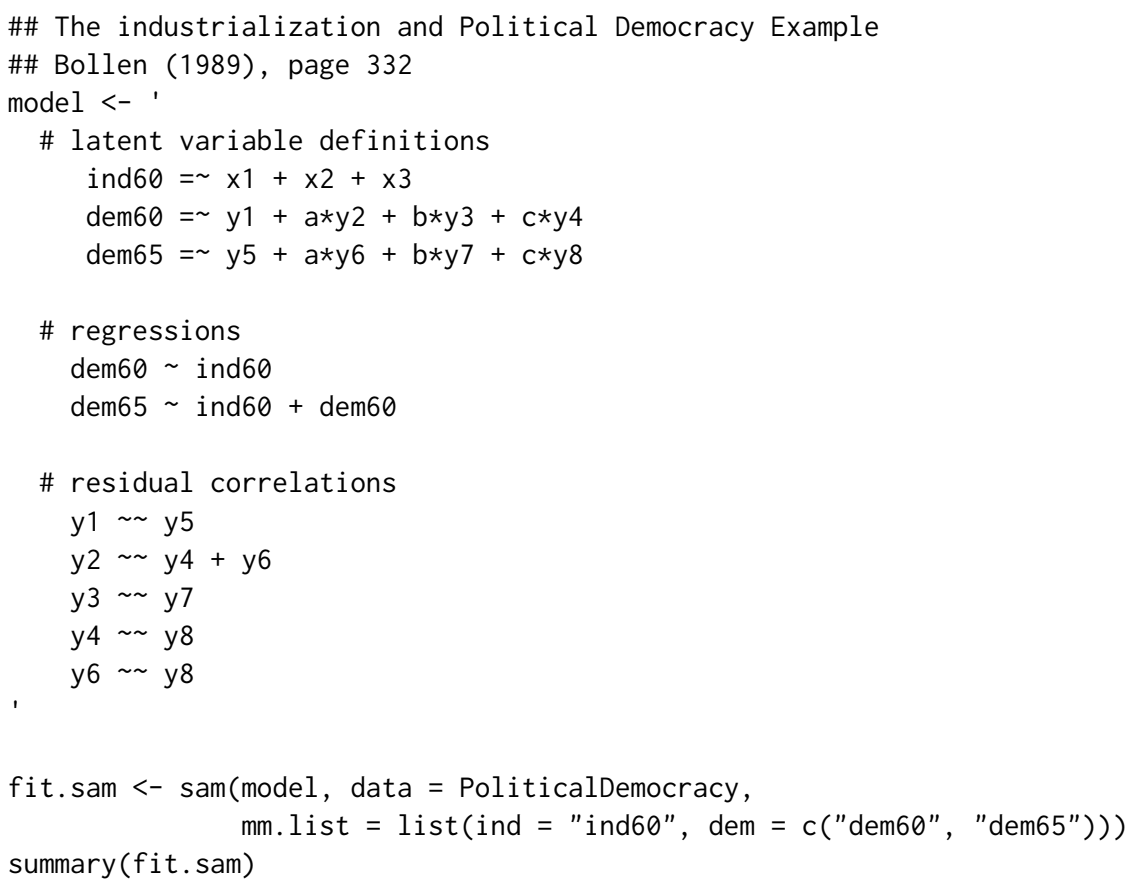




\section{Description}

Fit a Structural Equation Model (SEM).

\section{Usage}

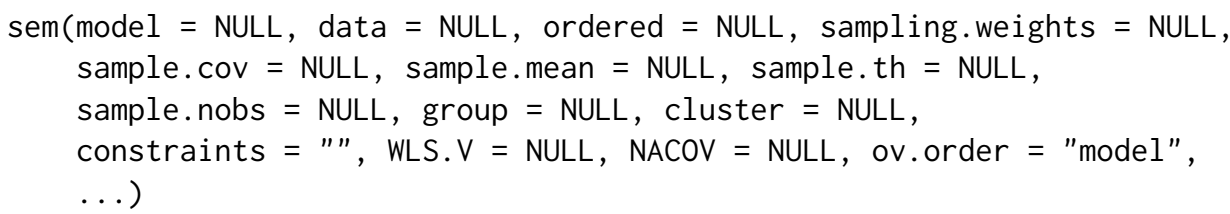

\section{Arguments}

model

data

ordered

sampling. weights

A variable name in the data frame containing sampling weight information. Currently only available for non-clustered data. Depending on the sampling. weights. normalization option, these weights may be rescaled (or not) so that their sum equals the number of observations (total or per group). Currently only available if estimator is ML in combination with robust standard errors and a robust test statistic. By default, the estimator will be "MLR".

sample.cov

sample.mean

sample.th

A description of the user-specified model. Typically, the model is described using the lavaan model syntax. See model. syntax for more information. Alternatively, a parameter table (eg. the output of the lavaanify() function) is also accepted.

An optional data frame containing the observed variables used in the model. If some variables are declared as ordered factors, lavaan will treat them as ordinal variables.

Character vector. Only used if the data is in a data.frame. Treat these variables as ordered (ordinal) variables, if they are endogenous in the model. Importantly, all other variables will be treated as numeric (unless they are declared as ordered in the data.frame.) Since 0.6-4, ordered can also be logical. If TRUE, all observed endogenous variables are treated as ordered (ordinal). If FALSE, all observed endogenous variables are considered to be numeric (again, unless they are declared as ordered in the data.frame.)

Numeric matrix. A sample variance-covariance matrix. The rownames and/or colnames must contain the observed variable names. For a multiple group analysis, a list with a variance-covariance matrix for each group.

A sample mean vector. For a multiple group analysis, a list with a mean vector for each group.

Vector of sample-based thresholds. For a multiple group analysis, a list with a vector of thresholds for each group. 
sample.nobs Number of observations if the full data frame is missing and only sample moments are given. For a multiple group analysis, a list or a vector with the number of observations for each group.

group Character. A variable name in the data frame defining the groups in a multiple group analysis.

cluster Character. A (single) variable name in the data frame defining the clusters in a two-level dataset.

constraints Additional (in)equality constraints not yet included in the model syntax. See model. syntax for more information.

WLS.V A user provided weight matrix to be used by estimator "WLS"; if the estimator is "DWLS", only the diagonal of this matrix will be used. For a multiple group analysis, a list with a weight matrix for each group. The elements of the weight matrix should be in the following order (if all data is continuous): first the means (if a meanstructure is involved), then the lower triangular elements of the covariance matrix including the diagonal, ordered column by column. In the categorical case: first the thresholds (including the means for continuous variables), then the slopes (if any), the variances of continuous variables (if any), and finally the lower triangular elements of the correlation/covariance matrix excluding the diagonal, ordered column by column.

NACOV A user provided matrix containing the elements of ( $\mathrm{N}$ times) the asymptotic variance-covariance matrix of the sample statistics. For a multiple group analysis, a list with an asymptotic variance-covariance matrix for each group. See the WLS.V argument for information about the order of the elements.

ov.order Character. If "model" (the default), the order of the observed variable names (as reflected for example in the output of lavNames () ) is determined by the model syntax. If "data", the order is determined by the data (either the full data.frame or the sample (co)variance matrix). If the WLS.V and/or NACOV matrices are provided, this argument is currently set to "data".

... Many more additional options can be defined, using 'name = value'. See lavoptions for a complete list.

\section{Details}

The sem function is a wrapper for the more general lavaan function, but setting the following default options: int.ov. free $=$ TRUE, int. lv. free $=F A L S E$, auto. fix. first $=$ TRUE (unless std. lv $=$ TRUE), auto. fix. single $=$ TRUE, auto.var $=$ TRUE, auto. cov.lv. $x=$ TRUE, auto. efa $=$ TRUE, auto. th $=$ TRUE, auto. delta $=$ TRUE, and auto. cov $. y=$ TRUE.

\section{Value}

An object of class lavaan, for which several methods are available, including a summary method.

\section{References}

Yves Rosseel (2012). lavaan: An R Package for Structural Equation Modeling. Journal of Statistical Software, 48(2), 1-36. doi:10.18637/jss.v048.i02 


\section{See Also}

lavaan

\section{Examples}

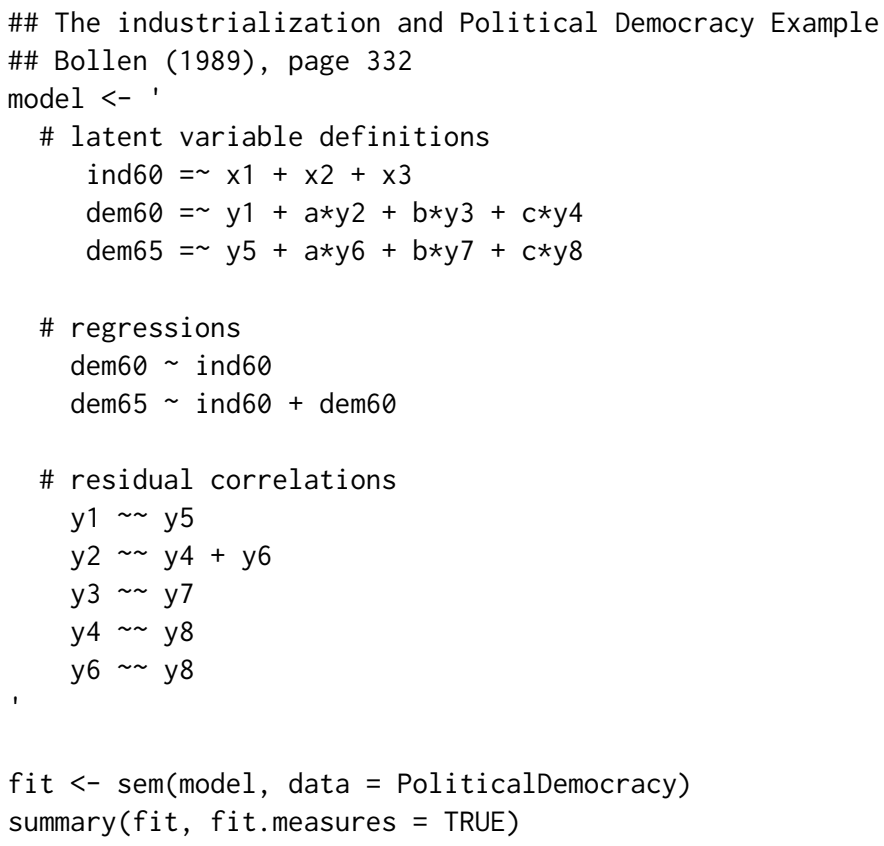

\section{Description}

Simulate data starting from a lavaan model syntax.

\section{Usage}

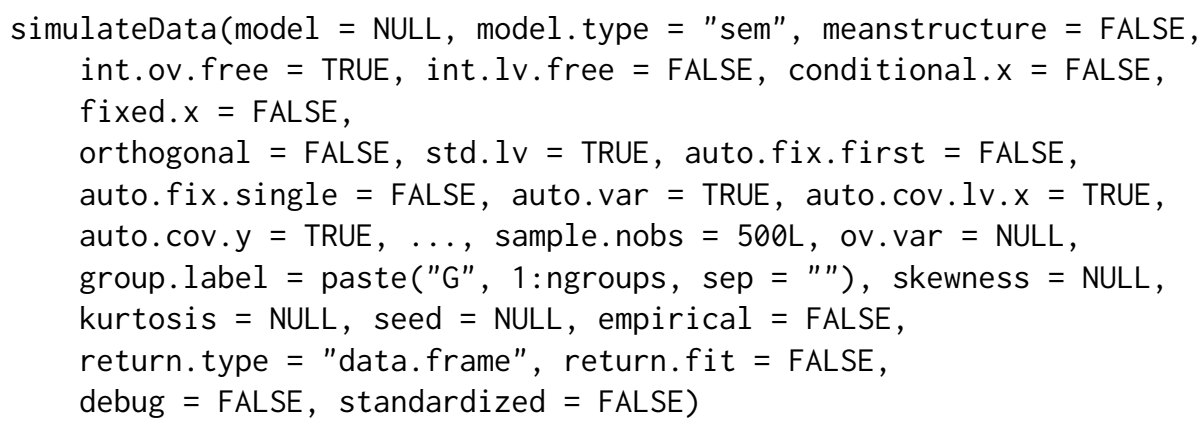




\section{Arguments}

model

model. type

meanstructure

int.ov.free

int.lv.free

conditional. $x$

fixed.x

or thogonal

std. lv
A description of the user-specified model. Typically, the model is described using the lavaan model syntax. See model syntax for more information. Alternatively, a parameter table (eg. the output of the lavaanify() function) is also accepted.

Set the model type: possible values are "cfa", "sem" or "growth". This may affect how starting values are computed, and may be used to alter the terminology used in the summary output, or the layout of path diagrams that are based on a fitted lavaan object.

If TRUE, the means of the observed variables enter the model. If "default", the value is set based on the user-specified model, and/or the values of other arguments.

If FALSE, the intercepts of the observed variables are fixed to zero.

If FALSE, the intercepts of the latent variables are fixed to zero.

If TRUE, we set up the model conditional on the exogenous ' $\mathrm{X}$ ' covariates; the model-implied sample statistics only include the non-X variables. If FALSE, the exogenous ' $x$ ' variables are modeled jointly with the other variables, and the model-implied statistics refect both sets of variables. If "default", the value is set depending on the estimator, and whether or not the model involves categorical endogenous variables.

If TRUE, the exogenous ' $x$ ' covariates are considered fixed variables and the means, variances and covariances of these variables are fixed to their sample values. If FALSE, they are considered random, and the means, variances and covariances are free parameters. If "default", the value is set depending on the mimic option.

If TRUE, the exogenous latent variables are assumed to be uncorrelated.

If TRUE, the metric of each latent variable is determined by fixing their variances to 1.0. If FALSE, the metric of each latent variable is determined by fixing the factor loading of the first indicator to 1.0.

auto.fix.first If TRUE, the factor loading of the first indicator is set to 1.0 for every latent variable.

auto.fix.single

If TRUE, the residual variance (if included) of an observed indicator is set to zero if it is the only indicator of a latent variable.

auto.var If TRUE, the residual variances and the variances of exogenous latent variables are included in the model and set free.

auto.cov.lv.x If TRUE, the covariances of exogenous latent variables are included in the model and set free.

auto.cov.y If TRUE, the covariances of dependent variables (both observed and latent) are included in the model and set free.

additional arguments passed to the lavaan function.

sample.nobs Number of observations. If a vector, multiple datasets are created. If return. type = "matrix" or return.type = "cov", a list of length (sample.nobs) is returned, with either the data or covariance matrices, each one based on the number of observations as specified in sample. nobs. If return. type = "data. frame", 
all datasets are merged and a group variable is added to mimic a multiple group dataset.

ov.var The user-specified variances of the observed variables.

group.label The group labels that should be used if multiple groups are created.

skewness Numeric vector. The skewness values for the observed variables. Defaults to zero.

kurtosis Numeric vector. The kurtosis values for the observed variables. Defaults to zero.

seed Set random seed.

empirical Logical. If TRUE, the implied moments (Mu and Sigma) specify the empirical not population mean and covariance matrix.

return.type If "data.frame", a data.frame is returned. If "matrix", a numeric matrix is returned (without any variable names). If "cov", a covariance matrix is returned (without any variable names).

return.fit If TRUE, return the fitted model that has been used to generate the data as an attribute (called "fit"); this may be useful for inspection.

debug If TRUE, debugging information is displayed.

standardized If TRUE, the residual variances of the observed variables are set in such a way such that the model implied variances are unity. This allows regression coefficients and factor loadings (involving observed variables) to be specified in a standardized metric.

\section{Details}

Model parameters can be specified by fixed values in the lavaan model syntax. If no fixed values are specified, the value zero will be assumed, except for factor loadings and variances, which are set to unity by default. By default, multivariate normal data are generated. However, by providing skewness and/or kurtosis values, nonnormal multivariate data can be generated, using the Vale \& Maurelli (1983) method.

\section{Value}

The generated data. Either as a data.frame (if return.type="data. frame"), a numeric matrix (if return.type="matrix"), or a covariance matrix (if return.type="cov").

\section{Examples}

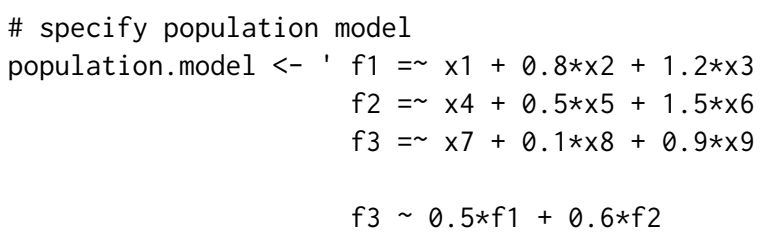




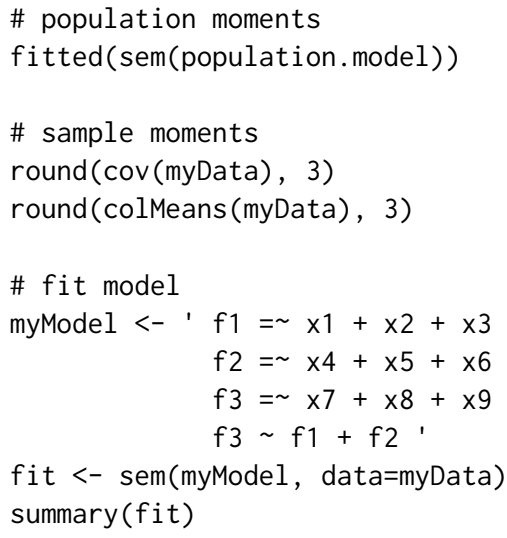

standardizedSolution Standardized Solution

\section{Description}

Standardized solution of a latent variable model.

\section{Usage}

standardizedSolution(object, type = "std.all", se = TRUE, zstat = TRUE, pvalue $=$ TRUE, ci $=$ TRUE, level $=0.95$, cov. std $=$ TRUE, remove. eq $=$ TRUE, remove. ineq $=$ TRUE, remove. def $=$ FALSE, partable $=$ NULL, GLIST $=$ NULL, est $=$ NULL, output = "data.frame")

\section{Arguments}

object

type

se

zstat

pvalue

ci

level
An object of class lavaan.

If "std. lv", the standardized estimates are on the variances of the (continuous) latent variables only. If "std. all", the standardized estimates are based on both the variances of both (continuous) observed and latent variables. If "std.nox", the standardized estimates are based on both the variances of both (continuous) observed and latent variables, but not the variances of exogenous covariates.

Logical. If TRUE, standard errors for the standardized parameters will be computed, together with a $\mathrm{z}$-statistic and a p-value.

Logical. If TRUE, an extra column is added containing the so-called z-statistic, which is simply the value of the estimate divided by its standard error.

Logical. If TRUE, an extra column is added containing the pvalues corresponding to the z-statistic, evaluated under a standard normal distribution.

If TRUE, simple symmetric confidence intervals are added to the output The confidence level required. 
cov.std

remove.eq

remove.ineq

remove.def

GLIST

est

partable

output
Logical. If TRUE, the (residual) observed covariances are scaled by the square root of the 'Theta' diagonal elements, and the (residual) latent covariances are scaled by the square root of the 'Psi' diagonal elements. If FALSE, the (residual) observed covariances are scaled by the square root of the diagonal elements of the observed model-implied covariance matrix (Sigma), and the (residual) latent covariances are scaled by the square root of diagonal elements of the modelimplied covariance matrix of the latent variables.

Logical. If TRUE, filter the output by removing all rows containing equality constraints, if any.

Logical. If TRUE, filter the output by removing all rows containing inequality constraints, if any.

Logical. If TRUE, filter the ouitput by removing all rows containing parameter definitions, if any.

List of model matrices. If provided, they will be used instead of the GLIST inside the object@Model slot. Only works if the est argument is also provided. See Note.

Numeric. Parameter values (as in the 'est' column of a parameter table). If provided, they will be used instead of the parameters that can be extract from object. Only works if the GLIST argument is also provided. See Note.

A custom list or data.frame in which to store the standardized parameter values. If provided, it will be used instead of the parameter table inside the object@ParTable slot.

Character. If "data. frame", the parameter table is displayed as a standard (albeit lavaan-formatted) data.frame. If "text" (or alias "pretty"), the parameter table is prettyfied, and displayed with subsections (as used by the summary function).

\section{Value}

A data.frame containing standardized model parameters.

\section{Note}

The est, GLIST, and partable arguments are not meant for everyday users, but for authors of external R packages that depend on lavaan. Only to be used with great caution.

\section{Examples}

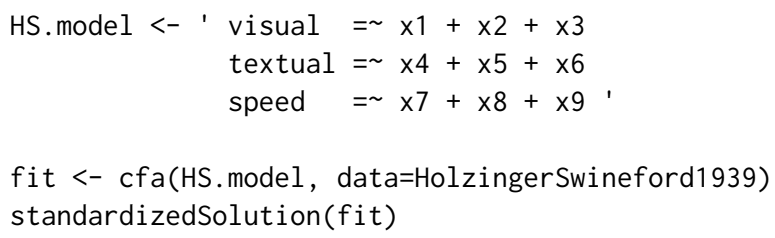




\section{Description}

S3 summary and print methods for class efaList.

\section{Usage}

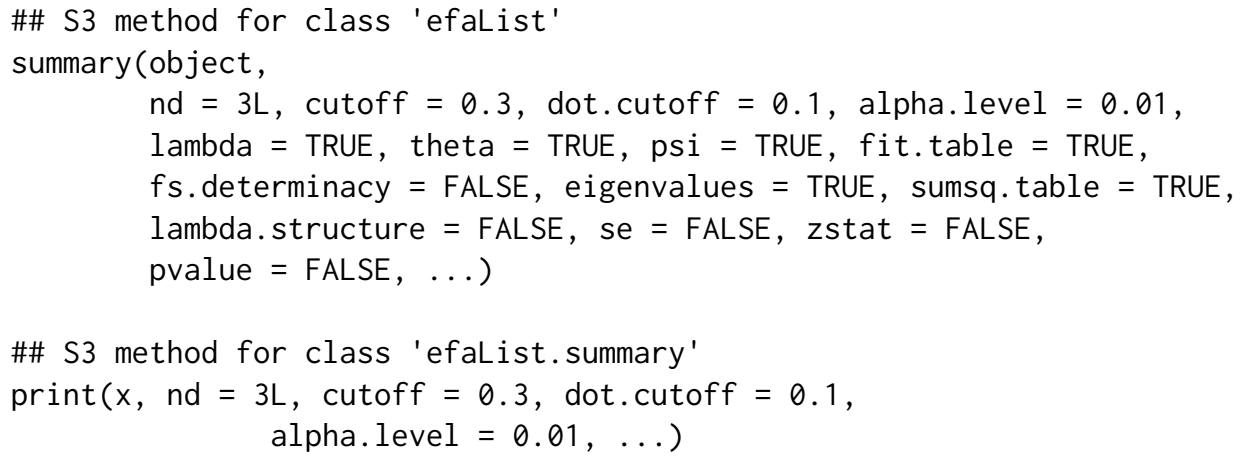

\section{Arguments}

object

$x$

nd

cutoff

dot.cutoff

alpha. level

lambda

theta

psi

fit. table
An object of class efaList, usually, a result of a call to efa with (the default) output $=$ "efa".

An object of class summary. efaList, usually, a result of a call to summary. efaList.

Integer. The number of digits that are printed after the decimal point in the output.

Numeric. Factor loadings smaller that this value (in absolute value) are not printed (even if they are significantly different from zero). The idea is that only medium to large factor loadings are printed, to better see the overall structure.

Numeric. Factor loadings larger (in absolute value) than this value, but smaller (in absolute value) than the cutoff value are shown as a dot. They represent small loadings that may still need your attention.

Numeric. If the the p-value of a factor loading is smaller than this value, a significance star is printed to the right of the factor loading. To switch this off, use alpha. level $=0$.

Logical. If TRUE, include the (standardized) factor loadings in the summary.

Logical. If TRUE, include the unique variances and the communalities in the table of factor loadings.

Logical. If TRUE, include the factor correlations in the summary. Ignored if only a single factor is used.

Logical. If TRUE, show fit information for each model. 
fs.determinacy Logical. If TRUE, show the factor score determinacy values per factor (assuming regression factor scores are used) and their squared values.

eigenvalues Logical. If TRUE, include the eigenvalues of the sample variance-covariance matrix in the summary.

sumsq. table Logical. If TRUE, include a table including sums of squares of factor loadings (and related measures) in the summary. The sums of squares are computed as the diagonal elements of Lambda times Psi (where Psi is the matrix of factor correlations.). If orthogonal rotation was used, Psi is diagonal and the sums of squares are identical to the sums of the squared column elements of the Lambda matrix (i.e., the factor loadings). This is no longer the case when obique rotation has been used. But in both cases (orthgonal or oblique), the (total) sum of the sums of squares equals the sum of the communalities. In the second row of the table (Proportion of total), the sums of squares are divided by the total. In the third row of the table (Proportion var), the sums of squares are divided by the number of items.

lambda.structure

Logical. If TRUE, show the structure matrix (i.e., the factor loadings multiplied by the factor correlations).

se Logical. If TRUE, include the standard errors of the standardized lambda, theta and psi elements in the summary.

zstat Logical. If TRUE, include the Z-statistics of the standardized lambda, theta and psi elements in the summary.

pvalue $\quad$ Logical. If TRUE, include the P-values of the standardized lambda, theta and psi elements in the summary.

... $\quad$ Further arguments passed to or from other methods.

\section{Value}

The function summary. efaList computes and returns a list of summary statistics for the list of EFA models in object.

\section{Examples}

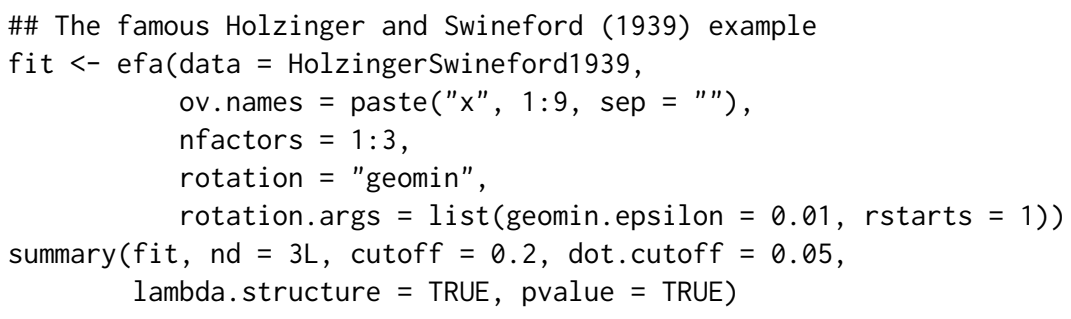


varTable Variable Table

\section{Description}

Summary information about the variables included in either a data.frame, or a fitted lavaan object.

\section{Usage}

varTable (object, ov.names $=$ names (object), ov.names.x $=$ NULL, ordered $=$ NULL, factor $=$ NULL, as.data. frame. $=$ TRUE)

\section{Arguments}

object

Either a data.frame, or an object of class lavaan.

ov. names

Only used if object is a data.frame. A character vector containing the variables that need to be summarized.

ov.names.x Only used if object is a data.frame. A character vector containing additional variables that need to be summarized.

ordered Character vector. Which variables should be treated as ordered factors

factor Character vector. Which variables should be treated as (unordered) factors?

as. data. frame. If TRUE, return the list as a data.frame.

\section{Value}

A list or data. frame containing summary information about variables in a data.frame. If object is a fitted lavaan object, it displays the summary information about the observed variables that are included in the model. The summary information includes variable type (numeric, ordered, ...), the number of non-missing values, the mean and variance for numeric variables, the number of levels of ordered variables, and the labels for ordered variables.

\section{Examples}

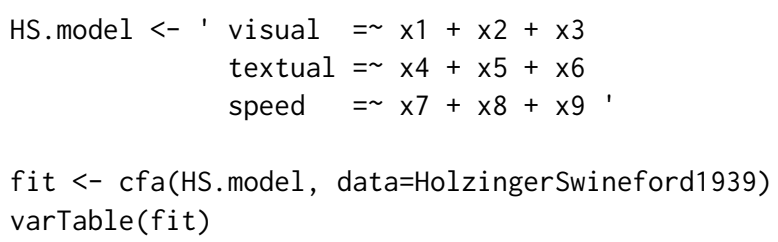




\section{Index}

AIC, 31

anova (lavTestLRT), 70

anova, lavaan-method (lavaan-class), 30

BIC, 31

bootstrapLavaan, 3

bootstrapLRT (bootstrapLavaan), 3

cfa, $5,20,29,30,32$

cfaList, 35,36

cfaList (lavaanList), 33

char2num (getCov), 15

coef, lavaan-method (lavaan-class), 30

coef, lavaanList-method

(lavaanList-class), 35

cor $2 \operatorname{cov}$ (getCov), 15

cov2cor, 16

Demo.growth, 8

Demo. twolevel, 9

efa, 10,111

efaList (summary.efaList), 111

estfun, 12

FacialBurns, 13

fitindices (fitMeasures), 13

fitMeasures, 13, 32

fitmeasures (fitMeasures), 13

fitMeasures, lavaan-method

(fitMeasures), 13

fitmeasures, lavaan-method

(fitMeasures), 13

fitted, lavaan-method (lavaan-class), 30

fitted.values, lavaan-method

(lavaan-class), 30

fsr (sam), 101

getCov, 15

growth, $8,16,29,30$ hist, 24

HolzingerSwineford1939, 19

InformativeTesting, 20

informativetesting

(InformativeTesting), 20

InformativeTesting methods, 23

inspect (lavInspect), 40

inspectSampleCov, 26

lav_constraints, 75

lav_constraints_parse

(lav_constraints), 75

lav_data, 76

lav_data_update (lav_data), 76

lav_func, 77

lav_func_gradient_complex (lav_func), 77

lav_func_gradient_simple (lav_func), 77

lav_func_jacobian_complex (lav_func), 77

lav_func_jacobian_simple (lav_func), 77

lav_matrix, 78

lav_matrix_antidiag_idx (lav_matrix), 78

lav_matrix_bdiag (lav_matrix), 78

lav_matrix_commutation (lav_matrix), 78

lav_matrix_commutation_mn_pre

(lav_matrix), 78

lav_matrix_commutation_pre

(lav_matrix), 78

lav_matrix_cov (lav_matrix), 78

lav_matrix_diag_idx (lav_matrix), 78

lav_matrix_diagh_idx (lav_matrix), 78

lav_matrix_duplication (lav_matrix), 78

lav_matrix_duplication_ginv

(lav_matrix), 78

lav_matrix_duplication_ginv_post

(lav_matrix), 78

lav_matrix_duplication_ginv_pre

(lav_matrix), 78

lav_matrix_duplication_ginv_pre_post

(lav_matrix), 78 
lav_matrix_duplication_post

(lav_matrix), 78

lav_matrix_duplication_pre

(lav_matrix), 78

lav_matrix_duplication_pre_post

(lav_matrix), 78

lav_matrix_lower2full (lav_matrix), 78

lav_matrix_orthogonal_complement (lav_matrix), 78

lav_matrix_symmetric_sqrt (lav_matrix), 78

lav_matrix_trace (lav_matrix), 78

lav_matrix_upper2full (lav_matrix), 78

lav_matrix_vec (lav_matrix), 78

lav_matrix_vech (lav_matrix), 78

lav_matrix_vech_col_idx (lav_matrix), 78

lav_matrix_vech_idx(lav_matrix), 78

lav_matrix_vech_reverse (lav_matrix), 78

lav_matrix_vech_row_idx (lav_matrix), 78

lav_matrix_vechr (lav_matrix), 78

lav_matrix_vechr_idx (lav_matrix), 78

lav_matrix_vechr_reverse (lav_matrix), 78

lav_matrix_vechru (lav_matrix), 78

lav_matrix_vechru_idx (lav_matrix), 78

lav_matrix_vechru_reverse (lav_matrix), 78

lav_matrix_vechu (lav_matrix), 78

lav_matrix_vechu_idx (lav_matrix), 78

lav_matrix_vechu_reverse (lav_matrix), 78

lav_matrix_vecr (lav_matrix), 78

lav_model, 82

lav_model_get_parameters (lav_model), 82

lav_model_implied (lav_model), 82

lav_model_set_parameters (lav_model), 82

lav_model_vcov_se (lav_model), 82

lav_partable, 83

lav_partable_add (lav_partable), 83

lav_partable_attributes (lav_partable), 83

lav_partable_complete (lav_partable), 83

lav_partable_constraints_ceq

(lav_constraints), 75

lav_partable_constraints_ciq

(lav_constraints), 75

lav_partable_constraints_def

(lav_constraints), 75 lav_partable_df (lav_partable), 83

lav_partable_from_lm (lav_partable), 83

lav_partable_independence

(lav_partable), 83

lav_partable_labels (lav_partable), 83

lav_partable_merge (lav_partable), 83

lav_partable_ndat (lav_partable), 83

lav_partable_npar (lav_partable), 83

lav_partable_unrestricted

(lav_partable), 83

lav_samplestats, 86

lav_samplestats_from_data

(lav_samplestats), 86

lavaan, 3, 4, 7, 11, 12, 14, 17, 18, 27, 29, 30, $34,36-40,46,50,57-59,61,63,65$, 67, 69, 70, 72, 74, 94, 98, 100, 103, 105-107, 109, 113

lavaan-class, 30

lavaanify, 39, 51, 100

lavaanify (model. syntax), 87

lavaanList, 33, 34-36, 47, 48

lavaanList-class, 35

lavaanNames (lavNames), 50

lavCor, 36

lavExport, 38, 96

lavImport (mplus2lavaan), 95

lavInspect, 27, 32, 40, 73, 94

lavListInspect, 46

lavListTech (lavListInspect), 46

lavLRT (lavTestLRT), 70

lavLRTTest (lavTestLRT), 70

lavMatrixRepresentation, 49

lavNames, 50

lavOptions, 7, 11, 18, 29, 37, 51, 102, 105

lavoptions (lav0ptions), 51

lavParseModelString (model.syntax), 87

lavParTable, 49, 84

lavParTable (model. syntax), 87

lavPartable, 49

lavPartable (model. syntax), 87

lavpartable (model.syntax), 87

lavPredict, 59, 62

lavpredict (lavPredict), 59

lavPredictY, 60, 61

lavResidual (lavResiduals), 63

lavResiduals, 63

lavScores (estfun), 12

lavScoreTest (lavTestScore), 72 
lavTables, 65, 69

lavTablesFit (lavTablesFitCp), 67

lavTablesFitCf, 71

lavTablesFitcf (lavTablesFitCp), 67

lavTablesFitCm (lavTablesFitCp), 67

lavTablesFitcp, 67

lavTech (lavInspect), 40

lavTest, 55, 69

lavtest (lavTest), 69

lavTestLRT, 31, 70

lavtestLRT (lavTestLRT), 70

lavTestScore, 31, 72, 95

lavtestscore (lavTestScore), 72

lavTestWald, 74

lavtestwald (lavTestWald), 74

lavWaldTest (lavTestWald), 74

logLik, lavaan-method (lavaan-class), 30

LRT (lavTestLRT), 70

model. syntax, 6, 7, 17, 18, 21, 27, 28, 33, 87, 102, 104, 105, 107

modificationIndices, 73, 94

modificationindices (modificationIndices), 94

modindices, 32

modindices (modificationIndices), 94

mplus2lavaan, 39, 95, 97

mplus2lavaan . modelSyntax, 96

nlminb, 56

nobs (lavaan-class), 30

nobs, lavaan-method (lavaan-class), 30

parameterEstimates, 32, 97

parameterestimates

(parameterEstimates), 97

parameterTable (parTable), 100

parametertable (parTable), 100

parseModelString (model.syntax), 87

parTable, 41, 47, 49, 51, 100

partable (parTable), 100

plot.InformativeTesting

(InformativeTesting methods), 23

PoliticalDemocracy, 100

predict, lavaan-method (lavaan-class), 30

print.efaList.summary

(summary.efaList), 111

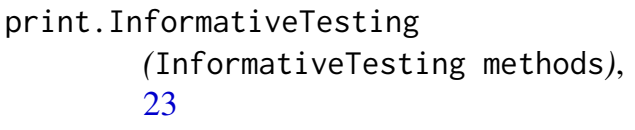

